\title{
ON THE RATE OF CONVERGENCE IN THE WEAK INVARIANCE PRINCIPLE FOR DEPENDENT RANDOM VARIABLES WITH APPLICATIONS TO MARKOV CHAINS
}

\author{
ION GRAMA*, EMILE LE PAGE^ AND MARC PEIGNÉ* \\ * UNIVERSITÉ DE BRETAGNE SUD, LMBA UMR CNRS 6205, VANNES, FRANCE \\ * UNIVERSITÉ F. RABELAIS TOURS, LMPT UMR CNRS 7350, TOURS, FRANCE
}

\begin{abstract}
We prove an invariance principle for non-stationary random processes and establish a rate of convergence under a new type of mixing condition. The dependence is exponentially decaying in the gap between the past and the future and is controlled by an assumption on the characteristic function of the finite dimensional increments of the process. The distinct feature of the new mixing condition is that the dependence increases exponentially in the dimension of the increments. The proposed mixing property is particularly suited for processes whose behavior can be described in terms of spectral properties of some related family of operators. Several examples are discussed. We also work out explicit expressions for the constants involved in the bounds. When applied to Markov chains our result specifies the dependence of the constants on the properties of the underlying Banach space and on the initial state of the chain.
\end{abstract}

\section{INTRODUCTION}

Let $\left(X_{k}\right)_{k \geq 1}$ be a sequence of real valued random variables (r.v.'s) defined on the probability space $(\Omega, \mathcal{F}, \mathbb{P})$ and $S_{N}(t)=N^{-1 / 2} \sum_{k=1}^{[N t]} X_{k}, t \in[0,1]$. The weak invariance principle for the process $\left(S_{N}(t)\right)_{0<t<1}$ is a powerful tool for various applications in probability and statistics. It extends the scope of the central limit theorem to continuous functionals of the stochastic process $\left(S_{N}(t)\right)_{0 \leq t \leq 1}$, such as, for example, the maxima or the $L^{2}$-norm of the trajectory of the process, considered in the appropriate functional spaces. The rates of convergence in the weak invariance principle, for independent r.v.'s have been obtained in Prokhorov [31], Borovkov [3], Komlós, Major and Tusnády [22], Einmahl [10], Sakhanenko [34], [35], Zaitsev [42] among others. For $\delta \leq \frac{1}{2}$, in the case of martingale-differences, the rates are essentially the same as in the independent case (see, for instance Hall and Heyde [19], Kubilius [23], Grama [11]). There are many recent results concerning the rates of convergence in the strong invariance principle for weakly dependent r.v.'s under various conditions. We refer to $\mathrm{Wu}$ [41], Zhao and Woodroofe [44], Liu and Lin [27], Cuny [5], Merlevède and Rio [28], Dedecker, Doukhan and Merlevède [7] and to the references therein. However, in contrast to independent r.v.'s where it is found that the optimal rate is of order $N^{-\frac{\delta}{3+2 \delta}}$, under the existence of the moments of order $2+2 \delta$, with $\delta>0$, the problem of obtaining

Date: February 5, 2013.

2000 Mathematics Subject Classification. Primary 60F17, 60J05, 60J10. Secondary 37C30 .

Key words and phrases. Rate of convergence, invariance principle, Markov chains, mixing, spectral gap. 
the best rate of convergence in the weak invariance principle for dependent variables is not yet settled completely.

Gouëzel [15] has introduced a new type of mixing condition which is tied to spectral properties of the sequence $\left(X_{k}\right)_{k \geq 1}$. Consider the vectors $\bar{X}_{1}=\left(X_{J_{1}}, \ldots, X_{J_{M_{1}}}\right)$ and $\bar{X}_{2}=$ $\left(X_{k_{g a p}+J_{M_{1}+1}}, \ldots, X_{k_{g a p}+J_{M_{1}+M_{2}}}\right)$, called the past and the future, respectively, where $X_{k+J_{m}}=$ $\sum_{l \in J_{m}} X_{k+l}, J_{m}=\left[j_{m-1}, j_{m}\right), j_{0} \leq \ldots \leq j_{M_{1}+M_{2}}$, and $k_{g a p}$ is a gap between $\bar{X}_{1}$ and $\bar{X}_{2}$. Roughly speaking, the condition used in [15] suppose that the characteristic function of $\left(\bar{X}_{1}, \bar{X}_{2}\right)$ is exponentially closed to the product of the characteristic functions of the past $\bar{X}_{1}$ and the future $\bar{X}_{2}$, with an error term of the form $A \exp \left(-\lambda k_{g a p}\right)$, where $\lambda$ is some non negative constant and $A$ is polynomial in terms of the size of the blocks. This mixing property is particularly suited for systems whose behavior can be described in terms of spectral properties of some related family of operators, as initiated by Nagaev [29], [30] and Guivarc'h [16]. Examples are Markov chains, whose perturbed transition probability operators $\left(\mathbf{P}_{t}\right)_{|t| \leq \varepsilon_{0}}$ exhibit a spectral gap and enough regularity in $t$, and dynamical systems, whose characteristic functions can be coded by a family of operators $\left(\mathcal{L}_{t}\right)_{|t| \leq \varepsilon_{0}}$ satisfying similar properties. Gouëzel proves in [15] an almost sure invariance principle with a rate of convergence. The proof relies on a progressive blocking technique (see Bernstein [2]) coupled with a triadic Cantor like scheme and on the Komlós, Major and Tusnády approximation type results for independent r.v.'s (see [22], [10], [42]), which is in contrast to approaches usually based on martingale methods.

The scope of the present paper is to obtain a rate of convergence in the weak invariance principle for dependent r.v.'s under the mixing condition introduced above. Although the strong and weak invariance principles are closely related, it seems that the rate of convergence in the weak invariance principle is less studied under weak dependence constraints. We refer to Doukhan, Leon and Portal [6], Merlevède and Rio [28] and Grama and Neumann [14]. However, these results rely on mixing conditions which are not verified in the present setting. Under the above mentioned mixing and some further mild conditions including the moment assumption $\sup _{k \geq 1} \mathbb{E}\left|X_{k}\right|^{2+2 \delta}<\infty$ we obtain a bound of the order $N^{-\frac{1+\alpha}{1+2 \alpha} \frac{\alpha}{3+2 \alpha}}$, for any $\alpha<\delta$. Moreover, we give explicit expressions of some constants involved in the rate of convergence; for instance, in the case of Markov walks we are able to figure out the dependence of the rate of convergence on the properties of the Banach space related to the corresponding family of perturbed transition operators $\left(\mathbf{P}_{t}\right)_{|t| \leq \varepsilon_{0}}$ and on the initial state $X_{0}=x$ of the associated Markov chain. When compared with the rate $N^{-\frac{1}{2} \frac{\alpha}{1+2 \alpha}}$ in the almost sure invariance principle of [15] ours appears with a loss in the power of multiple $\frac{2+2 \alpha}{3+2 \alpha}<1$. This loss in the power is exactly the same as in the case of independent r.v.'s, when we compare the almost sure invariance principle (rate $N^{-\frac{\delta}{2+2 \delta}}$ ) and the (weak) invariance principle (rate $N^{-\frac{\delta}{3+2 \delta}}$ ).

As a potential application of the obtained results we point out the asymptotic equivalence of statistical experiments as developed in [12], [13], [14], whose scope can be extended to various models under weak dependence constraints.

Our paper is organized as follows. In Sections 2 and 3 we formulate our main results and give an application to the case of Markov chains. In Section 4 we introduce the notations to be used in the proofs of the main results. Proofs of the main results are given in Sections 5 , 
6 and 9. In Section 7 we collect some auxiliary assertions and general facts and, finally, in Section 8 we prove some bounds for the $L^{p}$ norm of the increments of the process $\left(X_{k}\right)_{k>1}$.

We conclude this section by setting some notations to be used all over the paper. For any $x \in \mathbb{R}^{d}$, denote by $\|x\|_{\infty}=\sup _{1 \leq i \leq d}\left|x_{i}\right|$ the supremum norm. For any $p>0$, the $L^{p}$ norm of a random variable $X$ is denoted by $\|X\|_{L^{p}}$. In the sequel $\mathcal{L}\left(\left(Z_{i}^{\prime}\right)_{i>1} \mid \mathbb{P}^{\prime}\right) \stackrel{d}{=}$ $\mathcal{L}\left(\left(Z_{i}^{\prime \prime}\right)_{i \geq 1} \mid \mathbb{P}^{\prime \prime}\right)$ means the equality in distribution of two stochastic processes $\left(Z_{i}^{\prime}\right)_{i \geq 1}$ and $\left(Z_{i}^{\prime \prime}\right)_{i>1}$ possibly defined on two different probability spaces $\left(\Omega^{\prime}, \mathcal{F}^{\prime}, \mathbb{P}^{\prime}\right)$ and $\left(\Omega^{\prime \prime}, \mathcal{F}^{\prime \prime}, \mathbb{P}^{\prime \prime}\right)$. By $c, c^{\prime}, c^{\prime \prime}, \ldots$ supplied with indices $1,2, \ldots$ we denote absolute constants whose values may vary from line to line. The notations $c_{\alpha_{1}, \ldots, \alpha_{r}}, c_{\alpha_{1}, \ldots, \alpha_{r}}^{\prime}, \ldots$ will be used to stress that the constants depend only on the parameters indicated in their indices: for instance $c_{\alpha, \beta}^{\prime}$ denotes a constant depending only on the constants $\alpha, \beta$. All other constants will be specifically indicated. As usual, we shall use the shortcut "standard normal r.v." for a normal random variable of mean 0 and variance 1 .

\section{MAin RESUlts}

Assume that on the probability space $(\Omega, \mathcal{F}, \mathbb{P})$ we are given a sequence $\left(X_{i}\right)_{i>1}$ of dependent r.v.'s with values in the real line $\mathbb{R}$. The expectation with respect to $\mathbb{P}$ is denoted by $\mathbb{E}$.

The following condition will be used to ensure that the process $\left(X_{i}\right)_{i \geq 1}$ has almost independent increments. Given natural numbers $k_{\text {gap }}, M_{1}, M_{2} \in \mathbb{N}$ and a sequence $j_{0} \leq \ldots \leq j_{M_{1}+M_{2}}$ denote $X_{k+J_{m}}=\sum_{l \in J_{m}} X_{k+l}$, where $J_{m}=\left[j_{m-1}, j_{m}\right), m=1, \ldots, M_{1}+M_{2}$ and $k \geq 0$. Consider the vectors $\bar{X}_{1}=\left(X_{J_{1}}, \ldots, X_{J_{M_{1}}}\right)$ and $\bar{X}_{2}=\left(X_{k_{g a p}+J_{M_{1}+1}}, \ldots, X_{k_{g a p}+J_{M_{1}+M_{2}}}\right)$. Let $\phi\left(t_{1}, t_{2}\right)=\mathbb{E} e^{i t_{1} \bar{X}_{1}+i t_{2} \bar{X}_{2}}, \phi_{1}\left(t_{1}\right)=\mathbb{E} e^{i t_{1} \bar{X}_{1}}$ and $\phi_{2}\left(t_{2}\right)=\mathbb{E} e^{i t_{2} \bar{X}_{2}}$ be the characteristic functions of $\left(\bar{X}_{1}, \bar{X}_{2}\right), \bar{X}_{1}$ and $\bar{X}_{2}$ respectively. We ask that the dependence between the two vectors $\bar{X}_{1}$ (the past) and $\bar{X}_{2}$ (the future) decreases exponentially as a function of the size of the gap $k_{\text {gap }}$ in the following sense.

Condition C1 There exist positive constants $\varepsilon_{0} \leq 1, \lambda_{0}, \lambda_{1}, \lambda_{2}$ such that for any $k_{\text {gap }}, M_{1}, M_{2} \in$ $N$, any sequence $j_{0}<\ldots<j_{M_{1}+M_{2}}$ and any $t_{1} \in R^{M_{1}}, t_{2} \in R^{M_{2}}$ satisfying $\left\|\left(t_{1}, t_{2}\right)\right\|_{\infty} \leq \varepsilon_{0}$,

$$
\left|\phi\left(t_{1}, t_{2}\right)-\phi_{1}\left(t_{1}\right) \phi_{2}\left(t_{2}\right)\right| \leq \lambda_{0} \exp \left(-\lambda_{1} k_{g a p}\right)\left(1+\max _{m=1, \ldots, M_{1}+M_{2}} \operatorname{card}\left(J_{m}\right)\right)^{\lambda_{2}\left(M_{1}+M_{2}\right)} .
$$

All over the paper we suppose that the following moment conditions hold true.

Condition C2 There exist two constants $\delta>0$ and $\mu_{\delta}>0$ such that

$$
\sup _{i \geq 1}\left\|X_{i}\right\|_{L^{2+2 \delta}} \leq \mu_{\delta}<\infty
$$

We suppose also that the sequence $\left(X_{i}\right)_{i \geq 1}$ possesses the following asymptotic homogeneity property. 
Condition C3 There exist a constant $\tau>0$ and a positive number $\sigma>0$ such that, for any $\gamma>0$ and any $n \geq 1$,

$$
\sup _{k \geq 0}\left|n^{-1} \operatorname{Var}_{\mathbb{P}}\left(\sum_{i=k+1}^{k+n} X_{i}\right)-\sigma^{2}\right| \leq \tau n^{-1+\gamma}
$$

The main result of the paper is the following theorem. Denote $\mu_{i}=\mathbb{E} X_{i}$, for $i \geq 1$.

Theorem 1. Assume Conditions $\boldsymbol{C 1}, \boldsymbol{C 2}$ and $\boldsymbol{C 3}$. Let $0<\alpha<\delta$. Then, on some probability space $(\widetilde{\Omega}, \widetilde{\mathcal{F}}, \widetilde{\mathbb{P}})$ there exist a sequence of independent standard normal random variables $\left(W_{i}\right)_{i \geq 1}$ and a sequence of random variables $\left(\tilde{X}_{i}\right)_{i \geq 1}$ such that $\mathcal{L}\left(\left(\tilde{X}_{i}\right)_{i \geq 1} \mid \widetilde{\mathbb{P}}\right) \stackrel{d}{=} \mathcal{L}\left(\left(X_{i}\right)_{i \geq 1} \mid \mathbb{P}\right)$ and for any $0<\rho<\frac{\alpha}{2(1+2 \alpha)}$ and $N \geq 1$,

$$
\widetilde{\mathbb{P}}\left(N^{-1 / 2} \sup _{k \leq N}\left|\sum_{i=1}^{k}\left(\widetilde{X}_{i}-\mu_{i}-\sigma W_{i}\right)\right|>6 N^{-\rho}\right) \leq C_{0} N^{-\alpha \frac{1+\alpha}{1+2 \alpha}+\rho(2+2 \alpha)},
$$

where $C_{0}=c_{\lambda_{1}, \lambda_{2}, \alpha, \delta, \sigma}\left(1+\lambda_{0}+\mu_{\delta}+\sqrt{\tau}\right)^{2+2 \delta}$ and $c_{\lambda_{1}, \lambda_{2}, \alpha, \delta, \sigma}$ depends only on the constants indicated in its indices.

Letting $\rho=\frac{\alpha}{3+2 \alpha} \frac{1+\alpha}{1+2 \alpha}$ from Theorem 1 we get

$$
\widetilde{\mathbb{P}}\left(N^{-1 / 2} \sup _{k \leq N}\left|\sum_{i=1}^{k}\left(\widetilde{X}_{i}-\mu_{i}-\sigma W_{i}\right)\right|>6 N^{-\frac{\alpha}{3+2 \alpha} \frac{1+\alpha}{1+2 \alpha}}\right) \leq C_{0} N^{-\frac{\alpha}{3+2 \alpha} \frac{1+\alpha}{1+2 \alpha}}
$$

where $C_{0}=c_{\lambda_{1}, \lambda_{2}, \alpha, \delta, \sigma}\left(1+\lambda_{0}+\mu_{\delta}+\sqrt{\tau}\right)^{2+2 \delta}$ and $c_{\lambda_{1}, \lambda_{2}, \alpha, \delta, \sigma}$ depends only on the constants indicated in its indices. Compared with the optimal rate of convergence for independent r.v.'s $N^{-\frac{\alpha}{3+2 \alpha}}$ the loss in the power is within the factor $\frac{1+\alpha}{1+2 \alpha}$. As $\alpha \rightarrow \infty$ we obtain the limiting power $\frac{1}{4}$ which is twice worse than the optimal power $\frac{1}{2}$ in the independent case. In particular, if $\alpha=\frac{1}{2}$ (which corresponds to $p=2+2 \alpha=3$ ) we obtain the rate of convergence $N^{-\frac{\alpha}{3+2 \alpha} \frac{1+\alpha}{1+2 \alpha}}=N^{-\frac{3}{32}}$, while in the independent case we have $N^{-\frac{1}{8}}$, which represents a loss of the power of the order $\frac{1}{8}-\frac{3}{32}=\frac{1}{32}$.

We would like to point out that in Theorem 1 we figure out the explicit dependence of the constant $C_{0}$ on the constants $\lambda_{0}, \mu_{\delta}$ and $\tau$ involved in Conditions C1, C2 and C3. In the next section we show that Theorem 1 can be applied to Markov walks under spectral gap type assumptions on the associate Markov chain. It is important to stress that our result is the first one to figure out the dependence of the constants involved in the bounds on the initial state of the Markov chain. The results of the paper can be also applied to a large class of dynamical systems, however this stays beyond the scope of the present paper. For a discussion of these type of applications we refer to [15].

For the proof of Theorem 1, without loss of generality, we shall assume that $\mu_{i}=0$, $i \geq 1$ and $\sigma=1$, since the general case case can be reduced to this one by centering and renormalising the variables $X_{i}$, i.e. by replacing $X_{i}$ by $X_{i}^{\prime}=\left(X_{i}-\mu_{i}\right) / \sigma$. It is easy to see that Conditions $\mathbf{C 1}, \mathbf{C 2}, \mathbf{C} 3$ are satisfied for the new random variables $X_{i}^{\prime}$ with the same $\lambda_{0}$ and with $\mu_{\delta}, \tau$ replaced by $2 \mu_{\delta} / \sigma, \tau / \sigma^{2}$. 


\section{Applications to Markov walks}

Consider a Markov chain $\left(X_{k}\right)_{k \geq 0}$ with values in the measurable state space $(\mathbb{X}, \mathcal{X})$ with the transition probability $\mathbf{P}(x, \cdot), x \in \mathbb{X}$. For every $x \in \mathbb{X}$ denote by $\mathbb{P}_{x}$ and $\mathbb{E}_{x}$ the probability measure and expectation generated by the finite dimensional distributions

$$
\mathbb{P}_{x}\left(X_{0} \in B_{0}, \ldots, X_{n} \in B_{n}\right)=1_{B_{0}}(x) \int_{B_{1}} \ldots \int_{B_{n}} \mathbf{P}\left(x, d x_{1}\right) \ldots \mathbf{P}\left(x_{n-1}, d x_{n}\right),
$$

for any $B_{k} \in \mathcal{X}, k=1, \ldots, n, n=1,2, \ldots$ on the space of trajectories $(\mathbb{X}, \mathcal{X})^{\mathbb{N}}$. In particular $\mathbb{P}_{x}\left(X_{0}=x\right)=1$.

Let $f$ be a real valued function defined on the state space $\mathbb{X}$ of the Markov chain $\left(X_{k}\right)_{k>0}$. Let $\mathcal{B}$ be a Banach space of real valued functions on $\mathbb{X}$ endowed with the norm $\|\cdot\|_{\mathcal{B}}$ and let $\|\cdot\|_{\mathcal{B} \rightarrow \mathcal{B}}$ be the operator norm on $\mathcal{B}$. Denote by $\mathcal{B}^{\prime}=\mathcal{L}(\mathcal{B}, \mathbb{C})$ the topological dual of $\mathcal{B}$. The unit function on $\mathbb{X}$ is denoted by $e: e(x)=1$ for $x \in \mathbb{X}$. The Dirac measure at $x \in \mathbb{X}$ is denoted by $\boldsymbol{\delta}_{x}: \boldsymbol{\delta}_{x}(g)=g(x)$ for any $g \in \mathcal{B}$ and is seen as a linear functional on $\mathcal{B}$.

Introduce the following hypotheses.

\section{Hypothesis M1 (Banach space):}

a) The unit function e belongs to $B$.

b) For every $x \in X$ the Dirac measure $\boldsymbol{\delta}_{x}$ belongs to $B^{\prime}$.

c) $B \subseteq L^{1}(\mathbf{P}(x, \cdot))$ for every $x \in X$.

d) There exists a constant $\varepsilon_{0} \in(0,1)$ such that for any $g \in B$ the function $e^{i t f} g \in B$ for any $t$ satisfying $|t| \leq \varepsilon_{0}$.

Note that, for any $x \in \mathbb{X}$ and $g \in L^{1}(\mathbf{P}(x, \cdot))$, the quantity $\mathbf{P} g(x):=\int_{\mathbb{X}} g(y) \mathbf{P}(x, d y)$ is well defined. In particular, under the hypothesis $\mathbf{M} 1 \mathrm{c}), \mathbf{P} g(x)$ exists when $g \in \mathcal{B}$. We thus consider the following hypothesis:

\section{Hypothesis M2 (Spectral gap):}

a) The map $g \mapsto \mathbf{P} g$ is a bounded operator on $B$

b) There exist constants $C_{Q}>0$ and $\kappa \in(0,1)$ such that

$$
\mathbf{P}=\Pi+Q
$$

where $\Pi$ is a one dimensional projector and $Q$ is an operator on $B$ satisfying $\Pi Q=Q \Pi=0$ and $\left\|Q^{n}\right\|_{\mathcal{B} \rightarrow \mathcal{B}} \leq C_{Q} \kappa^{n}$.

Notice that, since the image of $\Pi$ is generated by the unit function $e$, there exists a linear form $\nu \in \mathcal{B}^{\prime}$ such that for any $g \in \mathcal{B}$,

$$
\Pi g=\nu(g) e .
$$

When hypotheses $\mathbf{M} 1$ and $\mathbf{M} 2$ hold, we set $\mathbf{P}_{t} g=\mathbf{P}\left(e^{i t f} g\right)$ for any $g \in \mathcal{B}$ and $t \in\left[-\varepsilon_{0}, \varepsilon_{0}\right]$. Notice that $\mathbf{P}=\mathbf{P}_{0}$.

\section{Hypothesis M3 (Perturbed transition operator):}

a) For any $|t| \leq \varepsilon_{0}$ the map $g \in B \rightarrow P_{t} g \in B$ is a bounded operator on $B$,

b) There exists a constant $C_{\mathbf{P}}>0$ such that, for all $n \geq 1$ and $|t| \leq \varepsilon_{0}$,

$$
\left\|\mathbf{P}_{t}^{n}\right\|_{\mathcal{B} \rightarrow \mathcal{B}} \leq C_{\mathbf{P}}
$$


Hypothesis M4 (Moment conditions): There exists $\delta>0$ such that for any $x \in X$,

$$
\mu_{\delta}(x):=\sup _{k \geq 1}\left(\mathbb{E}_{x}\left|f\left(X_{k}\right)\right|^{2+2 \delta}\right)^{\frac{1}{2+2 \delta}}=\sup _{k \geq 1}\left(\left(\mathbf{P}^{k}|f|^{2+2 \delta}\right)(x)\right)^{\frac{1}{2+2 \delta}}<\infty .
$$

We show first that under the hypotheses M1, M2, M3 and M4, conditions C1, C2 and C3 are satisfied. As in the previous section let $k_{\text {gap }}, M_{1}, M_{2} \in \mathbb{N}$ and $j_{0} \leq \ldots \leq$ $j_{M_{1}+M_{2}}$ be natural numbers. Denote $Y_{k+J m}=\sum_{l \in J_{m}} f\left(X_{k+l}\right)$, where $J_{m}=\left[j_{m-1}, j_{m}\right)$, $m=1, \ldots, M_{1}+M_{2}$ and $k \geq 0$. Consider the vectors $\bar{Y}_{1}=\left(Y_{J_{1}}, \ldots, Y_{J_{M_{1}}}\right)$ and $\bar{Y}_{2}=$ $\left(Y_{k_{\text {gap }}+J_{M_{1}+1}}, \ldots, Y_{k_{\text {gap }}+J_{M_{1}+M_{2}}}\right)$. Let $\phi_{x}\left(t_{1}, t_{2}\right)=\mathbb{E} e^{i t_{1} \bar{Y}_{1}+i t_{2} \bar{Y}_{2}}, \phi_{x, 1}\left(t_{1}\right)=\mathbb{E}_{x} e^{i t_{1} \bar{Y}_{1}}$ and $\phi_{x, 2}\left(t_{2}\right)=$ $\mathbb{E}_{x} e^{i t_{2} \bar{Y}_{2}}$ be the characteristic functions of $\left(\bar{Y}_{1}, \bar{Y}_{2}\right), \bar{Y}_{1}$ and $\bar{Y}_{2}$ respectively.

Proposition 1. Assume that the Markov chain $\left(X_{n}\right)_{n \geq 1}$ and the function $f$ satisfy the hypotheses $\mathbf{M 1}, \mathbf{M} 2, \mathbf{M} 3$ and $\mathbf{M}$. Then Condition $\overline{\boldsymbol{C}} \mathbf{1}$ is satisfied, i.e. there exists a positive constant $\varepsilon_{0} \leq 1$ such that for any $k_{\text {gap }}, M_{1}, M_{2} \in \mathbb{N}$, any sequence $j_{0}<\ldots<j_{M_{1}+M_{2}}$ and any $t_{1} \in \mathbb{R}^{M_{1}}, t_{2} \in \mathbb{R}^{M_{2}}$ satisfying $\left\|\left(t_{1}, t_{2}\right)\right\|_{\infty} \leq \varepsilon_{0}$,

$\left|\phi_{x}\left(t_{1}, t_{2}\right)-\phi_{x, 1}\left(t_{1}\right) \phi_{x, 2}\left(t_{2}\right)\right| \leq \lambda_{0}(x) \exp \left(-\lambda_{1} k_{g a p}\right)\left(1+\max _{m=1, \ldots, M_{1}+M_{2}} \operatorname{card}\left(J_{m}\right)\right)^{\lambda_{2}\left(M_{1}+M_{2}\right)}$,

where $\lambda_{0}(x)=2 C_{Q}\left(\|\nu\|_{\mathcal{B}^{\prime}}+\left\|\boldsymbol{\delta}_{x}\right\|_{\mathcal{B}^{\prime}}\right)\|e\|_{\mathcal{B}}, \lambda_{1}=|\ln \kappa|$ and $\lambda_{2}=\max \left\{1, \log _{2} C_{\mathbf{P}}\right\}$.

Proposition 2. Assume that the Markov chain $\left(X_{n}\right)_{n \geq 1}$ and the function $f$ satisfy the hypotheses $\mathbf{M 1}, \mathbf{M 2}, \mathbf{M 3}$ and $\mathbf{M 4}$. Then:

a) There exists a constant $\mu$ such that for any $x \in \mathbb{X}$ and $k \geq 1$

$$
\left|\mathbb{E}_{x} f\left(X_{k}\right)-\mu\right| \leq A_{1}(x) \kappa^{k \gamma / 4-1},
$$

for any positive constant $\gamma$ satisfying $0<\gamma \leq \min \{1,2 \delta\}$, where $A_{1}(x)=c\left\|\boldsymbol{\delta}_{x}\right\|_{\mathcal{B}^{\prime}}\|e\|_{\mathcal{B}} C_{\mathbf{P}} C_{Q}+$ $c_{\delta} \mu_{\delta}(x)^{1+\gamma}$. Moreover

$$
\sum_{k=0}^{\infty}\left|\mathbb{E}_{x} f\left(X_{k}\right)-\mu\right| \leq \bar{\mu}(x)=c_{\delta, \kappa, \gamma} A_{1}(x)
$$

for a constant $c_{\delta, \kappa, \gamma}$ depending only on its indices.

b) There exists a constant $\sigma \geq 0$ such that for any $x \in \mathbb{X}$,

$$
\sup _{m \geq 0}\left|\operatorname{Var}_{\mathbb{P}_{x}}\left(\sum_{i=m+1}^{m+n} f\left(X_{i}\right)\right)-n \sigma^{2}\right| \leq \tau(x)=c_{\delta, \kappa, \gamma} A_{2}(x),
$$

where

$$
A_{2}(x)=1+\mu_{\delta}(x)^{2+\gamma}+\left(1+\left\|\boldsymbol{\delta}_{x}\right\|_{\mathcal{B}^{\prime}}\right)\|e\|_{\mathcal{B}}\left(C_{\mathbf{P}}^{2} C_{Q}\left(1+C_{Q}\right)+C_{\mathbf{P}} C_{Q}\left(1+\|\nu\|_{\mathcal{B}^{\prime}} C_{\mathbf{P}}\right)\right) .
$$

Note that the constants $\mu$ and $\sigma$ do not depend on the initial state $x$.

The main result of this section is the following theorem. Let $\widetilde{\Omega}=\mathbb{R}^{\infty} \times \mathbb{R}^{\infty}$. For any $\widetilde{\omega}=\left(\widetilde{\omega}_{1}, \widetilde{\omega}_{2}\right) \in \widetilde{\Omega}$ denote by $\widetilde{Y}_{i}=\widetilde{\omega}_{1, i}$ and $W_{i}=\widetilde{\omega}_{2, i}, i \geq 1$, the coordinate processes in $\widetilde{\Omega}$. 
Theorem 2. Assume that the Markov chain $\left(X_{n}\right)_{n \geq 0}$ and the function $f$ satisfy the hypotheses M1, M2, M3 and M4. Let $0<\alpha<\delta$. Assume that $\sigma>0$. Then there exists a Markov transition kernel $x \rightarrow \widetilde{\mathbb{P}}_{x}(\cdot)$ from $(\mathbb{X}, \mathcal{X})$ to $(\widetilde{\Omega}, \mathcal{B}(\widetilde{\Omega}))$ such that $\mathcal{L}\left(\left(\widetilde{Y}_{i}\right)_{i \geq 1} \mid \widetilde{\mathbb{P}}_{x}\right) \stackrel{d}{=}$ $\mathcal{L}\left(\left(f\left(X_{i}\right)\right)_{i>1} \mid \mathbb{P}_{x}\right),\left(W_{i}\right)_{i \geq 1}$ are independent standard normal r.v.'s under $\widetilde{\mathbb{P}}_{x}$ and for any $0<\rho<\frac{\alpha}{2(1+2 \alpha)}$

$$
\widetilde{\mathbb{P}}_{x}\left(N^{-\frac{1}{2}} \sup _{k \leq N}\left|\sum_{i=1}^{k}\left(\widetilde{Y}_{i}-\mu-\sigma W_{i}\right)\right|>6 N^{-\rho}\right) \leq C(x) N^{-\alpha \frac{1+\alpha}{1+2 \alpha}+\rho(2+2 \alpha)},
$$

with

$$
C(x)=C_{1}\left(1+\mu_{\delta}(x)+\left\|\boldsymbol{\delta}_{x}\right\|_{\mathcal{B}^{\prime}}\right)^{2+2 \delta},
$$

where $C_{1}$ is a constant depending only on $\delta, \alpha, \kappa, C_{\mathbf{P}}, C_{Q},\|e\|_{\mathcal{B}}$ and $\|\nu\|_{\mathcal{B}^{\prime}}$.

Note that only the probability $\widetilde{\mathbb{P}}_{x}$ depends on the initial state $x$ while the processes $\left(\widetilde{Y}_{k}\right)_{k \geq 0}$ and $\left(W_{k}\right)_{k \geq 0}$ do not depend on it.

As in the previous section, letting $\rho=\frac{\alpha}{3+2 \alpha} \frac{1+\alpha}{1+2 \alpha}$, under the conditions of Theorem 2 we obtain,

$$
\widetilde{\mathbb{P}}_{x}\left(N^{-\frac{1}{2}} \sup _{k \leq N}\left|\sum_{i=1}^{k}\left(\widetilde{Y}_{i}-\mu-\sigma W_{i}\right)\right|>6 N^{-\frac{\alpha}{3+2 \alpha} \frac{1+\alpha}{1+2 \alpha}}\right) \leq C(x) N^{-\frac{\alpha}{3+2 \alpha} \frac{1+\alpha}{1+2 \alpha}}
$$

Compared to the rate $N^{-\frac{\alpha}{3+2 \alpha}}$ which is optimal in the independent case the rate of convergence $N^{-\frac{\alpha}{3+2 \alpha} \frac{1+\alpha}{1+2 \alpha}}$ in (3.8) is slower by the factor $N^{\frac{\alpha}{3+2 \alpha} \frac{\alpha}{1+2 \alpha}}$. As $\alpha \rightarrow \infty$ we obtain $N^{-\frac{1}{4}}$ which is the best rate in the invariance principle that is known for dependent random variables.

In Theorem 2 we do not suppose the existence of the stationary measure. Assume that there exists a stationary probability measure $\nu$ on $\mathbb{X}$; it thus coincides with the the linear form $\nu$ introduced in (3.2). Let $\mathbb{P}_{\nu}$ and $\mathbb{E}_{\nu}$ the probability measure and expectation generated by the finite dimensional distributions of the chain under the stationary measure $\nu$. Note that, the means $\mathbb{E}_{\nu} X_{k}$ and the covariances $\operatorname{Cov}_{\mathbb{P}_{\nu}}\left(f\left(X_{l}\right), f\left(X_{l+k}\right)\right)$ with respect to $\nu$ may not exist, under the hypotheses M1, M2, M3 and M4. To ensure their existence, we require the following additional condition (where generally $|f|^{2} \notin \mathcal{B}$ ).

Hypothesis M5 (Stationary measure): On the state space $\mathbb{X}$ there exists a stationary probability measure $\nu$ such that $\nu\left(\sup _{k \geq 0} \mathbf{P}^{k}\left(|f|^{2}\right)\right)<\infty$.

Under Hypothesis M5 for $\mu$ and $\sigma$ we find the usual expressions of the means and of the variance in the central limit theorem for dependent r.v.'s.

Theorem 3. Assume that the Markov chain $\left(X_{n}\right)_{n \geq 0}$ and the function $f$ satisfy the hypotheses M1, M2, M3, M4 and M5. Assume also that $\sigma_{\nu}>0$. Then Proposition 2 holds true with $\mu=\nu(f)$ and $\sigma=\sigma_{\nu}$, where

$$
\nu(f)=\int f(x) \nu(d x)
$$


and

$$
\sigma_{\nu}^{2}=\operatorname{Var}_{\mathbb{P}_{\nu}}\left(f\left(X_{0}\right)\right)+2 \sum_{k=1}^{\infty} \operatorname{Cov}_{\mathbb{P}_{\nu}}\left(f\left(X_{0}\right), f\left(X_{k}\right)\right) .
$$

Moreover, if $\sigma_{\nu}>0$ the assertions of Theorem 2 and (3.8) hold true with $\mu=\nu(f)$ and $\sigma=\sigma_{\nu}$

From Theorem 3 one can derive a bound when the Markov chain $\left(X_{n}\right)_{n \geq 0}$ is in the stationary regime. If we assume that $\nu\left(\sup _{k \geq 0} \mathbf{P}^{k}\left(|f|^{2+2 \delta}\right)\right) \leq c_{\nu, \delta}<\infty$ and $\int\left\|\boldsymbol{\delta}_{x}\right\|_{\mathcal{B}^{\prime}}^{2+2 \delta} \nu(d x) \leq$ $c_{\mathcal{B}^{\prime}, \delta}<\infty$, then integrating (3.7) with respect to $\nu$ we obtain

$$
\widetilde{\mathbb{P}}_{\nu}\left(N^{-\frac{1}{2}} \sup _{k \leq N}\left|\sum_{i=1}^{k}\left(\widetilde{Y}_{i}-\mu-\sigma W_{i}\right)\right|>6 N^{-\rho}\right) \leq C N^{-\alpha \frac{1+\alpha}{1+2 \alpha}+\rho(2+2 \alpha)},
$$

where $C$ is a constant depending on $\delta, \alpha, \kappa, C_{\mathbf{P}}, C_{Q},\|e\|_{\mathcal{B}},\|\nu\|_{\mathcal{B}^{\prime}}$ and $c_{\nu, \delta}, c_{\mathcal{B}^{\prime}, \delta}$.

The hypotheses M1, M2, M4 and M5 formulated above can be easily verified by standard methods. As to M3 it can be verified using two approaches. The first approach is based on the assumption that the family of operators $\left(\mathbf{P}_{t}\right)_{|t| \leq \varepsilon_{0}}$ is continuous in $t$ at $t=0$. In this case, M3 is satisfied by classical perturbation theory (see, for instance Dunford and Schwartz $[9])$. The second approach is based on a weaker form of continuity of the family $\left(\mathbf{P}_{t}\right)_{|t| \leq \varepsilon_{0}}$ as developed in Keller and Liverani [21].

We end this section by giving three examples where these hypotheses are satisfied.

3.1. Example 1 (Markov chains with finite state spaces). Suppose that $\left(X_{n}\right)_{n \geq 0}$ is an irreducible ergodic aperiodic Markov chain with finite state space. It is easy to verify that the hypotheses M1, M2, M3, M4 and M5 are satisfied and that there exists a unique invariant probability measure $\nu$. Then the conclusions of Theorem 3 hold true.

3.2. Example 2 (autoregressive random walk with Bernoulli noise). Consider the autoregressive model $x_{n+1}=\alpha x_{n}+b_{n}, n \geq 0$, where $\alpha$ is a constant satisfying $\alpha \in(-1,1)$ and $\left(b_{n}\right)_{n \geq 0}$ are i.i.d. Bernoulli r.v.'s with $P(b=1)=P(b=-1)=\frac{1}{2}$ and $x_{0}=x$. Consider the Banach space $\mathcal{B}=\mathcal{L}$ of continuous functions $f$ on $\mathbb{R}$ such that $\|f\|=|f|+[f]<\infty$, where

$$
|f|=\sup _{x \in \mathbb{R}} \frac{|f(x)|}{1+x^{2}}, \quad[f]=\sup _{\substack{x, y \in \mathbb{R} \\ x \neq y}} \frac{|f(x)-f(y)|}{|x-y|\left(1+x^{2}\right)\left(1+y^{2}\right)} .
$$

Since $\alpha \in(-1,1)$, the invariant measure $\nu$ exists and coincides with the law of the random variable $Z=\sum_{i=1}^{\infty} \alpha^{i-1} b_{i}$. It is easy to verify that hypotheses M1, M2, M3, M4 and M5 are satisfied for the function $f(x)=x$. For the the mean $\nu(f)$ we have

$$
\nu(f)=\int x \nu(d x)=\mathbb{E} Z=\sum_{i=1}^{\infty} \alpha^{i-1} \mathbb{E} b_{1}=\frac{\mathbb{E} b_{1}}{1-\alpha} .
$$

Since $\mathbb{E} b_{1}=0$, one gets $\nu(f)=0$ and the variance is computed as follows:

$$
\sigma_{\nu}^{2}=\lim _{n \rightarrow \infty} \mathbb{E}\left(\sum_{i=1}^{n} \alpha^{i-1} b_{i}\right)^{2}=\lim _{n \rightarrow \infty} \sum_{i=1}^{n} \alpha^{2(i-1)} \mathbb{E} b_{1}^{2}=\frac{1}{1-\alpha^{2}} .
$$


Thus the conclusions of Theorem 3 hold true with $\nu(f)=0$ and $\sigma_{\nu}^{2}=\frac{1}{1-\alpha^{2}}$.

3.3. Example 3 (stochastic recursion). On the probability space $(\Omega, \mathcal{F}, \mathbb{P})$ consider the stochastic recursion

$$
x_{n+1}=a_{n+1} x_{n}+b_{n+1}, n \geq 0,
$$

where $\left(a_{n}, b_{n}\right)_{n \geq 0}$ are i.i.d. r.v.'s with values in $(0, \infty) \times \mathbb{R}$ of the same distribution $\widehat{\mu}$ and $x_{0}=x$. Following Guivarc'h and Le Page [17], we assume the conditions:

H1 There exists $\alpha>2$ such that $\varphi(\alpha):=\int|a|^{\alpha} \widehat{\mu}(d a, d b)<1$ and $\int|b|^{\alpha} \widehat{\mu}(d a, d b)<+\infty$.

H2 $\widehat{\mu}\left(\left\{(a, b): a x_{0}+b=x_{0}\right\}\right)<1$ for any $x_{0} \in R$.

H3 The set $\{\ln |a|:(a, b) \in \operatorname{supp} \widehat{\mu}\}$ generates a dense subgroup of $R$.

Let $\varepsilon \in(0,1), \theta$ and $c$ be positive such that $\alpha-1<c+\varepsilon<\theta \leq 2 c<\alpha-\varepsilon$. Consider the Banach space $\mathcal{B}=\mathcal{L}_{\varepsilon, c, \theta}$ of continuous functions $f$ on $\mathbb{R}$ such that $\|f\|=|f|+[f]<\infty$, where

$$
|f|=\sup _{x \in \mathbb{R}} \frac{|f(x)|}{1+|x|^{\theta}}, \quad[f]=\sup _{\substack{x, y \in \mathbb{R} \\ x \neq y}} \frac{|f(x)-f(y)|}{|x-y|^{\varepsilon}\left(1+|x|^{c}\right)\left(1+|y|^{c}\right)} .
$$

The transition probability $\mathbf{P}(x, \cdot)$ of the Markov chain $\left(x_{n}\right)_{n \geq 0}$ is defined by

$$
\int h(y) \mathbf{P}(x, d y)=\int h(a x+b) \widehat{\mu}(d a, d b),
$$

for any bounded Borel measurable function $h: \mathbb{R} \rightarrow \mathbb{R}$ and $x \in \mathbb{R}$. For any $x \in \mathbb{R}$ denote by $\mathbb{P}_{x}$ and $\mathbb{E}_{x}$ the corresponding probability measure and expectation generated by the finite dimensional distributions on the space of trajectories. It is proved in [17] (Proposition 1) that the series $\sum_{i=1}^{\infty} a_{1} \ldots a_{i-1} b_{i}$ is $\mathbb{P}$-a.s. convergent and the Markov chain $\left(x_{n}\right)_{n>0}$ has a unique invariant probability measure $\nu$ which coincides with the law of $Z=\sum_{i=1}^{\infty} a_{1} \ldots a_{i-1} b_{i}$. Moreover, it holds $\int|x|^{t} \nu(d x)<\infty$ for any $t \in[0, \alpha)$.

We shall verify that hypotheses M1, M2, M3, M4 and M5 are satisfied with the function $f(x)=x$. Hypothesis M1 is obvious and hypotheses M2 and M3 follow from Theorem 1 and Proposition 4 in [17]. If $\delta>0$ is such that $2+2 \delta \leq \alpha$, by simple calculations we obtain

$$
\left(\mathbb{E}_{x}\left|x_{n}\right|^{2+2 \delta}\right)^{\frac{1}{2+2 \delta}} \leq \varphi(2+2 \delta)^{\frac{n}{2+2 \delta}}|x|+\frac{\left\|b_{1}\right\|_{2+2 \delta}}{1-\varphi(2+2 \delta)^{\frac{1}{2+2 \delta}}} .
$$

Taking the sup in $n \geq 1$, we get

$$
\begin{aligned}
\mu_{\delta}(x) & =\sup _{n \geq 1}\left(\mathbb{E}_{x}\left|f\left(x_{n}\right)\right|^{2+2 \delta}\right)^{\frac{1}{2+2 \delta}} \\
& \leq \varphi(2+2 \delta)^{\frac{1}{2+2 \delta}}|x|+\frac{\left\|b_{1}\right\|_{2+2 \delta}}{1-\varphi(2+2 \delta)^{\frac{1}{2+2 \delta}}}
\end{aligned}
$$

which proves that hypothesis M4 is satisfied. Finally, hypothesis M5 is verified since

$$
\int \mu_{\delta}(x)^{2} \nu(d x) \leq 2\left(\varphi(2+2 \delta)^{\frac{1}{1+\delta}} \int x^{2} \nu(d x)+\left(\frac{\left\|b_{1}\right\|_{2+2 \delta}}{1-\varphi(2+2 \delta)^{\frac{1}{2+2 \delta}}}\right)^{2}\right)<\infty .
$$


The mean is given by $\nu(f)=\mathbb{E} Z=\sum_{i=1}^{\infty}\left(\mathbb{E} a_{1}\right)^{i-1} \mathbb{E} b_{1}=\frac{\mathbb{E} b_{1}}{1-\mathbb{E} a_{1}}$. Without loss of generality we can assume that $\nu(f)=0$, i.e. that $\mathbb{E} b_{1}=0$; then the variance is

$$
\sigma_{\nu}^{2}=\operatorname{Var}_{\mathbb{P}}(Z)=\lim _{n \rightarrow \infty} \mathbb{E}\left(\sum_{i=1}^{n} a_{1} \ldots a_{i-1} b_{i}\right)^{2}=\lim _{n \rightarrow \infty} \sum_{i=1}^{n}\left(\mathbb{E} a_{1}^{2}\right)^{i-1} \mathbb{E} b_{1}^{2}=\frac{\mathbb{E} b_{1}^{2}}{1-\mathbb{E} a_{1}^{2}}
$$

Then the conclusions of Theorem 3 hold true with $\mu=\nu(f)=0$ and $\sigma=\sigma_{\nu}^{2}=\frac{\mathbb{E} b_{1}^{2}}{1-\mathbb{E} a_{1}^{2}}$.

A multivariate version of the stochastic recursion has been considered in Guivarc' $h$ and Le Page [18] and can be treated in the same manner.

\section{Partition of the set $\mathbb{N}$ and notations}

In the sequel $\varepsilon, \beta \in(0,1)$ will be such that $\varepsilon+\beta<1$ (all over the paper $\varepsilon$ is supposed to be very small, while $\beta$ will be optimized). Denote for simplicity $[a, b)=\{l \in \mathbb{N}: a \leq l<b\}$. Let $k_{0} \geq 1$ be a natural number. We start by splitting the set $\mathbb{N}$ into subsets $\left[2^{k}, 2^{k+1}\right)$, $k=k_{0}, k_{0}+1, \ldots$ called blocks. Consider the $k$-th block $\left[2^{k}, 2^{k+1}\right)$. We leave a large gap $J_{k, 1}$ of length $2^{[\varepsilon k]+[\beta k]}$ at the left end of the $k$-th block. Then, following a triadic Cantor-like scheme, we split the remaining part $\left[2^{k}+2^{[\varepsilon k]+[\beta k]}, 2^{k+1}\right)$ into subsets $I_{k, j}$ and $J_{k, j}$ called islands and gaps as explained below. At the resolution level 0 a gap of size $2^{[\varepsilon k]+[\beta k]} / 2$ is put in the middle of the interval $\left[2^{k}+2^{[\varepsilon k]+[\beta k]}, 2^{k+1}\right)$. This yields two intervals of equal length. At the resolution level 1 two additional gaps of length $2^{[\varepsilon k]+[\beta k]} / 2^{2}$ are put in the middle of the each obtained interval which yields four intervals of equal length. Continuing in the same way, at the resolution level $[\beta k]$ we obtain $2^{[\beta k]}$ intervals $I_{k, j}, j=1, \ldots, 2^{[\beta k]}$ called islands and the same number of gaps $J_{k, j}, j=1, \ldots, 2^{[\beta k]}$ which we index from left to right (recall that $J_{k, 1}=J_{k, 2^{0}}$ denotes the large gap at the left end of the $k$-th block). It is obvious that $\left[2^{k}, 2^{k+1}\right)$ is the union of the constructed island and gaps, so that

$$
\left[2^{k}, 2^{k+1}\right)=J_{k, 1} \cup I_{k, 1} \cup \ldots \cup J_{k, 2^{[\beta k]}} \cup I_{k, 2^{[\beta k]}} .
$$

In the block $k$ there are one gap of the length $2^{[[\varepsilon k]]+[\beta k]}$ and $2^{l}$ gaps of length $2^{[[\varepsilon k]]+[\beta k]-l-1}$, where $l=0, \ldots,[\beta k]-1$. The length of the finest gap (for example $J_{k, 2^{[\beta k]}}$ ) is $2^{[\varepsilon k]}$. The total length of the gaps in the block $k$ is

$$
L_{k}^{g a p}=2^{[[\varepsilon k]]+[\beta k]}+\sum_{l=0}^{[\beta k]-1} 2^{l} 2^{[[\varepsilon k]]+[\beta k]-l-1}=(2+[\beta k]) 2^{[[\varepsilon k]]+[\beta k]-1} .
$$

Recall that, according to the construction, the islands of the $k$-th block have the same length

$$
\begin{aligned}
\left|I_{k, j}\right| & =\left(2^{k+1}-2^{k}-(2+[\beta k]) 2^{[[\varepsilon k]]+[\beta k]-1}\right) / 2^{[\beta k]} \\
& =2^{k-[\beta k]}-\left(1+[\beta k] 2^{[[\varepsilon k]]-1}\right) .
\end{aligned}
$$

An obvious upper bound is $\left|I_{k, j}\right| \leq 2^{k-[\beta k]}$. Since $\varepsilon<1-\beta$ we have $\left|I_{k, j}\right| \geq 2^{k-[\beta k]}-$ $2^{[[\varepsilon k]]-c_{\beta, \varepsilon}^{\prime} \ln k} \geq c_{\varepsilon, \beta} 2^{k(1-\beta)}$, with some $c_{\varepsilon, \beta} \in\left(0, \frac{1}{2}\right)$. Since the length of the $k$-th block is $2^{k}$ the total length of the islands of the $\mathrm{k}$-th block is equal to

$$
L_{k}^{i s l}=2^{k}-2^{[[\varepsilon k]]+[\beta k]-1}(2+[\beta k]) .
$$


Note that, for some constant $c_{\beta}>0$,

$$
c_{\beta} 2^{k} \leq L_{k}^{i s l} \leq 2^{k}
$$

Denote by $\mathcal{K}$ the set of double indices $(k, j)$, with $k=1,2, \ldots$ being the index of the block and $j=1, \ldots, 2^{[\beta k]}$ being the index of the island in the block $k$. The set $\mathcal{K}$ will be endowed with lexicographical order $\preceq$. Then the sets $I_{k, j}$ and $J_{k, j},(k, j) \in \mathcal{K}$, will be also endowed with the lexicographical order. Let $N \in \mathbb{N}$. From (4.1) it follows that there exists a unique $(n, m) \in \mathcal{K}$ such that $2^{n} \leq N<2^{n+1}$ and $N \in J_{n, m} \cup I_{n, m}$, where the dependence of $n$ and $m$ on $N$ is suppressed from the notation. Denote $\mathcal{K}_{N}=\{(k, j):(k, j) \preceq(n, m)\}$.

For ease of reading we recall the notations and properties that will be used throughout the paper:

P1: $\varepsilon$ and $\beta$ are positive numbers such that $\varepsilon+\beta<1$. Latter on, the constant $\varepsilon$ will be chosen to be small enough.

P2: $\mathcal{K}=\left\{(k, j): k=1,2, \ldots, j=1, \ldots, 2^{[\beta k]}\right\}$.

P3: For any $N \in \mathbb{N}$ the unique couple $(n, m) \in \mathcal{K}$ is such that $N \in J_{n, m} \cup I_{n, m}$.

P4: $\mathcal{K}_{N}=\{(k, j):(k, j) \preceq(n, m)\}$.

P5: $I_{k, j}, j=1, \ldots, 2^{[\beta k]}$ are the islands and $J_{k, j}, j=1, \ldots, 2^{[\beta k]}$ are the gaps in the $k$-th block.

P6: The number of islands and the number of gaps in the $k$-th block are both equal to $m_{k}=2^{[\beta k]}$. Set $m_{k, n}=m_{k}+\ldots+m_{n}$.

P7: The islands in $k$-th block have the same length $\left|I_{k, j}\right|=2^{k-[\beta k]}-\left(1+[\beta k] 2^{[[\varepsilon k]]-1}\right) \leq$ $2^{k-[\beta k]}$. This implies $\left|I_{k, j}\right| \geq c_{\varepsilon, \beta} 2^{k(1-\beta)}$ for some constant $c_{\varepsilon, \beta} \in\left(0, \frac{1}{2}\right)$.

P8: The length of the finest gap in the $k$-th block is $\left|J_{k, j}\right|=2^{[[\varepsilon k]]}$. This implies $\left|J_{k, j}\right| \geq 2^{[[\varepsilon k]]}$.

P9: The length $\left|J_{k, 1}\right|$ of the gap at the left end of the $k$-th block is $2^{[\varepsilon k]+[\beta k]}$.

P10: For each pair $(k, j) \in \mathcal{K}$, we denote $X_{(k, j)}=\sum_{i \in I_{k, j}} X_{i}$ and $W_{(k, j)}=\sum_{i \in I_{k, j}} W_{i}$.

P11: $\mathcal{L}_{X_{1}, \ldots, X_{d}}$ denotes the probability law of the random vector $\left(X_{1}, \ldots, X_{d}\right)$.

\section{Auxiliary Results}

Without loss of generality we assume that on the initial probability space there is a sequence of independent r.v.'s $\left(Y_{(k, j)}\right)_{(k, j) \in \mathcal{K}}$ such that $Y_{(k, j)} \stackrel{d}{=} X_{(k, j)},(k, j) \in \mathcal{K}$. Let $k_{0} \in \mathbb{N}_{+}$ and $n>k_{0}$. Suppose that on the same probability space there is an i.i.d. sequence of $\mathbb{R}^{1}$-valued r.v.'s $\left(V_{(k, j)}\right)_{(k, j) \in \mathcal{K}}$ with means 0 whose characteristic function has as support the interval $\left[-\varepsilon_{0}, \varepsilon_{0}\right]$ and such that $\mathbb{E}\left|V_{(k, j)}\right|^{r_{0}}<\infty$ for any $r_{0}>0$. We suppose that the sequence $\left(V_{(k, j)}\right)_{(k, j) \in \mathcal{K}}$ is independent of $\left(X_{(k, j)}\right)_{(k, j) \in \mathcal{K}}$ and $\left(Y_{(k, j)}\right)_{(k, j) \in \mathcal{K}}$. Denote $X_{(k)}=$ $\left(X_{(k, 1)}, \ldots, X_{\left(k, m_{k}\right)}\right), Y_{(k)}=\left(Y_{(k, 1)}, \ldots, Y_{\left(k, m_{k}\right)}\right)$ and $V_{(k)}=\left(V_{(k, 1)}, \ldots, V_{\left(k, m_{k}\right)}\right)$. In the sequel $\pi$ denotes the Prokhorov distance (for details see Section 7.1 of the Appendix).

The main result of this section is the following proposition, which is of independent interest. 
Proposition 3. Assume conditions $\boldsymbol{C 1}$ and $\boldsymbol{C 2}$. There thus exists a constant $c_{\varepsilon, \beta, \lambda_{1}, \lambda_{2}}$, depending only on its indices such that, for any $k_{0}=1,2, \ldots$ and $n>k_{0}$,

$$
\pi\left(\mathcal{L}_{X_{\left(k_{0}\right)}+V_{\left(k_{0}\right)}, \ldots, X_{(n)}+V_{(n)}}, \mathcal{L}_{Y_{\left(k_{0}\right)}+V_{\left(k_{0}\right)}, \ldots, Y_{(n)}+V_{(n)}}\right) \leq c_{\varepsilon, \beta, \lambda_{1}, \lambda_{2}}\left(1+\lambda_{0}+\mu_{\delta}\right) \exp \left(-\frac{\lambda_{1}}{4} 2^{\frac{\varepsilon}{2} k_{0}}\right) .
$$

The proof of Proposition 3 is based on Lemmas 1 and 2 below. Without loss of generality we assume that there exists a sequence of independent random vectors $R_{(k)}, k=1, \ldots, n$ such that $R_{(k)} \stackrel{d}{=} X_{(k)}+V_{(k)}$ and such that the sequence $\left(R_{(k)}\right)_{k=1, \ldots, n}$ is independent of the sequences $\left(X_{(k)}+V_{(k)}\right)_{k=1, \ldots, n},\left(Y_{(k, j)}\right)_{(k, j) \in \mathcal{K}}$ and $\left(V_{(k, j)}\right)_{(k, j) \in \mathcal{K}}$. The following proposition gives a bound for the Prokhorov distance between the vectors $\left(X_{\left(k_{0}\right)}+V_{\left(k_{0}\right)}, \ldots, X_{(n)}+V_{(n)}\right)$

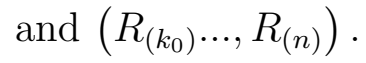

Lemma 1. Assume conditions $\boldsymbol{C 1}$ and $\boldsymbol{C 2}$. Then, there exists a constant $c_{\varepsilon, \beta, \lambda_{1}, \lambda_{2}}$, depending only on its indices such that, for any $k_{0}=1,2, \ldots$ and $n>k_{0}$,

$$
\pi\left(\mathcal{L}_{X_{\left(k_{0}\right)}+V_{\left(k_{0}\right)}, \ldots, X_{(n)}+V_{(n)}}, \mathcal{L}_{\left.R_{\left(k_{0}\right)}\right), R_{(n)}}\right) \leq c_{\varepsilon, \beta, \lambda_{1}, \lambda_{2}}\left(1+\lambda_{0}+\mu_{\delta}\right) \exp \left(-\frac{\lambda_{1}}{4} 2^{\frac{\varepsilon}{2} k_{0}}\right) .
$$

Proof. For $k=k_{0}, \ldots, n$, set

$$
Z_{(k)}=\left(X_{\left(k_{0}\right)}+V_{\left(k_{0}\right)}, \ldots, X_{(k)}+V_{(k)}\right) \quad \text { and } \quad \widetilde{Z}_{(k)}=\left(X_{\left(k_{0}\right)}+V_{\left(k_{0}\right)}, \ldots, X_{(k-1)}+V_{(k-1)}, R_{(k)}\right) .
$$

By Lemma 5, one gets

$$
\pi\left(\mathcal{L}_{X_{\left(k_{0}\right)}+V_{\left(k_{0}\right)}, \ldots, X_{(n)}+V_{(n)}}, \mathcal{L}_{\left.R_{\left(k_{0}\right)}\right), R_{(n)}}\right) \leq \sum_{k=k_{0}}^{n} \pi\left(\mathcal{L}_{Z_{(k)}}, \mathcal{L}_{\widetilde{Z}_{(k)}}\right) .
$$

Let $\phi_{(k)}$ (respectively $\left.\widetilde{\phi}_{(k)}\right)$ be the characteristic function of the vector $Z_{(k)}$ (respectively $\widetilde{Z}_{(k)}$ ) and $m_{k_{0}, k}=m_{k_{0}}+\ldots+m_{k}$. Then, by Lemma 7 , for any $T>0$,

$$
\begin{aligned}
\pi\left(\mathcal{L}_{Z_{(k)}}, \mathcal{L}_{\widetilde{Z}_{(k)}}\right) \leq & (T / \pi)^{m_{k_{0}, k} / 2}\left(\int_{t \in \mathbb{R}^{m_{k_{0}, k}}}\left|\phi_{(k)}(t)-\widetilde{\phi}_{(k)}(t)\right|^{2} d t\right)^{1 / 2} \\
& +\mathbf{P}\left(\max _{k_{0} \leq l \leq k} \max _{1 \leq j \leq m_{l}}\left|X_{(l, j)}\right|>T\right) .
\end{aligned}
$$

Denote by $\varphi_{(k)}$ (respectively $\left.\psi_{(k)}\right)$ the characteristic function of the vector $X_{(k)}$ (respectively $\left.\left(X_{\left(k_{0}\right)}, \ldots, X_{(k)}\right)\right)$. Since $V_{\left(k_{0}\right)}, \ldots, V_{(k)}$ are independent of $X_{\left(k_{0}\right)}, \ldots, X_{(k)}$ and $Y_{\left(k_{0}\right)}, \ldots, Y_{(k)}$, we have

$$
\begin{aligned}
& \int_{t \in \mathbb{R}^{m_{k_{0}, k}}}\left|\phi_{(k)}(t)-\varphi_{(k)}(t)\right|^{2} d t \\
= & \int_{t_{1} \in \mathbb{R}^{m_{k_{0}}}} \ldots \int_{t_{k} \in \mathbb{R}^{m_{k}}}\left|\phi_{(k)}\left(t_{k_{0}}, \ldots, t_{k}\right)-\varphi_{(k)}\left(t_{k_{0}}, \ldots, t_{k}\right)\right|^{2} d t_{k_{0}} \ldots d t_{k} \\
\leq & I_{1} \\
\equiv & \int_{t_{1} \in \mathbb{R}^{m_{k_{0}}}} \ldots \int_{t_{k} \in \mathbb{R}^{m_{k}}}\left|\psi_{(k)}\left(t_{k_{0}}, \ldots, t_{k}\right)-\psi_{(k-1)}\left(t_{k_{0}}, \ldots, t_{k-1}\right) \varphi_{(k)}\left(t_{k}\right)\right|^{2} d t_{k_{0}} \ldots d t_{k} .
\end{aligned}
$$


To bound the right-hand side of (5.3), note that $m_{k_{0}, k}=\left(2^{\left[\beta k_{0}\right]}+\ldots+2^{[\beta k]}\right) \leq 2^{[\beta k]+1}$ and, according to the construction, the length of the gap between the vectors $X_{(k-1)}$ and $X_{(k)}$ is $k_{\text {gap }}=2^{[\varepsilon k]+[\beta k]}$. Note also that $\left|I_{k, j}\right| \leq 2^{k-[\beta k]}$ and $\left|\varepsilon_{0}\right| \leq 1$. Let us remind that the characteristic functions of the r.v. $V_{(k, j)}$ have support the interval $\left[-\epsilon_{0}, \epsilon_{0}\right]$ and that the sequence $\left(V_{(k, j)}\right)_{(k, j) \in \mathcal{K}}$ is independent of $\left(X_{(k, j)}\right)_{(k, j) \in \mathcal{K}}$; it readily implies that the intregrals above are in fact over the set $\left[-\epsilon_{0}, \epsilon_{0}\right]^{m_{k_{0}, k}}$. Using condition C1, with $M_{1}=m_{k_{0}, k-1}$ and $M_{2}=m_{k}$, one may thus write

$$
\begin{aligned}
I_{1} & \leq \lambda_{0}\left(1+\max _{l \leq k, j \leq m_{k}}\left|I_{l, j}\right|\right)^{\lambda_{2}\left(M_{1}+M_{2}\right)} \exp \left(-\lambda_{1} k_{g a p}\right) \varepsilon_{0}^{m_{k_{0}, k}} \\
& \leq \lambda_{0}\left(1+2^{k-[\beta k]}\right)^{\lambda_{2} 2^{[\beta k]+1}} \exp \left(-\lambda_{1} k_{g a p}\right) \\
& \leq \lambda_{0} \exp \left(-\lambda_{1} 2^{[\varepsilon k]+[\beta k]}+\lambda_{2} 2^{[\beta k]+1} \ln \left(1+2^{k-[\beta k]}\right)\right) \\
& \leq c_{\varepsilon, \beta, \lambda_{1}, \lambda_{2}} \lambda_{0} \exp \left(-\frac{\lambda_{1}}{2} 2^{[\varepsilon k]+[\beta k]}\right) .
\end{aligned}
$$

Putting together (5.2), (5.3) and (5.4), we get

$$
\begin{aligned}
\pi\left(\mathcal{L}_{Z_{(k)}}, \mathcal{L}_{\widetilde{Z}_{(k)}}\right) \leq & c_{\varepsilon, \beta, \lambda_{1}, \lambda_{2}} \lambda_{0}(T / \pi)^{m_{k_{0}, k} / 2} \exp \left(-\frac{\lambda_{1}}{2} 2^{[\varepsilon k]+[\beta k]}\right) \\
& +\sum_{k_{0} \leq l \leq k} \sum_{1 \leq j \leq m_{l}} \mathbb{P}\left(\left|X_{(l, j)}\right|>T\right) .
\end{aligned}
$$

Since $\left|I_{(l, j)}\right| \leq 2^{l}$, by Markov's inequality and condition C2,

$$
\mathbb{P}\left(\left|X_{(l, j)}\right|>T\right) \leq T^{-1} \mathbb{E}\left|X_{(l, j)}\right| \leq T^{-1} 2^{l} \max _{i} \mathbb{E}\left|X_{i}\right| \leq \mu_{\delta} T^{-1} 2^{l} .
$$

Choosing $T=\exp \left(2^{[\varepsilon k] / 2}\right)$, one gets

$$
\begin{aligned}
\sum_{k_{0} \leq l \leq k} \sum_{1 \leq j \leq m_{l}} \mathbb{P}\left(\left|X_{(l, j)}\right|>T\right) & \leq \mu_{\delta} T^{-1} \sum_{k_{0} \leq l \leq k} m_{l} 2^{l} \\
& \leq \mu_{\delta} \exp \left(-2^{[\varepsilon k] / 2}\right) \sum_{k_{0} \leq l \leq k} 2^{[\beta l]} 2^{l} \\
& \leq c_{\beta} \mu_{\delta} \exp \left(-2^{[\varepsilon k] / 2} / 2\right) .
\end{aligned}
$$

Since $m_{k_{0}, k} \leq 2^{\beta k}$, one gets

$$
(T / \pi)^{m_{k_{0}, k} / 2} \leq \exp \left(\frac{1}{2} 2^{[\varepsilon k] / 2+\beta k}\right) .
$$

From (5.5), (5.6) and (5.7), we deduce

$$
\begin{aligned}
\pi\left(\mathcal{L}_{Z_{(k)}}, \mathcal{L}_{\widetilde{Z}_{(k)}}\right) \leq & c_{\varepsilon, \beta, \lambda_{1}, \lambda_{2}} \lambda_{0} \exp \left(\frac{1}{2} 2^{[\varepsilon k] / 2+[\beta k]}\right) \exp \left(-\frac{\lambda_{1}}{2} 2^{[\varepsilon k]+[\beta k]}\right) \\
& +c_{\beta} \mu_{\delta} \exp \left(-2^{[\varepsilon k] / 2} / 2\right) \\
\leq & \left(1+\lambda_{0}+\mu_{\delta}\right) c_{\varepsilon, \beta, \lambda_{1}, \lambda_{2}} \exp \left(-\frac{\lambda_{1}}{4} 2^{\varepsilon k / 2}\right) .
\end{aligned}
$$


Using (5.1)

$$
\begin{aligned}
\pi\left(\mathcal{L}_{\left(X_{\left(k_{0}\right)}+V_{\left(k_{0}\right)}, \ldots, X_{(n)}+V_{(n)}\right)}, \mathcal{L}_{\left(R_{\left(k_{0}\right)} \ldots, R_{(n)}\right)}\right) & \leq\left(1+\lambda_{0}+\mu_{\delta}\right) c_{\varepsilon, \beta, \lambda_{1}, \lambda_{2}} \sum_{k=k_{0}}^{n} \exp \left(-\frac{\lambda_{1}}{4} 2^{[\varepsilon k] / 2}\right) \\
& \leq\left(1+\lambda_{0}+\mu_{\delta}\right) c_{\varepsilon, \beta, \lambda_{1}, \lambda_{2}}^{\prime} \exp \left(-\frac{\lambda_{1}}{4} 2^{[\varepsilon k]_{0} / 2}\right) .
\end{aligned}
$$

This concludes the proof of Lemma 1.

The following assertion gives a bound for the Prokhorov distance between the vectors $\left(R_{\left(k_{0}\right)}, \ldots, R_{(n)}\right)$ and $\left(Y_{\left(k_{0}\right)}+V_{\left(k_{0}\right)}, \ldots, Y_{(n)}+V_{(n)}\right)$.

Lemma 2. Assume conditions $\boldsymbol{C 1}$ and $\boldsymbol{C 2}$. There exists a constant $c_{\varepsilon, \beta, \lambda_{1}, \lambda_{2}}$, depending only on its indices, such that, for any $k_{0}=1,2, \ldots$ and $n>k_{0}$,

$$
\pi\left(\mathcal{L}_{R_{\left(k_{0}\right)}, \ldots, R_{(n)}}, \mathcal{L}_{Y_{\left(k_{0}\right)}+V_{\left(k_{0}\right)}, \ldots, Y_{(n)}+V_{(n)}}\right) \leq c_{\varepsilon, \beta, \lambda_{1}, \lambda_{2}}\left(1+\lambda_{0}+\mu_{\delta}\right) \exp \left(-\frac{\lambda_{1}}{8} 2^{[\varepsilon k]_{0} / 2}\right) .
$$

Proof. By Lemma 6, since $R_{\left(k_{0}\right)}, \ldots, R_{(n)}$ and $Y_{\left(k_{0}\right)}+V_{\left(k_{0}\right)}, \ldots, Y_{(n)}+V_{(n)}$ are independent r.v.'s, one may write

$$
\pi\left(\mathcal{L}_{R_{\left(k_{0}\right)}, \ldots, R_{(n)}}, \mathcal{L}_{Y_{\left(k_{0}\right)}+V_{\left(k_{0}\right)}, \ldots, Y_{(n)}+V_{(n)}}\right)=\sum_{k=k_{0}}^{n} \pi\left(\mathcal{L}_{R_{(k)}}, \mathcal{L}_{Y_{(k)}+V_{(k)}}\right)
$$

and its suffices to prove that, for any $k=1,2, \ldots$, one gets

$$
\pi\left(\mathcal{L}_{R_{(k)}}, \mathcal{L}_{Y_{(k)}+V_{(k)}}\right) \leq\left(1+\lambda_{0}+\mu_{\delta}\right) c_{\varepsilon, \beta, \lambda_{1}, \lambda_{2}}^{\prime} \exp \left(-\frac{\lambda_{1}}{8} 2^{[\varepsilon k] / 2}\right)
$$

For this, recall that, according to the construction in Section 4, at the resolution level 0, a gap of length $2^{[[\varepsilon k]]+[\beta k]} / 2$ in the middle of the block $R_{(k)}^{0,0}=R_{(k)}$ splits it into two vectors $\widetilde{R}_{(k)}^{0,1}$ and $\widetilde{R}_{(k)}^{0,2}$; let $R_{(k)}^{0,1}$ and $R_{(k)}^{0,2}$ be independent versions of $\widetilde{R}_{(k)}^{0,1}$ and $\widetilde{R}_{(k)}^{0,2}$ respectively. Next, at the resolution level 1 , for any $j \in\{1,2\}$, a gap of length $2^{[(\varepsilon+\beta) k]} / 4$ in the middle of the block $R_{(k)}^{0, j}$ splits it into two vectors $\widetilde{R}_{(k)}^{1,2 j-1}$ and $\widetilde{R}_{(k)}^{1,2 j}$; let $R_{(k)}^{1,2 j-1}$ and $R_{(k)}^{1,2 j}$ be independent versions of $\widetilde{R}_{(k)}^{1,2 j-1}$ and $\widetilde{R}_{(k)}^{1,2 j}$ respectively. Assuming that at the resolution level $l \in\{1, \ldots,[\beta k]\}$ the independent r.v.'s $R_{(k)}^{l, j}, j \in\left\{1, \ldots, 2^{l}\right\}$, are already constructed, we shall perform the construction at the resolution level $l+1$. Note that, at the resolution level $l$ for any $j \in$ $\left\{1, \ldots, 2^{l}\right\}$, a gap of length $2^{[(\varepsilon+\beta) k]} / 2^{l+1}$ in the middle of the block $R_{(k)}^{l, j}$ splits it into two vectors $\widetilde{R}_{(k)}^{l+1,2 j-1}$ and $\widetilde{R}_{(k)}^{l+1,2 j}$; it is enough to set $R_{(k)}^{l+1,2 j-1}$ and $R_{(k)}^{l+1,2 j}$ to be independent versions of $\widetilde{R}_{(k)}^{l+1,2 j-1}$ and $\widetilde{R}_{(k)}^{l+1,2 j}$ respectively. It is easy to see that at the final level $l_{k}=[\beta k]$ we have $R_{(k)}^{l_{k}, j} \stackrel{d}{=} Y_{(k, j)}+V_{(k, j)}$, for $j=1, \ldots, m_{k}=2^{[\beta k]}$.

Let $l \in\{0, \ldots,[\beta k]\}$. For $j \in\left\{1, \ldots, 2^{l}\right\}$, denote by $\psi_{k}^{l, 2 j-1}$ and $\psi_{k}^{l, 2 j}$ the characteristic functions of $R_{(k)}^{l, 2 j-1}$ and $R_{(k)}^{l, 2 j}$. Using Lemma 7 and the independence of the vectors $\widetilde{R}_{(k)}^{l, 2 j-1}$ 
and $\widetilde{R}_{(k)}^{l, 2 j}$, we get

$$
\begin{aligned}
& \pi\left(\mathcal{L}_{R_{(k)}^{l, j}} \mathcal{L}_{R_{(k)}^{l+1,2 j-1}, R_{(k)}^{l+1,2 j}}\right) \\
& \leq\left((T / \pi)^{2^{-l} m_{k}} \int_{(t, s) \in \mathbb{R}^{2-l} m_{k}}\left|\psi_{k}^{l, j}(t, s)-\psi_{k}^{l+1,2 j-1}(t) \psi_{k}^{l+1,2 j}(s)\right|^{2} d t d s\right)^{1 / 2} \\
& \quad+\sum_{1 \leq j \leq 2^{-l} m_{k}} \mathbb{P}\left(\left|X_{(k, j)}+V_{(k, j)}\right|>T\right) .
\end{aligned}
$$

By Condition C1 with $N=M=\frac{m_{k}}{2} 2^{-l}$ and $k_{\text {gap }}=2^{[\varepsilon k]+[\beta k]-l-1}$, we obtain

$$
\begin{aligned}
& \int_{(t, s) \in \mathbb{R}^{m_{k}{ }^{-l}}}\left|\psi_{k}^{l, j}(t, s)-\psi_{k}^{l+1,2 j-1}(t) \psi_{k}^{l+1,2 j}(s)\right|^{2} d t d s \\
= & \int_{(t, s) \in \mathbb{R}^{m_{k^{2}}-l},\|t\|_{\infty} \leq \varepsilon_{0},\|s\|_{\infty} \leq \varepsilon_{0}}\left|\psi_{k}^{l, j}(t, s)-\psi_{k}^{l+1,2 j-1}(t) \psi_{k}^{l+1,2 j}(s)\right|^{2} d t d s \\
\leq & \lambda_{0} \exp \left(\lambda_{2} m_{k} 2^{-l} \ln \left(1+2^{k-[\beta k]}\right)-\lambda_{1} 2^{[\varepsilon k]+[\beta k]-1-l}\right)\left(2 \varepsilon_{0}\right)^{m_{k} 2^{-l}} \\
\leq & \lambda_{0} c_{\varepsilon, \beta, \lambda_{1}, \lambda_{2}}^{\prime \prime} \exp \left(-\frac{\lambda_{1}}{4} 2^{[\varepsilon k]+[\beta k]-l}\right) .
\end{aligned}
$$

We will thus take $T=\exp \left(\lambda_{1} 2^{[\varepsilon k]] / 2}\right)$ so that

$$
(T / \pi)^{2^{-l} m_{k}} \leq \exp \left(\lambda_{1} 2^{-l} m_{k} 2^{[[\varepsilon k]] / 2}\right) \leq \exp \left(\lambda_{1} 2^{[[\varepsilon k]] / 2+[\beta k]-l}\right)
$$

In order to control the terms $\mathbb{P}\left(\left|X_{(k, j)}+V_{(k, j)}\right|>T\right)$, we use Markov's inequality, condition C2 and the fact that $\left|I_{k, j}\right| \leq 2^{k}$; it readily follows that

$$
\begin{aligned}
\mathbb{P}\left(\left|X_{(k, j)}+V_{(k, j)}\right|>T\right) & \leq T^{-1}\left(\mathbb{E}\left|X_{(k, j)}\right|+\mathbb{E}\left|V_{(k, j)}\right|\right) \\
& \leq T^{-1}\left(2^{k} \max _{i} \mathbb{E}\left|X_{i}\right|+c 2^{k}\right) \\
& \leq\left(1+\mu_{\delta}\right) c 2^{k} \exp \left(-\lambda_{1} 2^{-[[\varepsilon k]] / 2}\right)
\end{aligned}
$$

Therefore

$$
\begin{aligned}
\sum_{1 \leq j \leq 2^{-l} m_{k}} \mathbb{P}\left(\left|X_{(k, j)}+V_{(k, j)}\right|>T\right) & \leq 2^{-l} m_{k}\left(1+\mu_{\delta}\right) c 2^{k} \exp \left(-2^{[[\varepsilon k]] / 2}\right) \\
& \leq\left(1+\mu_{\delta}\right) \exp \left(-\lambda_{1} 2^{[[\varepsilon k]] / 2}\right) 2^{-l} 2^{2[\beta k]+k} \\
& \leq\left(1+\mu_{\delta}\right) c_{\varepsilon, \beta, \lambda_{1}, \lambda_{2}} \exp \left(-\frac{\lambda_{1}}{2} 2^{[[\varepsilon k]] / 2}\right) .
\end{aligned}
$$


From (5.10), (5.11) and (5.12), we get

$$
\begin{aligned}
& \pi\left(\mathcal{L}_{R_{(k)}^{l, j}}, \mathcal{L}_{R_{(k)}^{l+1,2 j-1}, R_{(k)}^{l+1,2 j}}\right) \\
\leq & \left(1+\lambda_{0}+\mu_{\delta}\right) c_{\varepsilon, \beta, \lambda_{1}, \lambda_{2}} \\
& \times\left[\exp \left(\lambda_{1} 2^{-l} 2^{[[\varepsilon k]] / 2+[\beta k]}\right) \exp \left(-\frac{\lambda_{1}}{2} 2^{-l} 2^{[\varepsilon k]+[\beta k]}\right)+c \exp \left(-\frac{\lambda_{1}}{2} 2^{[[\varepsilon k]] / 2}\right)\right] \\
(5.13) \leq & \left(1+\lambda_{0}+\mu_{\delta}\right) c_{\varepsilon, \beta, \lambda_{1}, \lambda_{2}} \exp \left(-\frac{\lambda_{1}}{4} 2^{[[\varepsilon k]] / 2}\right) .
\end{aligned}
$$

Since $R_{(k)}^{l, j}, j=1, \ldots, 2^{l}$ are independent r.v.'s, by triangle inequality, one gets

$$
\begin{aligned}
& \pi\left(\mathcal{L}_{R_{(k)}}, \mathcal{L}_{Y_{(k)}+V_{(k)}}\right)=\pi\left(\mathcal{L}_{R_{(k)}^{0,0}}, \mathcal{L}_{Y_{(k)}+V_{(k)}}\right) \\
& \leq \pi\left(\mathcal{L}_{R_{(k)}^{0,0}}, \mathcal{L}_{R_{(k)}^{0,1}, R_{(k)}^{0,2}}\right)+\pi\left(\mathcal{L}_{R_{(k)}^{0,1}, R_{(k)}^{0,2}}, \mathcal{L}_{Y_{(k)}+V_{(k)}}\right) \\
& \leq \pi\left(\mathcal{L}_{R_{(k)}^{0,0}}, \mathcal{L}_{R_{(k)}^{0,1}, R_{(k)}^{0,2}}\right)+\pi\left(\mathcal{L}_{R_{(k)}^{0,1}, R_{(k)}^{0,2}}, \mathcal{L}_{R_{(k)}^{1,1}, \ldots, R_{(k)}^{1,4}}\right) \\
& +\pi\left(\mathcal{L}_{R_{(k)}^{1,1}, \ldots, R_{(k)}^{1,4}}, \mathcal{L}_{Y_{(k)}+V_{(k)}}\right) \\
& \leq \sum_{l=0}^{[\beta k]-1} \pi\left(\mathcal{L}_{R_{(k)}^{l, 1}, \ldots, R_{(k)}^{l, 2 l}}, \mathcal{L}_{R_{(k)}^{l+1,1}, \ldots, R_{(k)}^{l+1,2^{l+1}}}\right) .
\end{aligned}
$$

By Lemma 6 and (5.13) one gets

$$
\begin{aligned}
\pi\left(\mathcal{L}_{R_{(k)}^{l, 1}, \ldots, R_{(k)}^{l, 2 l}}, \mathcal{L}_{R_{(k)}^{l+1,1}, \ldots, R_{(k)}^{l+1,2^{l+1}}}\right) & \leq \sum_{j=1}^{2^{l}} \pi\left(\mathcal{L}_{R_{(k)}^{l, j}}, \mathcal{L}_{R_{(k)}^{l+1,2 j-1}, R_{(k)}^{l+1,2 j}}\right) \\
& \leq 2^{l}\left(1+\lambda_{0}+\mu_{\delta}\right) c_{\varepsilon, \beta, \lambda_{1}, \lambda_{2}} \exp \left(-\frac{\lambda_{1}}{4} 2^{[\varepsilon k] / 2}\right) .
\end{aligned}
$$

From (5.14) and (5.15), it follows

$$
\begin{aligned}
\pi\left(\mathcal{L}_{R_{(k)}}, \mathcal{L}_{Y_{(k)}+V_{(k)}}\right) & \leq \sum_{l=0}^{[\beta k]-1} 2^{l}\left(1+\lambda_{0}+\mu_{\delta}\right) c_{\varepsilon, \beta, \lambda_{1}, \lambda_{2}} \exp \left(-\frac{\lambda_{1}}{4} 2^{[\varepsilon k] / 2}\right) \\
& \leq 2^{[\beta k]}\left(1+\lambda_{0}+\mu_{\delta}\right) c_{\varepsilon, \beta, \lambda_{1}, \lambda_{2}} \exp \left(-\frac{\lambda_{1}}{4} 2^{[\varepsilon k] / 2}\right) \\
& \leq\left(1+\lambda_{0}+\mu_{\delta}\right) c_{\varepsilon, \beta, \lambda_{1}, \lambda_{2}}^{\prime} \exp \left(-\frac{\lambda_{1}}{8} 2^{[\varepsilon k] / 2}\right) .
\end{aligned}
$$

Finally, (5.9) holds; this finishes the proof of Lemma 2.

Proposition 3 follows from Lemmas 1 and 2 by triangle inequality. 


\section{Proof of TheOrem 1}

This section is devoted to the proof of Theorem 1; it is separated in several steps. We first construct the coupling with independent r.v.'s. (Section 6.1) and thus with independent normal r.v.'s. (Section 6.2). In Section 6.3, we explicit the construction of the sequences $\left(\widetilde{X}_{i}\right)_{1 \leq i \leq N}$ and $\left(W_{i}\right)_{1 \leq i \leq N}$ and in Sections $6.4,6.5,6.6$ and 6.7 we put together and optimize the bounds.

6.1. Coupling with independent r.v.'s. The following proposition shows that with high probability the partial sums $\sum_{(l, i) \preceq(k, j)} X_{(l, i)}$ can be coupled with the partial sums $\sum_{(l, i) \preceq(k, j)} Y_{(l, i)}$.

Proposition 4. Assume conditions $\boldsymbol{C 1}$ and $\boldsymbol{C 2}$. Let $\alpha<\delta, \beta>\frac{1}{2}$ and $0<\rho<\frac{1-\beta}{2}$. Then, for any $N \in \mathbb{N}$, on some extension of the initial probability space there is a version $\left(X_{(k, j)}^{\prime}\right)_{(k, j) \in \mathcal{K}_{N}}$ of the sequence $\left(X_{(k, j)}\right)_{(k, j) \in \mathcal{K}_{N}}$ and a version $\left(Y_{(k, j)}^{\prime}\right)_{(k, j) \in \mathcal{K}_{N}}$ of the sequence $\left(Y_{(k, j)}\right)_{(k, j) \in \mathcal{K}_{N}}$ such that

$$
\begin{aligned}
& \mathbb{P}\left(\left(2^{n}\right)^{-\frac{1}{2}} \sup _{(k, j) \in \mathcal{K}_{N}}\left|\sum_{(l, i) \preceq(k, j)}\left(X_{(l, i)}^{\prime}-Y_{(l, i)}^{\prime}\right)\right| \geq\left(2^{n}\right)^{-\rho}\right) \\
\leq & C_{1}\left(2^{n}\right)^{-1-\alpha+(\varepsilon+\rho)(2+2 \alpha)},
\end{aligned}
$$

where $\varepsilon \in\left(0, \frac{1}{2}\right)$ is arbitrary choosen and $C_{1}=c_{\varepsilon, \beta, \lambda_{1}, \lambda_{2}, \alpha, \rho}\left(1+\lambda_{0}+\mu_{\delta}\right)^{2+2 \delta}$ for some positive constant $c_{\varepsilon, \beta, \lambda_{1}, \lambda_{2}, \alpha, \rho}$ depending only on its indices.

Proof. For the sake of brevity, it is convenient to set $k_{0}=[\varepsilon n], X_{k_{0}, n}=\left(X_{\left(k_{0}\right)}, \ldots, X_{(n)}\right), Y_{k_{0}, n}=$ $\left(Y_{\left(k_{0}\right)}, \ldots, Y_{(n)}\right)$ and $V_{k_{0}, n}=\left(V_{\left(k_{0}\right)}, \ldots, V_{(n)}\right)$. Let $\widetilde{X}_{k_{0}, n}=X_{k_{0}, n}+V_{k_{0}, n}$ and $\widetilde{Y}_{k_{0}, n}=Y_{k_{0}, n}+V_{k_{0}, n}$ be the smoothed versions of $X_{k_{0}, n}$ and $Y_{k_{0}, n}$. By Proposition 3, with $k_{0}=[\varepsilon n]$, there exists a constant $c_{\varepsilon, \beta, \lambda_{1}, \lambda_{2}}$ such that

$$
\pi\left(\mathcal{L}_{\widetilde{X}_{k_{0}, n}}, \mathcal{L}_{\widetilde{Y}_{k_{0}, n}}\right) \leq \Delta=\left(1+\lambda_{0}+\mu_{\delta}\right) c_{\varepsilon, \beta, \lambda_{1}, \lambda_{2}} \exp \left(-\frac{\lambda_{1}}{4} 2^{\varepsilon^{2} n / 2}\right)
$$

Using Strassen-Dudley's theorem (see Lemma 3), we conclude that on some extension of the initial probability space there are random vectors $\widetilde{S}_{k_{0}, n}=\left(S_{\left(k_{0}\right)}, \ldots, S_{(n)}\right)$ and $\widetilde{T}_{k_{0}, n}=$ $\left(T_{\left(k_{0}\right)}, \ldots, T_{(n)}\right)$ such that $\widetilde{S}_{k_{0}, n} \stackrel{d}{=} \widetilde{X}_{k_{0}, n}, \widetilde{T}_{k_{0}, n} \stackrel{d}{=} \widetilde{Y}_{k_{0}, n}$ and

$$
\mathbb{P}\left(\left\|\widetilde{S}_{k_{0}, n}-\widetilde{T}_{k_{0}, n}\right\|_{\infty} \geq \Delta\right) \leq \Delta .
$$

We shall remove the smoothig from the vectors $\widetilde{S}_{k_{0}, n}$ and $\widetilde{T}_{k_{0}, n}$. Without loss of generality we consider that there is a random vector $U$ with uniform distribution on $[0,1]^{m_{k_{0}, n}}$ and independent of $\left(\widetilde{S}_{k_{0}, n}, \widetilde{T}_{k_{0}, n}\right)$. Consider the transition kernels $G_{1}(x \mid y)=\mathbb{P}\left(X_{k_{0}, n} \leq x \mid \widetilde{X}_{k_{0}, n}=y\right)$ and $G_{2}(x \mid y)=\mathbb{P}\left(Y_{k_{0}, n} \leq x \mid \widetilde{Y}_{k_{0}, n}=y\right)$. Define $X_{k_{0}, n}^{\prime}=G_{1}^{-1}\left(U \mid \widetilde{S}_{k_{0}, n}\right), V_{k_{0}, n}^{\prime}=\widetilde{S}_{k_{0}, n}-X_{k_{0}, n}^{\prime}$ and $Y_{k_{0}, n}^{\prime}=G_{1}^{-1}\left(U \mid T_{k_{0}, n}\right), V_{k_{0}, n}^{\prime \prime}=\widetilde{T}_{k_{0}, n}-Y_{k_{0}, n}^{\prime}$. The two sequences $X_{k_{0}, n}^{\prime}$ and $Y_{k_{0}, n}^{\prime}$ are such that $\widetilde{S}_{k_{0}, n}=X_{k_{0}, n}^{\prime}+V_{k_{0}, n}^{\prime}, \widetilde{T}_{k_{0}, n}=Y_{k_{0}, n}^{\prime}+V_{k_{0}, n}^{\prime \prime}$ and $X_{k_{0}, n}^{\prime} \stackrel{d}{=} X_{k_{0}, n}, Y_{k_{0}, n}^{\prime} \stackrel{d}{=} Y_{k_{0}, n}$, $V_{k_{0}, n}^{\prime} \stackrel{d}{=} V_{k_{0}, n}^{\prime \prime} \stackrel{d}{=} V_{k_{0}, n}$. The coordinates of the vectors $X_{k_{0}, n}^{\prime}$ and $Y_{k_{0}, n}^{\prime}$ are denoted by $X_{(k, j)}^{\prime}$ 
and $Y_{(k, j)}^{\prime},(k, j) \in \mathcal{K}$. Since $\widetilde{S}_{(k, j)}=X_{(k, j)}^{\prime}+V_{(k, j)}^{\prime}$ and $\widetilde{T}_{(k, j)}=Y_{(k, j)}^{\prime}+V_{(k, j)}^{\prime \prime}$, we have, for any $x \geq 1$,

$$
R=\mathbb{P}\left(\sup _{k_{0} \leq k,(k, j) \in \mathcal{K}_{N}}\left|\sum_{(l, i) \preceq(k, j)}\left(X_{(l, i)}^{\prime}-Y_{(l, i)}^{\prime}\right)\right| \geq 2 x\right) \leq R_{1}+R_{2},
$$

where

$$
\begin{aligned}
& R_{1}=\mathbb{P}\left(\sup _{k_{0} \leq k,(k, j) \in \mathcal{K}_{N}}\left|\sum_{(l, i) \preceq(k, j)} \widetilde{S}_{(l, i)}-\widetilde{T}_{(l, i)}\right| \geq x\right), \\
& R_{2}=\mathbb{P}\left(\sup _{k_{0} \leq k,(k, j) \in \mathcal{K}_{N}}\left|\sum_{(l, i) \preceq(k, j)}\left(V_{(l, i)}^{\prime}-V_{(l, i)}^{\prime \prime}\right)\right| \geq x\right) .
\end{aligned}
$$

First, we shall give a control for $R_{1}$. Note that card $\mathcal{K}_{N} \leq c 2^{\beta n}$. For any sequence of positive numbers $\left(\alpha_{(k, j)}\right)_{(k, j) \in \mathcal{K}}$ such that $\sum_{(k, j) \in \mathcal{K}} \alpha_{(k, j)} \leq 1$, it holds

$$
\begin{aligned}
& \left\{\sup _{k_{0} \leq k,(k, j) \in \mathcal{K}_{N}}\left|\sum_{k_{0} \leq l,(l, i) \preceq(k, j)}\left(\widetilde{S}_{(l, i)}-\widetilde{T}_{(l, i)}\right)\right| \geq x\right\} \\
\subseteq & \bigcup_{k_{0} \leq k,(k, j) \in \mathcal{K}_{N}}\left\{\left|\sum_{k_{0} \leq l,(l, i) \preceq(k, j)}\left(\widetilde{S}_{(l, i)}-\widetilde{T}_{(l, i)}\right)\right| \geq x\right\} \\
\subseteq & \bigcup_{k_{0} \leq k,(k, j) \in \mathcal{K}_{N}}\left\{\widetilde{k}_{0} \leq k,(l, i) \preceq(k, j)\right. \\
= & \bigcup_{k_{0} \leq k,(k, j) \in \mathcal{K}_{N}}\left\{\left|\widetilde{S}_{(k, j)}-\widetilde{T}_{(k, j)}\right| \geq x \alpha_{(k, j)}\right\},
\end{aligned}
$$

which implies that

$$
R_{1} \leq \sum_{k_{0} \leq k,(k, j) \in \mathcal{K}_{N}} \mathbb{P}\left(\left|\widetilde{S}_{(k, j)}-\widetilde{T}_{(k, j)}\right| \geq x \alpha_{(k, j)}\right) .
$$

Let $p=2+2 \alpha<2+2 \delta$. By Chebyshev's inequality

$$
R_{1} \leq x^{-p} \sum_{k_{0} \leq k,(k, j) \in \mathcal{K}_{N}} \alpha_{(k, j)}^{-p} \mathbb{E}\left|\widetilde{S}_{(k, j)}-\widetilde{T}_{(k, j)}\right|^{p} .
$$

By a truncation argument, with $\Delta$ from (6.1) and (6.2),

$$
\begin{aligned}
R_{1} \leq & x^{-p} \Delta^{p} \sum_{k_{0} \leq k,(k, j) \in \mathcal{K}_{N}} \alpha_{(k, j)}^{-p} \\
& +x^{-p} \sum_{k_{0} \leq k,(k, j) \in \mathcal{K}_{N}} \alpha_{(k, j)}^{-p} \mathbb{E}\left|\widetilde{S}_{(k, j)}-\widetilde{T}_{(k, j)}\right|^{p} 1\left(\left|\widetilde{S}_{(k, j)}-\widetilde{T}_{(k, j)}\right| \geq \Delta\right) .
\end{aligned}
$$


Let $\eta \in(0, \delta-\alpha), p^{\prime}=p+2 \eta$ and $\gamma=\frac{2 \eta}{p+2 \eta} \leq \eta$. Applying Hölder's inequality one may write $\left\|\left|\widetilde{S}_{(k, j)}-\widetilde{T}_{(k, j)}\right| 1\left(\left|\widetilde{S}_{(k, j)}-\widetilde{T}_{(k, j)}\right| \geq \Delta\right)\right\|_{L^{p}} \leq\left\|\widetilde{S}_{(k, j)}-\widetilde{T}_{(k, j)}\right\|_{L^{p^{\prime}}} \mathbb{P}\left(\left|\widetilde{S}_{(k, j)}-\widetilde{T}_{(k, j)}\right|>\Delta\right)^{\frac{\gamma}{p}}$

By Condition C2, for some constant $c>0$, we get

$$
\left\|\widetilde{S}_{(k, j)}-\widetilde{T}_{(k, j)}\right\|_{L^{p^{\prime}}} \leq 2\left\|X_{(k, j)}\right\|_{L^{p^{\prime}}}+2\left\|V_{(k, j)}^{\prime}\right\|_{L^{p^{\prime}}} \leq c\left(1+\mu_{\delta}\right)\left|I_{k, j}\right|
$$

consequently, using (6.2)

$$
\begin{aligned}
R_{1} \leq & x^{-p} \Delta^{p} \sum_{k_{0} \leq k,(k, j) \in \mathcal{K}_{N}} \alpha_{(k, j)}^{-p} \\
& +c\left(1+\mu_{\delta}\right)^{p} x^{-p} \sum_{k_{0} \leq k,(k, j) \in \mathcal{K}_{N}} \alpha_{(k, j)}^{-p}\left|I_{k, j}\right|^{p}\left(\mathbf{P}\left(\left|\widetilde{S}_{(k, j)}-\widetilde{T}_{(k, j)}\right| \geq \Delta\right)\right)^{\gamma} \\
\leq & x^{-p} \Delta^{p} \sum_{k_{0} \leq k,(k, j) \in \mathcal{K}_{N}} \alpha_{(k, j)}^{-p} \\
& +c\left(1+\mu_{\delta}\right)^{p} x^{-p} \Delta^{\gamma} \sum_{k_{0} \leq k,(k, j) \in \mathcal{K}_{N}} \alpha_{(k, j)}^{-p} 2^{(k-[\beta k]) p} \\
\leq & c_{\varepsilon, \beta, \lambda_{1}, \lambda_{2}, \eta}\left(1+\lambda_{0}+\mu_{\delta}\right)^{p+\gamma} \exp \left(-\frac{\lambda_{1}}{4} \gamma 2^{\varepsilon^{2} n / 2}\right) x^{-p} \sum_{k_{0} \leq k \leq n} \sum_{j \leq 2[\beta k]} \alpha_{(k, j)}^{-p} 2^{k p} .
\end{aligned}
$$

Now, choosing $\alpha_{(k, j)}=2^{-k} j^{-2}$, we obtain

$$
\begin{aligned}
\sum_{k_{0} \leq k \leq n} \sum_{j \leq 2^{[\beta k]}} \alpha_{(k, j)}^{-p} 2^{k p} & \leq \sum_{k_{0} \leq k \leq n} \sum_{j \leq 2^{[\beta k]}} 2^{2 k p} j^{2 p} \leq 2^{2 n p} \sum_{k_{0} \leq k \leq n} \sum_{j \leq 2^{[\beta k]}} j^{2 p} \\
& \leq 2^{2 n p} \sum_{k_{0} \leq k \leq n} 2^{(2 p+1)[\beta k]} \leq 2^{2 n p} 2^{(2 p+1)[\beta n]} n \leq 2^{n c_{\alpha, \beta}}
\end{aligned}
$$

which implies that

$$
R_{1} \leq c_{\varepsilon, \beta, \lambda_{1}, \lambda_{2}, \eta}\left(1+\lambda_{0}+\mu_{\delta}\right)^{p+\gamma} \exp \left(-\frac{1}{4} \gamma \lambda_{1} 2^{\varepsilon^{2} n / 2}\right) 2^{n c_{\alpha, \beta}} x^{-p}
$$

Since $\gamma=\frac{2 \eta}{p+2 \eta} \leq \eta \leq p \eta$ and $x \geq 1$, we conclude that

$$
R_{1} \leq A^{\prime} \exp \left(-\frac{1}{4} \gamma \lambda_{1}\left(2^{n}\right)^{\varepsilon^{2} / 2}\right)
$$


for some $A^{\prime}=c_{\varepsilon, \beta, \lambda_{1}, \lambda_{2}, \alpha, \alpha^{\prime}, \eta}^{\prime}\left(1+\lambda_{0}+\mu_{\delta}\right)^{p(1+\eta)}$. Now we give a control for $R_{2}$. Using Doob's inequality, for any $\lambda>2$,

$$
\begin{aligned}
R_{2} & \leq 2 \mathbb{P}\left(\sup _{k_{0} \leq k,(k, j) \in \mathcal{K}_{N}}\left|\sum_{k_{0} \leq l,(l, i) \preceq(k, j)} V_{(l, i)}^{\prime}\right| \geq x\right) \\
& \leq 2 x^{-\lambda} \mathbb{E}\left(\sum_{k_{0} \leq l,(l, i) \in \mathcal{K}_{N}}\left|V_{(l, i)}^{\prime}\right|\right)^{\lambda} .
\end{aligned}
$$

By Rosenthal's inequality

$$
\begin{aligned}
\left(\mathbb{E}\left(\sum_{k_{0} \leq l,(l, i) \in \mathcal{K}_{N}}\left|V_{(l, i)}^{\prime}\right|\right)^{\lambda}\right)^{1 / \lambda} \leq & c_{\lambda}\left(\sum_{k_{0} \leq l,(l, i) \in \mathcal{K}_{N}} \mathbb{E}\left(\left|V_{(l, i)}^{\prime}\right|^{2}\right)\right)^{1 / 2} \\
& +c_{\lambda}\left(\sum_{k_{0} \leq l,(l, i) \in \mathcal{K}_{N}} \mathbb{E}\left(\left|V_{(l, i)}^{\prime}\right|^{\lambda}\right)\right)^{1 / \lambda} \\
\leq & c_{\lambda}^{\prime}\left(2^{\beta n}\right)^{1 / 2} .
\end{aligned}
$$

From (6.3), (6.4) and (6.5) we obtain

$$
\begin{aligned}
& \mathbb{P}\left(\sup _{k_{0} \leq k,(k, j) \in \mathcal{K}_{N}}\left|\sum_{(l, i) \preceq(k, j)}\left(X_{(l, i)}^{\prime}-Y_{(l, i)}^{\prime}\right)\right| \geq 2 x\right) \\
\leq & A^{\prime} \exp \left(-\frac{1}{4} \gamma \lambda_{1}\left(2^{n}\right)^{\varepsilon^{2} / 2}\right)+c_{\lambda}\left(2^{\beta n}\right)^{\lambda / 2} x^{-\lambda} .
\end{aligned}
$$

Choosing $x=\frac{1}{2}\left(2^{n}\right)^{\frac{1}{2}-\rho}$, we find

$$
\begin{aligned}
& \mathbb{P}\left(\left(2^{n}\right)^{-\frac{1}{2}} \sup _{k_{0} \leq k,(k, j) \in \mathcal{K}_{N}}\left|\sum_{(l, i) \preceq(k, j)}\left(X_{(l, i)}^{\prime}-Y_{(l, i)}^{\prime}\right)\right| \geq\left(2^{n}\right)^{-\rho}\right) \\
\leq & A^{\prime} \exp \left(-\frac{1}{4} \gamma \lambda_{1}\left(2^{n}\right)^{\varepsilon^{2} / 2}\right)+c_{\lambda}\left(2^{n}\right)^{-\frac{1}{2} \lambda(1-\beta-2 \rho)} .
\end{aligned}
$$

It remains to handle the sequence $X_{(k, j)}$ for $k \leq k_{0}-1$. The construction of the $X_{(k, j)}^{\prime}$ for $k \leq$ $k_{0}-1$ can be performed in an arbitrary way such that the sequences $\left(X_{(k, j)}^{\prime}\right)_{(k, j) \preceq\left(k_{0}-1, m_{k_{0}-1}\right)}$ and $\left(Y_{(k, j)}^{\prime}\right)_{(k, j) \preceq\left(k_{0}-1, m_{k_{0}-1}\right)}$ are independent and $Y_{(k, j)}^{\prime} \stackrel{d}{=} X_{(k, j)}$ for any $(k, j) \preceq\left(k_{0}-1, m_{k_{0}-1}\right)$. Since the sequence $\left(X_{k}\right)_{k \geq 1}$ satisfies Condition C1, so does $\left(X_{(k, j)}^{\prime}\right)_{(k, j) \preceq\left(k_{0}-1, m_{k_{0}-1}\right)}$. Using the maximal inequality stated in Proposition 6 and noting that the cardinality of 
the set $\left\{(k, j):(k, j) \preceq\left(k_{0}-1, m_{k_{0}-1}\right)\right\}$ is less or equal to $2^{\beta k_{0}} \leq 2^{\varepsilon n}$, we obtain, for any $\eta^{\prime} \in\left(0, \frac{\delta-\alpha}{(2+\alpha+\delta)^{2}}\right)$,

$$
\mathbb{E}\left(\sup _{(k, j) \preceq\left(k_{0}-1, m_{k_{0}-1}\right)}\left|\sum_{(l, i) \preceq(k, j)} X_{(l, i)}^{\prime}\right|^{p}\right) \leq A^{\prime \prime}\left(2^{\varepsilon n}\right)^{\frac{1}{2} p},
$$

for come constant $A^{\prime \prime}=c_{\varepsilon, \beta, \lambda_{1}, \lambda_{2}, \delta, \alpha, \eta}^{\prime \prime}\left(1+\lambda_{0}+\mu_{\delta}\right)^{p\left(1+\eta^{\prime}\right)}$. By Chebyshev's inequality, for any $x>0$ we get

$$
\begin{aligned}
\mathbb{P}\left(\sup _{(k, j) \preceq\left(k_{0}-1, m_{k_{0}-1}\right)}\left|\sum_{(l, i) \preceq(k, j)} X_{(l, i)}^{\prime}\right| \geq x\right) & \leq x^{-p} \mathbb{E} \sup _{(k, j) \preceq\left(k_{0}-1, m_{k_{0}-1}\right)}\left|\sum_{(l, i) \preceq(k, j)} X_{(l, i)}^{\prime}\right|^{p} \\
& \leq A^{\prime \prime} x^{-p}\left(2^{\varepsilon n}\right)^{\frac{1}{2} p} .
\end{aligned}
$$

Substituting $x=\left(2^{n}\right)^{\frac{1}{2}-\rho}$ yields

$$
\mathbb{P}\left(\left(2^{n}\right)^{-\frac{1}{2}} \sup _{(k, j) \preceq\left(k_{0}-1, m_{k_{0}-1}\right)}\left|\sum_{(l, i) \preceq(k, j)} X_{(l, i)}^{\prime}\right| \geq\left(2^{n}\right)^{-\rho}\right) \leq A^{\prime \prime}\left(2^{n}\right)^{-\frac{p}{2}+p\left(\rho+\frac{1}{2} \varepsilon\right)} .
$$

A similar inequality can be proved with $Y_{(l, i)}^{\prime}$ instead of $X_{(l, i)}^{\prime}$. Combining this with (6.6) we obtain,

$$
\begin{aligned}
& \mathbb{P}\left(\left(2^{n}\right)^{-\frac{1}{2}} \sup _{(k, j) \in \mathcal{K}_{N}}\left|\sum_{(l, i) \preceq(k, j)}\left(X_{(l, i)}^{\prime}-Y_{(l, i)}^{\prime}\right)\right| \geq 2\left(2^{n}\right)^{-\rho}\right) \\
\leq & A^{\prime \prime \prime}\left(\exp \left(-\frac{1}{4} \gamma \lambda_{1}\left(2^{n}\right)^{\varepsilon^{2} / 2}\right)+\left(2^{n}\right)^{-\frac{1}{2} \lambda(1-\beta-2 \rho)}+\left(2^{n}\right)^{-\frac{p}{2}+p\left(\rho+\frac{1}{2} \varepsilon\right)}\right),
\end{aligned}
$$

for some $A^{\prime \prime}=c_{\varepsilon, \beta, \lambda_{1}, \lambda_{2}, \delta, \delta^{\prime}, \eta, \lambda}^{\prime \prime \prime}\left(1+\lambda_{0}+\mu_{\delta}\right)^{p\left(1+\eta+\eta^{\prime}\right)}$. Recall that $p=2+2 \alpha, \alpha<\delta, \beta>\frac{1}{2}$ and $\rho<\frac{1-\beta}{2}$. Taking $\lambda=\frac{2+2 \alpha}{1-\beta-2 \rho}>p$, the right-hand side of (6.7) does not exceed $A^{\prime \prime \prime}\left(2^{n}\right)^{-1-\alpha+(2+2 \alpha)(\rho+\varepsilon)}$. It remains to choose a sufficiently small $\eta+\eta^{\prime}$ such that $p\left(1+\eta+\eta^{\prime}\right) \leq$ $2+2 \delta$, which implies

$$
A^{\prime \prime \prime} \leq c_{\varepsilon, \beta, \lambda_{1}, \lambda_{2}, \alpha, \rho}^{\prime \prime \prime}\left(1+\lambda_{0}+\mu_{\delta}\right)^{2+2 \delta} .
$$

The assertion of the proposition follows.

6.2. Coupling with independent normal r.v.'s. We assume that $\mu_{i}=0, i \geq 1$ and $\sigma^{2}>$ 0 . The following proposition shows that with high probability the partial sums $\sum_{(l, i) \preceq(k, j)} X_{(l, i)}$ can be coupled with the partial sums of some normal r.v.'s. Denote $\sigma_{l, i}^{2}=\operatorname{Var}\left(X_{(l, i)}\right)$.

Proposition 5. Assume conditions $\boldsymbol{C 1}, \boldsymbol{C} 2$ and $\boldsymbol{C 3}$ with $\mu_{i}=0, i \geq 1$ and $\sigma=1$. Let $\alpha<\delta$, $\beta>\frac{1}{2}$ and $0<\rho<\frac{1-\beta}{2}$. Then for any $N \in \mathbb{N}$, on some extension of the initial probability 
space there exists a sequence of independent standard normal r.v.'s $\left(W_{(k, j)}^{\prime}\right)_{(k, j) \in \mathcal{K}_{N}}$ and a version $\left(X_{(k, j)}^{\prime}\right)_{(k, j) \in \mathcal{K}_{N}}$ of the sequence $\left(X_{(k, j)}\right)_{(k, j) \in \mathcal{K}_{N}}$ such that

$$
\begin{aligned}
& \mathbb{P}\left(\left(2^{n}\right)^{-\frac{1}{2}} \sup _{(k, j) \in \mathcal{K}_{N}}\left|\sum_{(l, i) \preceq(k, j)}\left(X_{(l, i)}^{\prime}-\sigma_{l, i} W_{(l, i)}^{\prime}\right)\right| \geq 2\left(2^{n}\right)^{-\rho}\right) \\
\leq & C_{2}\left(2^{n}\right)^{-1-\alpha+(\varepsilon+\rho)(2+2 \alpha)} \quad \text { (dependence error) } \\
& +C_{2}\left(2^{n}\right)^{-\beta \alpha+\rho(2+2 \alpha)} \quad \text { (Sakhanenko's error) }
\end{aligned}
$$

where $C_{2}=c_{\varepsilon, \beta, \lambda_{1}, \lambda_{2}, \alpha, \rho}\left(1+\lambda_{0}+\mu_{\delta}\right)^{2+2 \delta}$ and $c_{\varepsilon, \beta, \lambda_{1}, \lambda_{2}, \alpha, \rho}$ is a positive constant depending only on its indices.

Proof. Let $p=2+2 \alpha$. Since $\left|I_{k, j}\right| \leq 2^{k-[\beta k]}$, we obtain, using Proposition 7

$$
\mathbb{E}\left|X_{(k, j)}\right|^{p} \leq A\left|I_{k, j}\right|^{\frac{p}{2}} \leq A\left(2^{k-[\beta k]}\right)^{\frac{p}{2}}
$$

where $A=c_{\lambda_{1}, \lambda_{2}, \delta^{\prime}, \eta}\left(1+\lambda_{0}+\mu_{\delta}\right)^{p(1+\eta)}$ and $\eta>0$ is arbitrary. Taking into account that $m_{k}=2^{[\beta k]} \leq 2^{\beta k}$, we have

$$
\begin{aligned}
\sum_{(k, j) \in \mathcal{K}_{N}} \mathbb{E}\left|X_{(k, j)}\right|^{p} & =\sum_{k=1}^{n} \sum_{j=1}^{m_{k}} \mathbb{E}\left|X_{(k, j)}\right|^{p} \\
& \leq \sum_{k=1}^{n} m_{k} A\left(2^{k-[\beta k]}\right)^{\frac{p}{2}} \\
& \leq A 2^{\frac{p}{2}} \sum_{k=1}^{n} 2^{k\left(\beta+\frac{p}{2}(1-\beta)\right)} \\
& \leq c_{\alpha, \beta} A\left(2^{n}\right)^{\beta+\frac{p}{2}(1-\beta)}
\end{aligned}
$$

By (7.2) in Section 7.2, on some probability space $\left(\Omega^{\prime \prime}, \mathcal{F}^{\prime \prime}, \mathbb{P}^{\prime \prime}\right)$, there exist a version $\left(Y_{(k, j)}^{\prime \prime}\right)_{(k, j) \in \mathcal{K}_{N}}$ of the sequence $\left(Y_{(k, j)}\right)_{(k, j) \in \mathcal{K}_{N}}$ and independent standard normal r.v.'s $\left(W_{(k, j)}^{\prime}\right)_{(k, j) \in \mathcal{K}_{N}}$ such that

$$
\mathbb{P}^{\prime \prime}\left(\sup _{(k, j) \in \mathcal{K}_{N}^{0}}\left|\sum_{(l, i) \preceq(k, j)}\left(Y_{(l, i)}^{\prime \prime}-\sigma_{l, i} W_{(l, i)}^{\prime}\right)\right| \geq a\right) \leq \frac{c_{p}}{a^{p}} \sum_{(k, j) \in \mathcal{K}_{N}^{0}} \mathbb{E}\left|X_{(k, j)}\right|^{p}
$$

Choosing $a=\left(2^{\beta n}\right)^{\frac{1}{2}-\rho}$ and taking into account (6.9) we obtain

$$
\begin{aligned}
& \mathbb{P}^{\prime \prime}\left(\left(2^{n}\right)^{-\frac{1}{2}} \sup _{(k, j) \in \mathcal{K}_{N}^{0}}\left|\sum_{(l, i) \preceq(k, j)}\left(Y_{(l, i)}^{\prime \prime}-\sigma_{l, i} W_{(l, i)}^{\prime}\right)\right| \geq\left(2^{n}\right)^{-\rho}\right) \\
\leq & c_{p}\left(2^{n}\right)^{-\frac{p}{2}+\rho p} c_{\alpha, \beta} A\left(2^{n}\right)^{\beta+\frac{p}{2}(1-\beta)} \\
\leq & c_{\alpha, \beta}^{\prime} A\left(2^{n}\right)^{-\beta \alpha+\rho(2+2 \alpha)} .
\end{aligned}
$$


By Berkes-Philip's lemma [1] we can reconstruct the sequences $\left(X_{(k, j)}^{\prime}\right)_{(k, j) \in \mathcal{K}_{N}},\left(Y_{(k, j)}^{\prime}\right)_{(k, j) \in \mathcal{K}_{N}}$, $\left(Y_{(k, j)}^{\prime \prime}\right)_{(k, j) \in \mathcal{K}_{N}}$ and $\left(W_{(k, j)}^{\prime}\right)_{(k, j) \in \mathcal{K}_{N}}$ on some new probability space in such a way that a.s.

$$
Y_{(k, j)}^{\prime}=Y_{(k, j)}^{\prime \prime},(k, j) \in \mathcal{K}_{N}
$$

Without loss of generality we shall consider this new probability space as an extension of the initial probability space. Using Proposition 4 we obtain

$$
\begin{aligned}
& \mathbb{P}\left(2^{-\frac{n}{2}} \sup _{(k, j) \in \mathcal{K}_{N}}\left|\sum_{(l, i) \preceq(k, j)}\left(X_{(l, i)}^{\prime}-\sigma_{l, i} W_{(l, i)}^{\prime}\right)\right| \geq 2\left(2^{n}\right)^{-\rho}\right) \\
\leq & C_{1}\left(2^{n}\right)^{-1-\alpha+(\varepsilon+\rho)(2+2 \alpha)} \quad \text { (dependence error) } \\
& +c_{\delta, \beta}^{\prime} A\left(2^{n}\right)^{-\beta \alpha+\rho(2+2 \alpha)} \quad \text { (Sakhanenko's error) }
\end{aligned}
$$

with $C_{1}$ defined by Proposition 4 . Taking into account that $p=2+2 \alpha, \alpha<\delta$ and choosing $\eta$ sufficiently small we get $p(1+\eta) \leq 2+2 \delta$, which implies $c_{\alpha, \beta}^{\prime} A \leq c_{\lambda_{1}, \lambda_{2}, \alpha, \eta}^{\prime}\left(1+\lambda_{0}+\mu_{\delta}\right)^{2+2 \delta}$. This concludes the proof.

6.3. Construction of the sequences $\left(\widetilde{X}_{i}\right)_{1 \leq i \leq N}$ and $\left(W_{i}\right)_{1 \leq i \leq N}$. As before, we suppose that $\mu_{i}=0, i \geq 1$ and $\sigma^{2}=1$. Let $\left(X_{(k, j)}^{\prime}\right)_{(k, j) \in \mathcal{K}_{N}}$ and $\left(W_{(k, j)}^{\prime}\right)_{(k, j) \in \mathcal{K}_{N}}$ be the sequences which appear in Proposition 5.

First we shall construct the sequence $\left(W_{i}\right)_{1<i<N}$. Note that, by condition C3, the variances $\sigma_{k, i}^{2}=\operatorname{Var}\left(X_{(k, j)}\right)$ can be approximated by $\sigma^{2}\left|I_{k, j}\right|=\left|I_{k, j}\right|$, but in general do not coincide with $\left|I_{k, j}\right|$. Therefore to perform our construction we have to replace each of the non identically distributed normal random variables $\sigma_{k, j}^{2} W_{(k, j)}^{\prime}$ by some sums of independent identically distributed standart normal random variables. Let $\left(W_{i}\right)_{1<i<N}$ be a sequence of independent standart normal r.v.'s; let $\xi_{k, j}$ be an extra standart normal random variable. Set $I_{k, j}:=\left\{i_{1}, \cdots, i_{\left|I_{k, j}\right|}\right\}$ with $i_{1} \preceq \ldots \preceq i_{\left|I_{k, j}\right|}$ and let $i_{k, j}^{*}$ be the maximal index $j \in\left\{i_{1}, \ldots, i_{\left|I_{k, j}\right|}\right\}$ for which the variance of the partial sum $\sum_{i=i_{1}}^{j} W_{i}$ does not exceed $\sigma_{k, j}^{2}$, i.e. $i_{k, j}^{*}=i_{m_{k, j}^{*}}$, where $m_{k, j}^{*}=\min \left\{\left|I_{k, j}\right|,\left[\sigma_{k, j}^{2}\right]\right\}$. Set $W_{(k, j)}^{\prime \prime}=\sum_{i=i_{1}}^{i_{k, j}^{*}} W_{i}+\xi_{k, j} f_{k, j}$ where $f_{k, j}^{2}=\left|\sigma_{k, j}^{2}-i_{k, j}^{*}\right|$. It is easy to verify that $W_{(k, j)}^{\prime \prime}$ is a normal random variable of mean 0 and variance $\sigma_{k, j}^{2}$ and that $\sum_{k \in I_{k, j}} W_{k}-W_{(k, j)}^{\prime \prime}=\sum_{i_{k, j}^{*}+1 \leq k \leq\left|I_{k, j}\right|} W_{k}-\xi_{k, j} f_{k, j}$ is a normal random variable of mean 0 and variance $\left(\left|I_{k, j}\right|-i_{k, j}^{*}\right)+f_{k, j}^{2}=\left|\sigma_{k, j}^{2}-\right| I_{k, j}||$. We consider the random variable $W_{(k, j)}^{\prime \prime}$ as a new version of $\sigma_{k, j} W_{(k, j)}^{\prime}$. By Berkes-Philip's lemma [1], without loss of generality, we can reconstruct the sequences $\left(X_{(k, j)}^{\prime}\right)_{(k, j) \in \mathcal{K}_{N}}$, $\left(\sigma_{k, j} W_{(k, j)}^{\prime}\right)_{(k, j) \in \mathcal{K}_{N}},\left(W_{(k, j)}^{\prime \prime}\right)_{(k, j) \in \mathcal{K}_{N}}$ and $\left(W_{(k, j)}^{\prime \prime}\right)_{(k, j) \in \mathcal{K}_{N}}$ on the same probability space such that a.s. $\left(\sigma_{k, j} W_{(k, j)}^{\prime}\right)_{(k, j) \in \mathcal{K}_{N}}=\left(W_{(k, j)}^{\prime \prime}\right)_{(k, j) \in \mathcal{K}_{N}}$. We shall consider that this probability space is an extension of the initial probability space. Thus we have constructed the $W_{i}$ 's when $i$ belongs to the union of all islands $I=\cup_{(k, j) \in \mathcal{K}_{N}} I_{k, j}$ with the property that the $\eta_{k, j}=\sum_{i \in I_{k, j}} W_{i}-\sigma_{k, j} W_{(k, j)}^{\prime}$ are independent normal and centered random variables with 
variances $v_{k, j}^{2}=\left|\sigma_{k, j}^{2}-\right| I_{k, j}|| \leq \tau\left|I_{k, j}\right|^{\gamma}$, for any $\gamma>0$. Therefore the sum $\sum_{(l, i) \leq(n, m)} \eta_{l, i}$ is normal with mean 0 and variance $\sum_{(l, i) \leq(n, m)} v_{k, i}^{2} \leq c_{\beta} \tau 2^{(\beta+\gamma) n}$; by Doob's inequality, with $p=2+2 \alpha$, it follows

$$
\begin{aligned}
\mathbb{P}\left(\left(2^{n}\right)^{-\frac{1}{2}} \sup _{(k, j) \in \mathcal{K}_{N}}\left|\sum_{(l, i) \leq(k, j)} \eta_{l, i}\right| \geq\left(2^{n}\right)^{-\rho}\right) & \leq\left(2^{n}\right)^{-p / 2+\rho p} \mathbb{E}\left(\left|\sum_{(l, i) \leq(n, m)} \eta_{l, i}\right|^{p}\right) \\
& \leq c_{\alpha, \beta}\left(2^{n}\right)^{-p / 2+\rho p}\left(\tau 2^{(\beta+\gamma) n}\right)^{p / 2} \\
& =c_{\alpha, \beta} \tau^{1+\alpha}\left(2^{n}\right)^{-(1-\beta)(1+\alpha)+(\rho+\gamma / 2)(2+2 \alpha)},
\end{aligned}
$$

where $\gamma>0$ is arbitrary. When $i$ belongs to the union of gaps $J=\cup_{(k, j) \in \mathcal{K}_{N}} J_{k, j}$ the variables $W_{i}$ can be taken as any independent standard normal random variables independent of the sequence $\left(W_{k}\right)_{k \in I}$.

To extend the construction of the sequence $\left(X_{(k, j)}^{\prime}\right)_{(k, j) \in \mathcal{K}_{N}}$ to the sequence $\left(\widetilde{X}_{i}\right)_{1 \leq i \leq N}$ we have to construct, for each $(k, j) \in \mathcal{K}_{N}$, a sequence $\left(\widetilde{X}_{i}\right)_{i \in I_{k, j}}$ such that $\sum_{i \in I_{k, j}} \widetilde{X}_{i}=X_{(k, j)}^{\prime}$ and $\left(\widetilde{X}_{i}\right)_{i=1, \ldots, N} \stackrel{d}{=}\left(X_{i}\right)_{i=1, \ldots, N}$. Denote by $F_{X \mid Y_{1}, \ldots, Y_{k}}\left(x \mid y_{1}, \ldots, y_{k}\right)$ the conditional distribution of $X$ given $\left[Y_{1}=y_{1}, \ldots, Y_{k}=y_{k}\right]$. Without loss of generality, on the initial probability space, there exists a sequence $U_{1}, \ldots, U_{N}$ of independent r.v.'s uniformly distributed on $(0,1)$. Let $i_{1}, \ldots, i_{\left|I_{k, j}\right|}$ be the indices in the set $I_{k, j}$. The required construction is performed in the standard way by defining first $\widetilde{X}_{i_{1}}$ as the conditional quantile transform $F_{X_{i_{1}} \mid X_{(k, j)}}^{-1}\left(U_{k, i_{1}} \mid X_{(k, j)}^{\prime}\right)$ and then by setting $\tilde{X}_{i_{l}}=F_{X_{i_{l}} \mid X_{i_{1}}, \ldots, X_{i_{l-1}}, X_{(k, j)}}^{-1}\left(U_{i_{l}} \mid \tilde{X}_{i_{1}}, \ldots, \tilde{X}_{i_{l-1}}, X_{(k, j)}^{\prime}\right)$, for $l=2, \ldots,\left|I_{k, j}\right|$. Thus we have constructed the vector $\widetilde{X}_{I}=\left(\widetilde{X}_{i}\right)_{i \in I}$, where $I=\cup_{(k, j) \in \mathcal{K}_{N}} I_{k, j}$ is the union of all islands such that $\widetilde{X}_{I} \stackrel{d}{=} X_{I}=\left(X_{i}\right)_{i \in I}$. In the same way we construct the $\widetilde{X}_{i}$ when $i$ belongs to the union of gaps $J=\cup_{(k, j) \in \mathcal{K}_{N}} J_{k, j}:=\left\{j_{1}, \ldots, j_{|J|}\right\}$ : set $X_{j_{1}}=F_{X_{j_{1}} \mid X_{I}}^{-1}\left(U_{k, j_{1}} \mid \widetilde{X}_{I}\right)$ and subsequently $\widetilde{X}_{j_{l}}=F_{X_{j_{l}} \mid X_{j_{1}}, \ldots, X_{j_{l-1}}, X_{I}}^{-1}\left(U_{j_{l}} \mid \widetilde{X}_{j_{1}}, \ldots, \widetilde{X}_{j_{l-1}}, \widetilde{X}_{I}\right)$, for $l=2, \ldots,|J|$.

6.4. Putting together the bounds. Denote by $r_{k, j}$ the right end of the island $I_{k, j}$ and let $\mathcal{L}_{N}=\{1\} \cup\left\{r_{k, j}:(k, j) \in \mathcal{K}_{N}\right\}$ be the set of $r_{k, j}$, ordered with respect to lexicographical order $\preceq$. For any $r=r_{k, j} \in \mathcal{L}_{N}$ denote by $r^{\text {next }}$ be the next element in the set $\mathcal{L}_{N}$, i.e. $r^{\text {next }}=\inf \left\{r^{\prime}: r^{\prime} \in \mathcal{L}_{N}, r \preceq r^{\prime}\right\}$.

Let $\left(\widetilde{X}_{i}\right)_{1 \leq i \leq N}$ and $\left(W_{i}\right)_{1 \leq i \leq N}$ be the sequences constructed in Section 6.3. Recall that by construction, for any $r=r_{k, j} \in \mathcal{L}_{N}$, we have $\{1, \ldots, r\}=\sum_{(l, i) \preceq(k, j)} J_{l, j} \cup I_{k, j}$. First we replace in the statement of Theorem 1 the sup over the set $\{j: 1 \leq j \leq N\}$ by the sup over the grid $\mathcal{L}_{N}$ and the sup of the oscillation term: in other words, $\sup _{1 \leq j \leq N}\left|\sum_{i \leq j}\left(\widetilde{X}_{i}-W_{i}\right)\right|$ is 
bounded by

$$
\underbrace{\sup _{r \in \mathcal{L}_{N}}\left|\sum_{i \leq r}\left(\tilde{X}_{i}-W_{i}\right)\right|}_{\text {(sup over the grid } \left.\mathcal{L}_{N}\right)}+\underbrace{\sup _{r \in \mathcal{L}_{N}} \sup _{r \leq r^{\prime} \leq \min \left\{r^{n e x t}-1, N\right\}}\left|\sum_{r \leq i \leq r^{\prime}}\left(\tilde{X}_{i}-W_{i}\right)\right|}_{\text {(oscillation term) }} .
$$

For any $r=r_{k, j}$, we have

$$
\sum_{1 \leq i \leq r} \tilde{X}_{i}=\sum_{(l, h) \preceq(k, j)}\left(\sum_{i \in I_{l, h}} \tilde{X}_{i}+\sum_{i \in J_{l, h}} \tilde{X}_{i}\right)=\sum_{(l, h) \preceq(k, j)}\left(\underline{X}_{(l, h)}+\bar{X}_{(l, h)}\right),
$$

where $\underline{X}_{(l, h)}=\sum_{i \in I_{l, h}} \tilde{X}_{i}$ and $\bar{X}_{(l, h)}=\sum_{i \in J_{l, h}} \tilde{X}_{i}$. In the same way

$$
\sum_{1 \leq i \leq r} W_{i}=\sum_{(l, h) \preceq(k, j)}\left(\sum_{i \in I_{l, h}} W_{i}+\sum_{i \in J_{l, h}} W_{i}\right)=\sum_{(l, h) \preceq(k, j)}\left(\underline{W}_{(l, h)}+\bar{W}_{(l, h)}\right)
$$

where $\underline{W}_{(l, h)}=\sum_{i \in I_{l, h}} W_{i}$ and $\bar{W}_{(l, h)}=\sum_{i \in J_{l, h}} W_{i}$. From (6.11), (6.12) and (6.13) we obtain

$$
\begin{aligned}
& \sup _{1 \leq j \leq N}\left|\sum_{i \leq j}\left(\widetilde{X}_{i}-W_{i}\right)\right| \\
\leq & \sup _{(k, j) \in \mathcal{K}_{N}}\left|\sum_{(l, h) \leq(k, j)} \underline{X}_{(l, h)}-\underline{W}_{(l, h)}\right| \quad \text { (sup over islands) } \\
+ & \sup _{(k, j) \in \mathcal{K}_{N}}\left|\sum_{(l, h) \leq(k, j)}\left(\bar{X}_{(l, h)}-\bar{W}_{(l, h)}\right)\right| \quad \text { (sup over gaps) } \\
& +\sup _{r \in \mathcal{L}_{N}} \sup _{r \leq r^{\prime} \leq \min \left\{r^{\text {next }}-1, N\right\}}\left|\sum_{l \leq i \leq r^{\prime}}\left(\widetilde{X}_{i}-W_{i}\right)\right| \quad \text { (oscillation term) }
\end{aligned}
$$

where the term "sup over islands" is bounded by the sum

$$
\begin{aligned}
& \sup _{(k, j) \in \mathcal{K}_{N}}\left|\sum_{(l, h) \leq(k, j)} \underline{X}_{(l, h)}-\sigma_{l, h} W_{(l, h)}^{\prime}\right| \quad \text { (normal approximation) } \\
& +\sup _{(k, j) \in \mathcal{K}_{N}}\left|\sum_{(l, h) \leq(k, j)}\left(\sigma_{l, h} W_{(l, h)}^{\prime}-\underline{W}_{(l, h)}\right)\right| \cdot \quad \text { (variance homogenization) }
\end{aligned}
$$

The control of the term "normal approximation" has been yet given in Proposition 5 where it is bounded by two terms "dependence error" and "Sakhanenko's error". The "variance homogenization" term is controlled by (6.10). As to terms "sup over gaps" and "oscillation term" they will be considered in (6.17) and (6.18) below. 
6.5. Bound for the partial sums over gaps. Let $p=2+2 \alpha$, where $\alpha<\delta$. Since the blocks are indexed by $l=k_{0}, \ldots, n$ and the total length of the gaps in the block $l$ is less than $(2+[\beta l]) 2^{[\beta l]+[\varepsilon l]-1}$ the total length $L^{g a p}$ of all gaps satisfies

$$
L^{\text {gap }}=\sum_{(l, i) \preceq(n, m)} \sum_{h \in J_{l, i}}=\sum_{k_{0} \leq l \leq n}(2+[\beta l]) 2^{[\beta l]+[\varepsilon l]-1} \leq c_{\varepsilon, \beta} 2^{(\beta+\varepsilon) k} .
$$

By Proposition 6, we have, for any $\eta>0$,

$$
\begin{aligned}
\left\|\sup _{(k, j) \preceq(n, m)} \mid \sum_{(l, i) \leq(k, j)} \bar{X}_{(l, i)}\right\| \|_{L^{p}} & \leq c_{\lambda_{1}, \lambda_{2}, \alpha, \delta, \eta}\left(1+\lambda_{0}+\mu_{\delta}\right)^{1+\eta}\left(\sum_{(l, h) \preceq(n, m)} \sum_{h \in J_{l, i}}\right)^{\frac{1}{2}} \\
& \leq c_{\varepsilon, \beta, \lambda_{1}, \lambda_{2}, \alpha, \delta, \eta}\left(1+\lambda_{0}+\mu_{\delta}\right)^{1+\eta}\left(2^{(\beta+\varepsilon) n}\right)^{\frac{1}{2}}
\end{aligned}
$$

where $c_{\varepsilon, \beta, \lambda_{1}, \lambda_{2}, \alpha, \delta, \eta}$ is a constant depending only on its indices. Using Chebyshev's inequality with $x=\left(2^{n}\right)^{\frac{1}{2}-\rho}$, we get

$$
\begin{aligned}
\mathbb{P}\left(\sup _{(k, j) \in \mathcal{K}_{N}}\left|\sum_{(l, i) \leq(k, j)} \bar{X}_{(l, i)}\right| \geq x\right) & \leq \frac{1}{x^{p}} \mathbb{E}\left(\sup _{(k, j) \preceq(n, m)}\left|\sum_{(l, i) \leq(k, j)} \bar{X}_{(l, i)}\right|\right)^{p} \\
& \leq A\left(2^{n}\right)^{-p / 2+\rho p}\left(2^{(\beta+\varepsilon) n}\right)^{p / 2} \\
& \leq A\left(2^{n}\right)^{-(1-\beta-\varepsilon) p / 2+\rho p}
\end{aligned}
$$

where $A=c_{\varepsilon, \beta, \lambda_{1}, \lambda_{2}, \alpha, \delta, \eta}\left(1+\lambda_{0}+\mu_{\delta}\right)^{p(1+\eta)}$. A similar bound can be established with $\bar{W}_{(l, i)}$ instead of $\bar{X}_{(l, i)}$. Using this bound and (6.16) it follows that, for any $0<\rho<\frac{1}{4}$,

$$
\begin{aligned}
& \mathbb{P}\left(\left(2^{n}\right)^{-\frac{1}{2}} \sup _{(k, j) \in \mathcal{K}_{N}}\left|\sum_{(l, i) \leq(k, j)}\left(\bar{X}_{(l, i)}-\bar{W}_{(l, i)}\right)\right| \geq\left(2^{n}\right)^{-\rho}\right) \\
\leq & A^{\prime}\left(2^{n}\right)^{-(1-\beta-\varepsilon) p / 2+\rho p} \\
\leq & A^{\prime}\left(2^{n}\right)^{-(1-\beta)(1+\alpha)+(\rho+\varepsilon / 2)(2+2 \alpha)}
\end{aligned}
$$

where $A^{\prime}=c_{\varepsilon, \beta, \lambda_{1}, \lambda_{2}, \alpha, \delta}^{\prime}\left(1+\lambda_{0}+\mu_{\delta}\right)^{2+2 \delta}$.

6.6. Bound for the oscillation term. Denote for brevity $r^{+}=\min \left\{r^{\text {next }}-1, N\right\}$. First note that

$$
r^{+}-r \leq \max _{(k, j) \in \mathcal{K}_{N}}\left(\left|I_{k, j}\right|+\left|J_{k, j}\right|\right) \leq c_{\varepsilon, \beta}\left(2^{(\beta+\varepsilon) n}+2^{(1-\beta) n}\right) .
$$

Let $p=2+2 \alpha$ where $\alpha<\delta$. By Proposition 6 of Section 8 , for any $\eta>0$, we have

$$
\left\|\sup _{r \in \mathcal{L}_{N}} \sup _{r \leq l \leq r^{+}}\left|\sum_{r \leq i \leq l} \widetilde{X}_{i}\right|\right\|_{L^{p}}^{p} \leq A\left(\sup _{r \in \mathcal{L}_{N}}\left(r^{+}-r\right)\right)^{p / 2} \leq c_{\varepsilon, \beta} A\left(2^{(\beta+\varepsilon) n}+2^{(1-\beta) n}\right)^{p / 2},
$$


where $A=c_{\lambda_{1}, \lambda_{2}, \alpha, \delta, \eta}\left(1+\lambda_{0}+\mu_{\delta}\right)^{p(1+\eta)}$. Therefore, by Chebyshev's inequality, with $x=$ $\frac{1}{2}\left(2^{n}\right)^{\frac{1}{2}-\rho}$ and $\rho>0$,

$$
\begin{aligned}
\mathbb{P}\left(\sup _{r \in \mathcal{L}_{N}} \sup _{r \leq l \leq r^{+}}\left|\sum_{r \leq i \leq l} \widetilde{X}_{i}\right| \geq x\right) & \leq x^{-p} \mathbb{E}\left(\sup _{r \in \mathcal{L}_{N}} \sup _{r \leq l \leq r^{+}}\left|\sum_{r \leq i \leq l} \widetilde{X}_{i}\right|\right)^{p} \\
& \leq c_{\varepsilon, \beta} A x^{-p}\left(2^{(\beta+\varepsilon) n}+2^{(1-\beta) n}\right)^{p / 2} \\
& \leq c_{\varepsilon, \beta}^{\prime} A 2^{p+n p \rho}\left(\left(2^{n}\right)^{-(1+\alpha)(1-\beta-\varepsilon)}+\left(2^{n}\right)^{-(1+\alpha) \beta}\right) .
\end{aligned}
$$

Choosing $\eta$ small enough we have $p(1+\eta) \leq 2+2 \delta$ and therefore

$$
c_{\varepsilon, \beta} 2^{p} A \leq A^{\prime}=c_{\varepsilon, \beta, \lambda_{1}, \lambda_{2}, \alpha, \delta}^{\prime}\left(1+\lambda_{0}+\mu_{\delta}\right)^{2+2 \delta} .
$$

Since a similar bound can be established with $W_{i}$ instead of $X_{i}$, we obtain the following bound for the oscilation term:

$$
\begin{aligned}
& \mathbb{P}\left(\left(2^{n}\right)^{-\frac{1}{2}} \sup _{r \in \mathcal{L}_{N}} \sup _{r \leq l \leq r^{+}}\left|\sum_{r \leq i \leq l}\left(\widetilde{X}_{i}-W_{i}\right)\right| \geq 2\left(2^{n}\right)^{-\rho}\right) \\
\leq & 2 A^{\prime}\left(2^{n}\right)^{(2+2 \alpha)(\rho+\varepsilon / 2)}\left(\left(2^{n}\right)^{-(1+\alpha)(1-\beta)}+\left(2^{n}\right)^{-(1+\alpha) \beta}\right) .
\end{aligned}
$$

6.7. Optimizing the bounds. Let $\alpha<\delta, \beta>\frac{1}{2}$ and $0<\rho<\frac{1-\beta}{2}$. Using (6.14)), we may decompose $\mathbb{P}\left(\left(2^{n}\right)^{-\frac{1}{2}} \sup _{1 \leq j \leq N}\left|\sum_{i=1}^{j}\left(\widetilde{X}_{i}-W_{i}\right)\right| \geq 6\left(2^{n}\right)^{-\rho}\right)$ in 3 terms, the first one "sup over islands" being itself decomposed in two terms (see (6.15)); consequently, this quantity is decomposed in four terms listed below:

- the first term "normal approximation" is controlled with Proposition 5, it is bounded by two terms named "dependence error" and "Sakhanenko's error",

- the second term "variance homogenization" is controlled in 6.10 with $\gamma=\varepsilon$

- the term "sup over gaps" is controlled in (6.17),

- the term "oscillation term" is controlled in (6.18).

Putting these bounds altogether, we obtain

$$
\begin{aligned}
& \mathbb{P}\left(\left(2^{n}\right)^{-\frac{1}{2}} \sup _{1 \leq j \leq N}\left|\sum_{i=1}^{j}\left(X_{i}-W_{i}\right)\right| \geq 6\left(2^{n}\right)^{-\rho}\right) \\
& \leq A\left(2^{n}\right)^{-(1+\alpha)+(\rho+\varepsilon)(2+2 \alpha)} \quad \text { (dependence error) } \\
& +A\left(2^{n}\right)^{-\beta \alpha+\rho(2+2 \alpha)} \quad \text { (Sakhanenko's error) } \\
& +A\left(2^{n}\right)^{-(1-\beta)(1+\alpha)+(\rho+\varepsilon / 2)(2+2 \alpha)} \quad \text { (variance homogenization error) } \\
& +A\left(2^{n}\right)^{-(1-\beta)(1+\alpha)+(\rho+\varepsilon / 2)(2+2 \alpha)} \quad \text { (gaps error) } \\
& +A\left(\left(2^{n}\right)^{-(1-\beta)(1+\alpha)}+\left(2^{n}\right)^{-\beta(1+\alpha)}\right)\left(2^{n}\right)^{(\rho+\varepsilon / 2)(2+2 \alpha)} \quad \text { (oscillation error) }
\end{aligned}
$$


where $A=c_{\varepsilon, \beta, \lambda_{1}, \lambda_{2}, \alpha}\left(1+\tau^{1+\alpha}+\left(1+\lambda_{0}+\mu_{\delta}\right)^{2+2 \alpha}\right)$ and $c_{\varepsilon, \beta, \lambda_{1}, \lambda_{2}, \alpha, \delta}$ is a positive constant depending only on its indices. For the moment let us ignore the factors containing $\varepsilon$ which have a small contribution to the bound.

The term (dependence error) is negligible with respect to all other terms; equating the powers of the term (Sakhanenko's error) and the term (gaps error) (or equivalently variance homogenization error) we get $\beta \alpha=(1-\beta)(1+\alpha)$ i.e. $\beta=\frac{1+\alpha}{1+2 \alpha}$. Implementing $\beta=\frac{1+\alpha}{1+2 \alpha}$ in the above inequality yields

$$
\begin{gathered}
\mathbb{P}\left(\left(2^{n}\right)^{-\frac{1}{2}} \sup _{1 \leq l \leq N}\left|\sum_{i=1}^{l}\left(\widetilde{X}_{i}-W_{i}\right)\right| \geq 6\left(2^{n}\right)^{-\rho}\right) \\
\leq A\left(2^{n}\right)^{-1-\delta+(\rho+\varepsilon)(2+2 \alpha)} \quad \text { (dependence error) } \\
+6 A\left(2^{n}\right)^{-\frac{\alpha(1+\alpha)}{1+2 \alpha}+(\rho+\varepsilon)(2+2 \alpha)} \\
\quad+\text { variance homogenization error } \\
+ \text { gaps error + oscillation error). }
\end{gathered}
$$

Taking into account that $\alpha<\delta$ and $2^{n} \leq N<2^{n+1}$ we obtain

$$
\mathbb{P}\left(N^{-\frac{1}{2}} \sup _{1 \leq l \leq N}\left|\sum_{i=1}^{l}\left(\tilde{X}_{i}-W_{i}\right)\right| \geq 6 N^{-\rho}\right) \leq A^{\prime} N^{-\frac{\alpha(1+\alpha)}{1+2 \alpha}+(\rho+\varepsilon)(2+2 \alpha)}
$$

where $A^{\prime}=c_{\varepsilon, \lambda_{1}, \lambda_{2}, \alpha, \delta}\left(1+\lambda_{0}+\mu_{\delta}+\sqrt{\tau}\right)^{2+2 \delta}$ and $\rho$ satisfies $0<\rho<\frac{1-\beta}{2}=\frac{\alpha}{2(1+2 \alpha)}$.

Note that the function $g(\alpha)=\frac{\alpha(1+\alpha)}{1+2 \alpha}-\rho(2+2 \alpha)$ is strictly increasing on $\mathbb{R}^{+}$when $\rho<\frac{1}{4}$. Therefore we can get rid of the constant $\varepsilon$ in the bound by choosing $\alpha^{\prime}<\alpha$. If we let $\Delta=g(\alpha)-g\left(\alpha^{\prime}\right)>0$ and choose $\varepsilon$ sufficiently small, we obtain $\left(2^{n}\right)^{-g(\alpha)+\varepsilon(2+2 \alpha)}=$ $\left(2^{n}\right)^{-g\left(\alpha^{\prime}\right)-\Delta+\varepsilon(2+2 \alpha)} \leq\left(2^{n}\right)^{-g\left(\alpha^{\prime}\right)}$. Since $\alpha$ and $\alpha^{\prime}$ are arbitrary satisfying $\alpha^{\prime}<\alpha<\delta$, the assertion of Theorem 1 follows with $\alpha^{\prime}$ replacing $\alpha$.

We have performed a construction of the sequences $\widetilde{X}^{(N)}=(\widetilde{X})_{1 \leq i \leq N}$ and $W^{(N)}=$ $(W)_{1 \leq i \leq N}$ for each fixed $N \geq 1$, where for each $N$ the constructed sequences in general are different. Below we show how to obtain a construction of the entire sequences $(\widetilde{X})_{i \geq 1}$ and $(W)_{i>1}$.

Let $\Omega^{(N)}=\mathbb{R}^{N+1} \times \mathbb{R}^{N+1}$. Without loss of generality, for any $\omega=\left(\omega_{1}, \omega_{2}\right) \in \Omega^{(N)}$, the sequences $\widetilde{X}^{(N)}$ and $W^{(N)}$ can be reconstructed on $\Omega^{(N)}$ so that $\tilde{X}_{i}=\omega_{1, i}, W_{i}=\omega_{2, i}$ and their joint distribution, say $\mathbb{P}^{(N)}$, is preserved. Each measure $\mathbb{P}^{(N)}$ can be extended (arbitrarily) on the space $\mathbb{R}^{\infty} \times \mathbb{R}^{\infty}$. From the bound (6.19) it follows that the sequence of measures $\mathbb{P}^{(N)}$ is tight. Therefore there is a weak limit which satisfies (6.19) and thus provides the desired construction.

\section{Appendix 1}

7.1. Some general bounds for the Prokhorov distance. Let $(E, d)$ be a metric space endowed with the metric $d$ and $\mathcal{E}$ be the Borel $\sigma$-algebra on $E$. For any $B \in \mathcal{E}$ denote by $B^{\varepsilon}$ its $\varepsilon$-extension: $B^{\varepsilon}=\{x \in E: d(x, E) \leq \varepsilon\}$. Let $\pi(\mathbf{P}, \mathbf{Q})$ be the Prokhorov distance 
between two probability measures $\mathbf{P}$ and $\mathbf{Q}$ defined by

$$
\pi(\mathbf{P}, \mathbf{Q})=\inf \left\{\varepsilon: \sup _{B \in \mathcal{E}}\left|\mathbf{P}(B)-\mathbf{Q}\left(B^{\varepsilon}\right)\right| \leq \varepsilon\right\} .
$$

The following assertion is known as Strassen-Dudley theorem and is a consequence of the results in Strassen [40] (see also Dudley [8]). Let $\mathcal{P}_{E}(\mathbf{P}, \mathbf{Q})$ be the set of probability measures on $E \times E$ with given marginals $\mathbf{P}$ and $\mathbf{Q}$. Denote by $\mathcal{D}_{E, d}(\varepsilon)$ the $\varepsilon$-extension of the diagonal in $E \times E$, i.e. $\mathcal{D}_{E, d}(\varepsilon)=\left\{\left(s, s^{\prime}\right) \in E \times E: d\left(s, s^{\prime}\right) \leq \varepsilon\right\}$ and by $\overline{\mathcal{D}}_{E, d}(\varepsilon)$ its complement.

Lemma 3. (Strassen-Dudley) If $(E, d)$ is a complete separable metric space, then

$$
\pi(\mathbf{P}, \mathbf{Q})=\min \left\{\varepsilon: \exists \mathbb{P} \in \mathcal{P}_{E}(\mathbf{P}, \mathbf{Q}) \text { such that } \mathbb{P}\left(\overline{\mathcal{D}}_{E, d}(\varepsilon)\right) \leq \varepsilon\right\} .
$$

Let $\left(E_{1}, d_{1}\right)$ and $\left(E_{2}, d_{2}\right)$ be two complete separable metric spaces endowed with Borel $\sigma$ algebras $\mathcal{E}_{1}$ and $\mathcal{E}_{2}$ respectively. Consider the product space $E=E_{1} \times E_{2}$ endowed with the metric $d(x, y)=\max \left\{d_{1}\left(x_{1}, y_{1}\right), d_{2}\left(x_{2}, y_{2}\right)\right\}$, where $x=\left(x_{1}, x_{2}\right), y=\left(y_{1}, y_{2}\right) \in E$. Denote by $\mathcal{E}$ the Borel $\sigma$-algebra on $E$.

Lemma 4. Consider the r.v.'s $X, Y \in E_{1}$ and $Z \in E_{2}$ such that $Z$ and $(X, Y)$ are independent. Then

$$
\pi\left(\mathcal{L}_{X, Z}, \mathcal{L}_{Y, Z}\right)=\pi\left(\mathcal{L}_{X}, \mathcal{L}_{Y}\right)
$$

Proof. Let $\mathbb{P}_{1} \in \mathcal{P}_{E_{1}}\left(\mathcal{L}_{X}, \mathcal{L}_{Y}\right)$ and $\mathbb{P}_{2} \in \mathcal{P}_{E_{2}}\left(\mathcal{L}_{Z}, \mathcal{L}_{Z}\right)$. If $\mathbb{P}_{2}$ is concentrated on the diagonal of $E_{2} \times E_{2}$, then with $\mathbb{P}=\mathbb{P}_{1} \times \mathbb{P}_{2}$ we have $\mathbb{P}\left(\overline{\mathcal{D}}_{E, d}(\varepsilon)\right)=\mathbb{P}_{1}\left(\overline{\mathcal{D}}_{E_{1}, d_{1}}(\varepsilon)\right)$. This means that

$$
\begin{aligned}
& A=\left\{\varepsilon: \exists \mathbb{P}_{1} \in \mathcal{P}_{E}\left(\mathcal{L}_{X}, \mathcal{L}_{Y}\right) \text { such that } \mathbb{P}_{1}\left(\overline{\mathcal{D}}_{E_{1}, d_{1}}(\varepsilon)\right) \leq \varepsilon\right\} \\
= & \left\{\varepsilon: \exists \mathbb{P} \in \mathcal{P}_{E}\left(\mathcal{L}_{X, Z}, \mathcal{L}_{Y, Z}\right) \text { such that } \mathbb{P}\left(\overline{\mathcal{D}}_{E, d}(\varepsilon)\right) \leq \varepsilon\right\}=B .
\end{aligned}
$$

By Lemma $3, \pi\left(\mathcal{L}_{X}, \mathcal{L}_{Y}\right)=\inf A=\inf B=\pi\left(\mathcal{L}_{X, Z}, \mathcal{L}_{Y, Z}\right)$.

Let $\left(E_{1}, d_{1}\right), \ldots,\left(E_{n}, d_{n}\right)$ be complete separable metric spaces. On the product space $E=$ $E_{1} \times \ldots \times E_{n}$ consider the metric $d(x, y)=\max \left\{d_{1}\left(x_{1}, y_{1}\right), \ldots, d_{n}\left(x_{n}, y_{n}\right)\right\}$, where $x=$ $\left(x_{1}, \ldots, x_{n}\right), y=\left(y_{1}, \ldots, y_{n}\right) \in E$.

Lemma 5. Consider the r.v.'s $X=\left(X_{1}, \ldots, X_{n}\right) \in E$ and $Y=\left(Y_{1}, \ldots, Y_{n}\right) \in E$. If $X$ and $Y$ are independent and $Y_{1}, \ldots, Y_{n}$ are independent, then

$$
\pi\left(\mathcal{L}_{X_{1}, \ldots, X_{n}}, \mathcal{L}_{Y_{1}, \ldots, Y_{n}}\right) \leq \sum_{k=1}^{n} \pi\left(\mathcal{L}_{X_{1}, \ldots, X_{k-1}, X_{k}}, \mathcal{L}_{X_{1}, \ldots, X_{k-1}, Y_{k}}\right) .
$$

Proof. The assertion of the lemma is obtained using the telescope rule and Lemma 4.

Lemma 6. Consider the r.v.'s $X=\left(X_{1}, \ldots, X_{n}\right) \in E$ and $Y=\left(Y_{1}, \ldots, Y_{n}\right) \in E$. If $\left(X_{1}, Y_{1}\right) \ldots,\left(X_{n}, Y_{n}\right)$ are independent, then

$$
\pi\left(\mathcal{L}_{X_{1}, \ldots, X_{n}}, \mathcal{L}_{Y_{1}, \ldots, Y_{n}}\right) \leq \sum_{k=1}^{n} \pi\left(\mathcal{L}_{X_{k}}, \mathcal{L}_{Y_{k}}\right)
$$

Proof. The assertion of the lemma is obtained using Lemma 5 and Lemma 4. 
Lemma 7. Let $\mathbf{P}$ and $\mathbf{Q}$ be two probability measures on $\left(\mathbb{R}^{N}, \mathcal{B}^{N}\right)$. Denote by $\widehat{p}(t)$ and $\widehat{q}(t)$ the characteristic functions pertaining to $\mathbf{P}$ and $\mathbf{Q}$ respectively. Then

$$
\pi(\mathbf{P}, \mathbf{Q}) \leq(T / \pi)^{N / 2}\left(\int_{\mathbb{R}^{N}}|\widehat{p}(t)-\widehat{q}(t)|^{2} d t\right)^{1 / 2}+\mathbf{P}\left(\|x\|_{\infty}>T\right) .
$$

Proof. Assume first that $\mathbf{P}, \mathbf{Q}$ have square integrable densities $p$ and $q$ respectively. Denote $C_{T}=\left\{(x, y) \in \mathbb{R}^{N}:\|x\|_{\infty} \leq T\right\}$ and $B_{T}=\mathbb{R}^{N} \backslash C_{T}$. Assume that $A \in \mathcal{B}^{N}$ and let $\varepsilon>0$. Then

$$
\begin{aligned}
& \left|\mathbf{P}(A)-\mathbf{Q}\left(A^{\varepsilon}\right)\right| \\
= & \left|\mathbf{P}\left(A^{\varepsilon} \cap C_{T}\right)+\mathbf{P}\left(A^{\varepsilon} \cap B_{T}\right)-\mathbf{Q}\left(A^{\varepsilon}\right)\right| \\
\leq & \left|\mathbf{P}\left(A^{\varepsilon} \cap C_{T}\right)-\mathbf{Q}\left(A^{\varepsilon} \cap C_{T}\right)\right|+\mathbf{P}\left(B_{T}\right) \\
= & \left|\int_{A^{\varepsilon} \cap C_{T}}(p(x)-q(x)) d x\right|+\mathbf{P}\left(B_{T}\right) \\
\leq & \int_{\mathbb{R}^{N}}|p(x)-q(x)| 1_{C_{T}}(x) d x+\mathbf{P}\left(\|x\|_{\infty}>T\right) .
\end{aligned}
$$

Using Hölder's inequality, we get

$$
\begin{aligned}
\pi(\mathbf{P}, \mathbf{Q}) & \leq\left|\mathbf{P}(A)-\mathbf{Q}\left(A^{\varepsilon}\right)\right| \\
& \leq\left(\int_{\mathbb{R}^{N}}|p(x)-q(x)|^{2} d x\right)^{1 / 2}\left(\int_{\mathbb{R}^{N}} 1_{C_{T}}(x) d x\right)^{1 / 2}+\mathbf{P}\left(\|x\|_{\infty}>T\right) .
\end{aligned}
$$

Since, by Plancherel's identity

$$
\int_{\mathbb{R}^{N}}|p(x)-q(x)|^{2} d x=(2 \pi)^{-N} \int_{\mathbb{R}^{N}}|\widehat{p}(t)-\widehat{q}(t)|^{2} d t
$$

we obtain (7.1) for $\mathbf{P}$ and $\mathbf{Q}$ having square integrable densities.

If $\mathbf{P}$ and $\mathbf{Q}$ do not have densities, denote by $\mathbf{P}_{v}=\mathbf{P} * \mathbf{G}_{v}$ and $\mathbf{Q}_{v}=\mathbf{Q} * \mathbf{G}_{v}$ the smoothed versions of $\mathbf{P}$ and $\mathbf{Q}$, where $\mathbf{G}_{v}$ is the normal distribution of mean 0 and variance $v^{2}$. Using (7.1) and the obvious inequality $\left|\widehat{p}_{v}(t)-\widehat{q}_{v}(t)\right| \leq|\widehat{p}(t)-\widehat{q}(t)| e^{-v^{2} t^{2} / 2} \leq|\widehat{p}(t)-\widehat{q}(t)|$, we obtain

$$
\pi\left(\mathbf{P}_{v}, \mathbf{Q}_{v}\right) \leq(2 \pi)^{-N / 2}(2 T)^{N / 2}\left(\int_{\mathbb{R}^{N}}|\widehat{p}(t)-\widehat{q}(t)|^{2} d t\right)^{1 / 2}+\mathbf{P}_{v}\left(\|x\|_{\infty}>T\right) .
$$

Since $\pi\left(\mathbf{P}_{v}, \mathbf{P}\right) \rightarrow 0$ and $\pi\left(\mathbf{Q}_{v}, \mathbf{Q}\right) \rightarrow 0$ it follows that $\pi\left(\mathbf{P}_{v}, \mathbf{Q}_{v}\right) \rightarrow \pi(\mathbf{P}, \mathbf{Q})$ as $v \rightarrow 0$. Note also that $\limsup _{v \rightarrow 0} \mathbf{P}_{v}\left(\|x\|_{\infty}>T\right) \leq \mathbf{P}\left(\|x\|_{\infty} \geq T\right)$. The inequality (7.1) follows for arbitrary $\mathbf{P}, \mathbf{Q}$.

7.2. Coupling independent and Gaussian r.v.'s. The following result is proved in Theorem 5 of Sakhanenko [35] (see also [34], [36], [37] for related results). Let $X_{1}, \ldots, X_{n}$ be a sequence of independent r.v.'s satisfying $\mathbb{E} X_{i}=0$ and $\mathbb{E}\left|X_{i}\right|^{p}<\infty$ for some $p \geq 2$ and all $1 \leq i \leq n$. 
Theorem 4. On some probability space $\left(\Omega^{\prime}, \mathcal{F}^{\prime}, \mathbb{P}^{\prime}\right)$ there is a sequence of independent normal r.v.'s $Y_{1}, \ldots, Y_{n}$ satisfying $\mathbb{E}^{\prime} Y_{i}=0$ and $\mathbb{E}^{\prime} Y_{i}^{2}=\mathbb{E} X_{i}^{2}, 1 \leq i \leq n$, and a sequence of independent r.v.'s $X_{1}^{\prime}, \ldots, X_{n}^{\prime}$ satisfying $X_{i}^{\prime} \stackrel{d}{=} X_{i}, 1 \leq i \leq n$, such that

$$
\mathbb{E}^{\prime}\left(\max _{1 \leq k \leq n}\left|\sum_{i=1}^{k} X_{i}^{\prime}-\sum_{i=1}^{k} Y_{i}\right|\right)^{p} \leq c_{p} \sum_{i=1}^{n} \mathbb{E}\left|X_{i}\right|^{p},
$$

where $c_{p}$ is a positive constant depending only on $p$.

In particular, by Chebyshev's inequality, for the same construction and any $a>0$ it holds

$$
\mathbb{P}^{\prime}\left(\max _{1 \leq k \leq n}\left|\sum_{i=1}^{k} X_{i}^{\prime}-\sum_{i=1}^{k} Y_{i}\right|>a\right) \leq \frac{c_{p}}{a^{p}} \sum_{i=1}^{n} \mathbb{E}\left|X_{i}\right|^{p} .
$$

\section{MAXimal ineQualities}

In this section we state two bounds which are used repeatedly in the paper. The first one gives a control for the $L_{p}$-norm of the maxima of the partial sums of a sequence of dependent r.v.'s. This proposition is a consequence of the second one which give a control of the $L_{p}$-norm of the partial sums of a sequence of dependent r.v.'s.

Proposition 6. Assume conditions $\boldsymbol{C 1}$ and $\boldsymbol{C 2}$. Let $\delta^{\prime}\langle\delta$ and $\epsilon>0$. Then, there is a constant $c_{\lambda_{1}, \lambda_{2}, \delta, \delta^{\prime}, \epsilon}$ depending only on its indices such that for any $m, n \geq 1$ it holds

$$
\left\|\sup _{1 \leq k \leq n}\left|\sum_{i=m}^{m+k-1} X_{i}\right|\right\|_{L^{2+2 \delta^{\prime}}} \leq c_{\lambda_{1}, \lambda_{2}, \delta, \delta^{\prime}, \epsilon}\left(1+\lambda_{0}+\mu_{\delta}\right)^{1+\epsilon} n^{\frac{1}{2}} .
$$

Proof. Denote for brevity $S_{m, n}=\sum_{i=m}^{m+n-1} X_{i}$. Let $\delta^{\prime \prime}$ be such that $\delta^{\prime}<\delta^{\prime \prime}<\delta$. By Proposition 7 (which we assume for the moment), for any $m, n \geq 1$ and $\epsilon>0$ we have $\left\|S_{m, n}\right\|_{L^{2+2 \delta^{\prime \prime}}} \leq A n^{\frac{1}{2}}$, where $A=c_{\lambda_{1}, \lambda_{2}, \delta^{\prime \prime}, \epsilon}\left(1+\lambda_{0}+\mu_{\delta}\right)^{1+\epsilon}$ and $c_{\lambda_{1}, \lambda_{2}, \delta^{\prime \prime}, \epsilon}$ is a constant depending only on its indices. Substituting $S_{m, n}^{\prime}=S_{m, n} / A$, we get $\left\|S_{m, n}^{\prime}\right\|_{L^{2+2 \delta^{\prime \prime}}} \leq n^{\frac{1}{2}}$, for any $m, n \geq 1$. By Theorem A in Serfling [39] (see also Billingsley [4] p. 102), it follows that $\left\|\sup _{1 \leq k \leq n} S_{m, n}^{\prime}\right\|_{L^{2+2 \delta^{\prime \prime}}} \leq n^{\frac{1}{2}} \log _{2}(4 n)$, for any $m, n \geq 1$. Since $\delta^{\prime}<\delta^{\prime \prime}$, it follows that

$$
\left\|\sup _{1 \leq k \leq n} S_{m, n}^{\prime}\right\|_{L^{2+2 \delta^{\prime}}} \leq\left\|\sup _{1 \leq k \leq n} S_{m, n}^{\prime}\right\|_{L^{2+2 \delta^{\prime \prime}}} \leq\left(n^{\frac{1}{2}} \log _{2}(4 n)\right)^{\frac{2+2 \delta^{\prime \prime}}{2+2 \delta^{\prime}}} \leq c_{\delta, \delta^{\prime}} n^{\frac{1}{2}},
$$

from which we deduce $\left\|\sup _{1 \leq k \leq n} S_{m, n}\right\|_{L^{2+2 \delta^{\prime}}} \leq A c_{\delta, \delta^{\prime}} n^{\frac{1}{2}}$.

The following assertion is an adaptation of Proposition 4.1 in Gouëzel [15]. We give an independent proof in order to derive an explicit dependence of the constant involved in the bound on some of the constants in conditions $\mathbf{C 1}$ and $\mathbf{C 2}$. Tracking this explicit dependence plays a crucial role in the proof of Theorem 2 to work out the dependence of the bound on the initial state of the Markov chain $X_{0}=x$. 
Proposition 7. Assume conditions $\boldsymbol{C 1}$ and C2. Let $0<\delta^{\prime}<\delta$ and $\epsilon>0$. Then, there is a constant $c_{\lambda_{1}, \lambda_{2}, \delta^{\prime}, \epsilon}$ depending only on its indices such that, for any $m, n \geq 1$,

$$
\left\|\sum_{i=m}^{m+n-1} X_{i}\right\|_{L^{2+2 \delta^{\prime}}} \leq c_{\lambda_{1}, \lambda_{2}, \delta^{\prime}, \epsilon}\left(1+\lambda_{0}+\mu_{\delta}\right)^{1+\epsilon} n^{\frac{1}{2}} .
$$

The proof of this proposition is given below. First we state several auxiliary assertions.

\subsection{Auxiliary assertions.}

Proposition 8. Assume conditions $\boldsymbol{C 1}$ and $\boldsymbol{C 2}$. Then, for any $\epsilon>0$,

$$
\left\|\sum_{i=m}^{m+n-1} X_{i}\right\|_{L^{2}} \leq c_{\lambda_{1}, \lambda_{2}, \epsilon}\left(1+\lambda_{0}+\mu_{\delta}\right)^{1+\epsilon} n^{\frac{1}{2}},
$$

where $c_{\lambda_{1}, \lambda_{2}, \epsilon}$ is a constant depending only on $\lambda_{1}, \lambda_{2}, \epsilon$.

The proof is based on the following two lemmas.

Lemma 8. Let $u_{n}=\max _{m \geq 1}\left\|\sum_{i=m}^{m+n-1} X_{i}\right\|_{L^{2}}^{2}, n \geq 1$. Assume conditions $\boldsymbol{C 2}$ and $\boldsymbol{C 1}$. Then, for any natural numbers $a, b \geq 1$ and any $\alpha \in\left(0, \frac{1}{2}\right), \gamma \in(0, \delta)$, it holds

$$
u_{a+b} \leq A+u_{a}+u_{b}+\left(c \mu_{\delta}\right)^{2}\left(a^{2 \alpha}+b^{2 \alpha}\right)+c \mu_{\delta}\left(a^{\alpha}+b^{\alpha}\right)\left(A+u_{a}+u_{b}\right)^{1 / 2}+c u_{a}^{1 / 2}+c u_{b}^{1 / 2},
$$

where $c>1$ is an absolute constant, $A=c_{\lambda_{1}, \lambda_{2}, \gamma, \alpha}\left(1+\lambda_{0}+\mu_{\delta}\right)^{2+\gamma}$ and the constant $c_{\lambda_{1}, \lambda_{2}, \gamma, \alpha}$ depends only on $\lambda_{1}, \lambda_{2}, \gamma, \alpha$.

Proof. Let $m \in \mathbb{N}$. Assume that $a \leq b$ (the case $a>b$ is treated in the same manner). Denote $Y_{1}=\sum_{i=m}^{m+a-1} X_{i}, Y_{2}=\sum_{i=m+a+\left[b^{\alpha}\right]}^{m+a+b-1} X_{i}$ and $Y_{0}=\sum_{i=m}^{m+a+b-1} X_{i}$, where $\alpha \in\left(0, \frac{1}{2}\right)$. Note that $Y_{0}=Y_{1}+Y_{2}+Y_{g a p}$, where $Y_{g a p}=\sum_{i=m+a}^{m+a+\left[b^{\alpha}\right]-1} X_{i}$. Therefore

$$
\left\|Y_{0}\right\|_{L^{2}}^{2} \leq\left\|Y_{1}+Y_{2}\right\|_{L^{2}}^{2}+\left\|Y_{\text {gap }}\right\|_{L^{2}}^{2}+2\left\|Y_{1}+Y_{2}\right\|_{L^{2}}\left\|Y_{\text {gap }}\right\|_{L^{2}} \text {. }
$$

In the sequel we shall bound each of the terms in the right-hand side of (8.2).

Let $V_{1}$ and $V_{2}$ be two independent identically distributed r.v.'s of mean 0 , independent of $Y_{1}$ and $Y_{2}$ with a common characteristic function supported in the interval $\left[-\varepsilon_{0}, \varepsilon_{0}\right]$, for some $\varepsilon_{0} \in(0,1)$ and such that $\left\|V_{i}\right\|_{L^{2+2 \delta}} \leq c$, where $c$ is an absolute constant. Denote $\widetilde{Y}_{1}=Y_{1}+V_{1}$ and $\widetilde{Y}_{2}=Y_{2}+V_{2}$. Let $Z_{1}$ and $Z_{2}$ be independent copies of $\widetilde{Y}_{1}$ and $\widetilde{Y}_{2}$. Since $\mathbf{E} e^{i t V_{1}}$ is supported in the interval $\left[-\varepsilon_{0}, \varepsilon_{0}\right]$, by Lemma 7 , for any $T>0$,

$$
\begin{aligned}
\pi\left(\left(\widetilde{Y}_{1}, \widetilde{Y}_{2}\right),\left(Z_{1}, Z_{2}\right)\right) \leq & \frac{T}{\pi}\left(\int_{\left[-\varepsilon_{0}, \varepsilon_{0}\right]^{2}}\left|\left(\phi(t, u)-\psi_{1}(t) \psi_{2}(u)\right)\right|^{2} d t d u\right)^{1 / 2} \\
& +\mathbb{P}\left(\max \left\{\left|\widetilde{Y}_{1}\right|,\left|\widetilde{Y}_{2}\right|\right\}>T\right),
\end{aligned}
$$

where $\phi$ is the characteristic function of the vector $\left(Y_{1}, Y_{2}\right)$ and $\psi_{1}$ and $\psi_{2}$ are the characteristic functions of the r.v.'s $Y_{1}$ and $Y_{2}$. Condition $\mathbf{C 1}$ implies that

$$
\begin{aligned}
\left|\left(\phi(t, u)-\psi_{1}(t) \psi_{2}(u)\right)\right| & \leq \lambda_{0}(1+b)^{2 \lambda_{2}} \exp \left(-\lambda_{1}\left[b^{\alpha}\right]\right) \\
& \leq \lambda_{0} c_{\lambda_{1}}(1+b)^{2 \lambda_{2}} \exp \left(-\lambda_{1} b^{\alpha}\right) .
\end{aligned}
$$


Let $T=e^{\lambda_{1} b^{\alpha} / 2}$. Taking into account that

$$
\begin{aligned}
\mathbb{P}\left(\max \left\{\left|\widetilde{Y}_{1}\right|,\left|\widetilde{Y}_{2}\right|\right\}>T\right) & \leq T^{-1} \mathbb{E} \max \left\{\left|\widetilde{Y}_{1}\right|,\left|\widetilde{Y}_{2}\right|\right\} \\
& \leq T^{-1}\left(\left\|Y_{1}+V_{1}\right\|_{L^{2+2 \delta}}+\left\|Y_{2}+V_{2}\right\|_{L^{2+2 \delta}}\right) \\
& \leq e^{-\frac{\lambda_{1}}{2} b^{\alpha}}\left(c+(a+b) \max _{l \geq 0}\left\|X_{l}\right\|_{L^{2+2 \delta}}\right) \\
& \leq c_{\delta} e^{-\frac{\lambda_{1}}{2} b^{\alpha}} b\left(1+\mu_{\delta}\right)
\end{aligned}
$$

we obtain

$$
\begin{aligned}
\pi\left(\left(\widetilde{Y}_{1}, \widetilde{Y}_{2}\right),\left(Z_{1}, Z_{2}\right)\right) & \leq \frac{1}{\pi} \lambda_{0}(1+b)^{2 \lambda_{2}} e^{-\frac{\lambda_{1}}{2} b^{\alpha}}+c_{\delta} b e^{-\frac{\lambda_{1}}{2} b^{\alpha}}\left(1+\mu_{\delta}\right) \\
& \leq \Delta=c_{\delta, \alpha}(1+b)^{2 \lambda_{2}} e^{-\frac{\lambda_{1}}{2} b^{\alpha}}\left(1+\lambda_{0}+\mu_{\delta}\right)
\end{aligned}
$$

where $c_{\delta, \alpha}>0$ is a constant depending on its indices. According to Strassen-Dudley's theorem there is a coupling of $\left(\widetilde{Y}_{1}, \widetilde{Y}_{2}\right)$ and $\left(Z_{1}, Z_{2}\right)$ such that

$$
P\left(\left\|\left(\widetilde{Y}_{1}, \widetilde{Y}_{2}\right)-\left(Z_{1}, Z_{2}\right)\right\|_{\infty} \geq \Delta\right) \leq \Delta .
$$

Let $S=\widetilde{Y}_{1}+\widetilde{Y}_{2}-\left(Z_{1}+Z_{2}\right)$. Taking into account that $\left\|V_{i}\right\|_{L^{2+2 \delta}} \leq c$, we have

$$
\begin{aligned}
\|S\|_{L^{2+2 \delta}} & =\left\|\widetilde{Y}_{1}+\widetilde{Y}_{2}-\left(Z_{1}+Z_{2}\right)\right\|_{L^{2+2 \delta}} \\
& \leq 2\left\|\widetilde{Y}_{1}+\tilde{Y}_{2}\right\|_{L^{2+2 \delta}} \\
& \leq c(a+b)\left(1+\max _{l \geq 0}\left\|X_{l}\right\|_{L^{2+2 \delta}}\right) \\
& \leq c b\left(1+\mu_{\delta}\right) .
\end{aligned}
$$

Then, for any $\gamma \in(0, \delta)$,

$$
\begin{aligned}
\|S\|_{L^{2}}^{2} \leq & 4 \Delta^{2}+\mathbb{E}|S|^{2} 1(|S| \geq 2 \Delta) \\
\leq & 4 \Delta^{2}+\|S\|_{L^{2+2 \gamma}}^{2} \mathbb{P}(|S| \geq 2 \Delta)^{\frac{\gamma}{1+\gamma}} \\
\leq & 4 \Delta^{2}+c b^{2}\left(1+\mu_{\delta}\right)^{2} \Delta^{\frac{\gamma}{1+\gamma}} \\
\leq & 4 c^{2}(1+b)^{4 \lambda_{2}}\left(1+\lambda_{0}+\mu_{\delta}\right)^{2} e^{-\lambda_{1} b^{\alpha}} \\
& +4 b^{2}\left(1+\mu_{\delta}\right)^{2} c_{\delta, \alpha}^{\frac{\gamma}{1+\gamma}}(1+b)^{4 \lambda_{2} \frac{\gamma}{1+\gamma}} e^{-\frac{\lambda_{1}}{2} \frac{\gamma}{1+\gamma} b^{\alpha}}\left(1+\lambda_{0}+\mu_{\delta}\right)^{\frac{\gamma}{1+\gamma}} \\
\leq & A^{\prime}
\end{aligned}
$$

where $A^{\prime}=c_{\lambda_{1}, \lambda_{2}, \gamma, \alpha}^{\prime}\left(1+\lambda_{0}+\mu_{\delta}\right)^{2+\gamma}$ and $c_{\lambda_{1}, \lambda_{2}, \gamma, \alpha}^{\prime}>1$ is a constant depending only on $\lambda_{1}, \lambda_{2}, \gamma, \alpha$. From (8.3) and (8.4), it follows that

$$
\begin{aligned}
\left\|\widetilde{Y}_{1}+\widetilde{Y}_{2}\right\|_{L^{2}}^{2} & \leq\|S\|_{L^{2}}^{2}+\left\|Z_{1}\right\|_{L^{2}}^{2}+\left\|Z_{2}\right\|_{L^{2}}^{2} \\
& \leq A^{\prime}+\left\|\widetilde{Y}_{1}\right\|_{L^{2}}^{2}+\left\|\widetilde{Y}_{2}\right\|_{L^{2}}^{2} .
\end{aligned}
$$


Since $\left\|V_{i}\right\|_{L^{2}} \leq c$, we have

$$
\left\|Y_{1}+Y_{2}\right\|_{L^{2}} \leq\left\|\widetilde{Y}_{1}+\widetilde{Y}_{2}\right\|_{L^{2}}+2 c .
$$

Taking into account (8.5) and (8.6), one gets

$$
\begin{aligned}
& \left\|Y_{1}+Y_{2}\right\|_{L^{2}}^{2} \\
\leq & A^{\prime}+\left\|\widetilde{Y}_{1}\right\|_{L^{2}}^{2}+\left\|\widetilde{Y}_{2}\right\|_{L^{2}}^{2}+4 c\left\|\widetilde{Y}_{1}\right\|_{L^{2}}+4 c\left\|\widetilde{Y}_{2}\right\|_{L^{2}}+4 c^{2}
\end{aligned}
$$

Since $\left\|\widetilde{Y}_{k}\right\|_{L^{2}} \leq\left\|Y_{k}\right\|_{L^{2}}+c$, it holds

$$
\begin{aligned}
\left\|Y_{1}+Y_{2}\right\|_{L^{2}}^{2} \leq & A^{\prime}+\left(\left\|Y_{1}\right\|_{L^{2}}+c\right)^{2}+\left(\left\|Y_{2}\right\|_{L^{2}}+c\right)^{2} \\
& +4 c\left(\left\|Y_{1}\right\|_{L^{2}}+c\right)+4 c\left(\left\|Y_{2}\right\|_{L^{2}}+c\right)+4 c^{2} \\
\leq & A^{\prime}+\left\|Y_{1}\right\|_{L^{2}}^{2}+\left\|Y_{2}\right\|_{L^{2}}^{2}+6 c\left(\left\|Y_{1}\right\|_{L^{2}}+\left\|Y_{2}\right\|_{L^{2}}\right)+14 c^{2}
\end{aligned}
$$

Since the gap is of size $\left[b^{\alpha}\right]$,

$$
\sup _{m \geq 1}\left\|Y_{g a p}\right\|_{L^{2}} \leq\left[b^{\alpha}\right] \max _{i \geq 1}\left\|X_{i}\right\|_{L^{2+2 \delta}} \leq b^{\alpha} \mu_{\delta} .
$$

From (8.2), (8.8) and (8.9) we obtain

$$
\begin{aligned}
\left\|Y_{0}\right\|_{L^{2}}^{2} \leq & A^{\prime}+\left\|Y_{1}\right\|_{L^{2}}^{2}+\left\|Y_{2}\right\|_{L^{2}}^{2}+6 c\left(\left\|Y_{1}\right\|_{L^{2}}+\left\|Y_{2}\right\|_{L^{2}}\right)+14 c^{2} \\
& +b^{2 \alpha} \mu_{\delta}^{2}+2 b^{\alpha} \mu_{\delta}\left\|Y_{1}+Y_{2}\right\|_{L_{2}} .
\end{aligned}
$$

Taking into account that $\left\|Y_{1}+Y_{2}\right\|_{L^{2}} \leq\left\|Y_{1}\right\|_{L^{2}}+\left\|Y_{2}\right\|_{L^{2}}$ one gets

$$
\begin{aligned}
\left\|Y_{0}\right\|_{L^{2}}^{2} \leq & A^{\prime}+\left\|Y_{1}\right\|_{L^{2}}^{2}+\left\|Y_{2}\right\|_{L^{2}}^{2}+b^{2 \alpha} \mu_{\delta}^{2} \\
& +2 b^{\alpha} \mu_{\delta}\left(\left\|Y_{1}\right\|_{L^{2}}+\left\|Y_{2}\right\|_{L^{2}}\right) \\
& +6 c\left(\left\|Y_{1}\right\|_{L^{2}}+\left\|Y_{2}\right\|_{L^{2}}\right)+14 c^{2}
\end{aligned}
$$

Recal that $u_{a}=\sup _{m \geq 1}\left\|Y_{1}\right\|_{L^{2}}^{2}, u_{b}=\sup _{m \geq 1}\left\|Y_{2}\right\|_{L^{2}}^{2}$ and $u_{a+b}=\sup _{m \geq 1}\left\|Y_{0}\right\|_{L^{2}}^{2}$. Using (8.9) we have $\left\|Y_{2}\right\|_{L^{2}} \leq u_{b}^{1 / 2}+\left\|Y_{g a p}\right\|_{L^{2}} \leq u_{b}^{1 / 2}+b^{\alpha} \mu_{\delta}$. From this and (8.10) we deduce that

$$
\begin{aligned}
u_{a+b} \leq & A^{\prime}+u_{a}+u_{b}+b^{2 \alpha} \mu_{\delta}^{2}+2 b^{\alpha} \mu_{\delta} u_{b}^{1 / 2} \\
& +2 b^{\alpha} \mu_{\delta}\left(u_{a}^{1 / 2}+u_{b}^{1 / 2}+b^{\alpha} \mu_{\delta}\right) \\
& +6 c\left(u_{a}^{1 / 2}+u_{b}^{1 / 2}+b^{\alpha} \mu_{\delta}\right)+14 c^{2} .
\end{aligned}
$$

Rearranging the terms and taking into account that $A^{\prime}>1$, we obtain

$$
\begin{aligned}
u_{a+b} \leq & A^{\prime}+14 c^{2}+u_{a}+u_{b}+3 b^{2 \alpha} \mu_{\delta}^{2} \\
& +b^{\alpha} \mu_{\delta}\left(6 c+u_{a}^{1 / 2}+u_{b}^{1 / 2}\right)+6 c u_{a}^{1 / 2}+6 c u_{b}^{1 / 2},
\end{aligned}
$$

which proves the lemma. 
Lemma 9. Assume that the sequence $\left(u_{n}\right)_{n \geq 1}$ is such that $u_{n}>0$ and

$$
u_{a+b} \leq\left(u_{a}+u_{b}+A\right)+\left(a^{2 \alpha}+b^{2 \alpha}\right) B^{2}+\left(a^{\alpha}+b^{\alpha}\right) B\left(u_{a}+u_{b}+A\right)^{1 / 2}+c u_{a}^{1 / 2}+c u_{b}^{1 / 2},
$$

for all $a, b \geq 1$ and some $A, B>0, \alpha \in\left(0, \frac{1}{2}\right)$. Then

$$
u_{n} \leq c_{\alpha}\left(1+u_{1}+A+B^{2}\right) n,
$$

where $c_{\alpha}$ is a constant depending only on $\alpha$.

Proof. Note that $x y \leq \frac{1}{2}\left(\varepsilon x^{2}+\varepsilon^{-1} y^{2}\right)$, for any $x, y, \varepsilon>0$. Using the assumption of the lemma, we have

$$
\begin{aligned}
u_{a+b} \leq & \left(u_{a}+u_{b}+A\right)+\left(1+\varepsilon^{-1}\right)\left(a^{2 \alpha}+b^{2 \alpha}\right) B^{2} \\
& +\frac{\varepsilon}{2}\left(u_{a}+u_{b}+A\right)+\varepsilon^{-1} c^{2}+\frac{\varepsilon}{2} u_{\alpha}+\frac{\varepsilon}{2} u_{b} \\
\leq & (1+\varepsilon)\left(u_{a}+u_{b}+A\right)+\varepsilon^{-1} c^{2}+\left(1+\varepsilon^{-1}\right)\left(a^{2 \alpha}+b^{2 \alpha}\right) B^{2} .
\end{aligned}
$$

Denote $v_{k}=\max _{1 \leq n \leq 2^{k}} u_{n}, k \geq 0$. From the previous equation it follows that

$$
v_{k+1} \leq(1+\varepsilon)\left(2 v_{k}+A\right)+\varepsilon^{-1} c^{2}+\left(1+\varepsilon^{-1}\right) 2^{2 \alpha k+1} B^{2} .
$$

Dividing by $(2+2 \varepsilon)^{k+1}$ we get

$$
\begin{aligned}
\frac{v_{k+1}}{(2+2 \varepsilon)^{k+1}} & \leq \frac{2 v_{k}+A}{2(2+2 \varepsilon)^{k}}+\left(1+\varepsilon^{-1}\right) \frac{2^{2 \alpha k+1}}{(2+2 \varepsilon)^{k+1}}\left(B^{2}+c^{2}\right) \\
& \leq \frac{v_{k}}{(2+2 \varepsilon)^{k}}+\frac{A}{2(2+2 \varepsilon)^{k}}+\left(1+\varepsilon^{-1}\right) \frac{2^{2 \alpha k+1}}{(2+2 \varepsilon)^{k+1}}\left(B^{2}+c^{2}\right) .
\end{aligned}
$$

Taking into account that $\alpha<\frac{1}{2}$, by induction, we obtain

$$
\begin{aligned}
\frac{v_{k}}{(2+2 \varepsilon)^{k}} & \leq v_{0}+\frac{A}{2} \sum_{i=0}^{\infty} \frac{1}{(2+2 \varepsilon)^{i}}+2\left(1+\varepsilon^{-1}\right)\left(B^{2}+c^{2}\right) \sum_{i=1}^{\infty} \frac{2^{2 \alpha i}}{(2+2 \varepsilon)^{i+1}} \\
& \leq v_{0}+\frac{A}{2} \frac{2+2 \varepsilon}{1+2 \varepsilon}+2\left(1+\varepsilon^{-1}\right)\left(B^{2}+c^{2}\right) \frac{1+\varepsilon}{\varepsilon} \\
& \leq v_{0}+c_{\varepsilon}^{\prime}\left(A+B^{2}+c^{2}\right),
\end{aligned}
$$

where $c_{\varepsilon}^{\prime}$ depends only on $\varepsilon$. This implies that

$$
v_{k}=\max _{1 \leq n \leq 2^{k}} u_{n} \leq C_{0}(2+2 \varepsilon)^{k},
$$

where $C_{0}=\left(v_{0}+c_{\varepsilon}^{\prime}\left(A+B^{2}+c^{2}\right)\right)$. Once again using the assumption of the lemma it follows that

$$
\begin{aligned}
v_{k+1} & \leq\left(2 v_{k}+A\right)+2^{2 \alpha k+1} B^{2}+2^{\alpha k+1} B\left(2 C_{0}(2+2 \varepsilon)^{k}+A\right)^{1 / 2}+2 c C_{0}^{1 / 2}(2+2 \varepsilon)^{k / 2} \\
& \leq\left(2 v_{k}+A\right)+2^{2 \alpha k+1} B^{2}+2^{\alpha k+1} B\left(2 C_{0}^{1 / 2}(2+2 \varepsilon)^{k / 2}+A^{1 / 2}\right)+2 c C_{0}^{1 / 2}(2+2 \varepsilon)^{k / 2} .
\end{aligned}
$$


Dividing by $2^{k+1}$ and choosing $\varepsilon=\varepsilon(\alpha)$ so small that $2+2 \varepsilon \leq 2^{1+\left(\frac{1}{2}-\alpha\right)}$, one gets

$$
\begin{aligned}
\frac{v_{k+1}}{2^{k+1}} \leq & \frac{v_{k}}{2^{k}}+\frac{A}{2^{k+1}}+2^{(2 \alpha-1) k+1} B^{2}+2^{(\alpha-1) k+1} B\left(2 C_{0}^{1 / 2} 2^{\frac{k}{2}+\frac{k}{2}\left(\frac{1}{2}-\alpha\right)}+A^{1 / 2}\right) \\
& +2^{-k} c C_{0}^{1 / 2} 2^{\frac{k}{2}+\frac{k}{2}\left(\frac{1}{2}-\alpha\right)} \\
\leq & \frac{v_{k}}{2^{k}}+\frac{A}{2^{k+1}}+2^{(2 \alpha-1) k+1} B^{2}+4 B C_{0}^{1 / 2} 2^{\left(\alpha-\frac{1}{2}\right) \frac{k}{2}}+2^{(\alpha-1) k+1} B A^{1 / 2} \\
& +c C_{0}^{1 / 2} 2^{-\frac{k}{2}\left(\frac{1}{2}+\alpha\right)} .
\end{aligned}
$$

Using induction, this implies

$$
\frac{v_{k}}{2^{k}} \leq c_{\alpha}\left(A+C_{0}+B^{2}\right)
$$

where $c_{\alpha}$ depends only on $\alpha$ (since $\varepsilon$ depends on $\alpha$ itself). From this we get $u_{2^{k}} \leq D 2^{k}$, for any $k \geq 1$, where $D=c_{\alpha}^{\prime}\left(1+v_{0}+A+B^{2}\right)$. Therefore, for any $2^{k-1} \leq n \leq 2^{k}$ it holds $u_{n} \leq D 2^{k} \leq 2 D 2^{k-1} \leq 2 D n$. Lemma is proved.

Let $\alpha=\frac{1}{4}$. In the notations of Lemma $8 u_{1} \leq \max _{m \geq 1}\left\|X_{m}\right\|_{L^{2+2 \delta}}^{2} \leq \mu_{\delta}^{2}$. From Lemmas 8 and 9 with $B=c \mu_{\delta}$ it follows, for any $\epsilon \in(0, \delta)$,

$$
\begin{aligned}
\max _{m \geq 1}\left\|\sum_{i=m}^{m+n-1} X_{i}\right\|_{L^{2}}^{2} & =u_{n} \leq c_{\alpha}\left(u_{1}+A+c^{2} \mu_{\delta}^{2}\right) n \\
& \leq c\left(c_{\lambda_{1}, \lambda_{2}, \epsilon}\left(1+\lambda_{0}+\mu_{\delta}\right)^{2+\frac{\epsilon}{1+\epsilon}}+2 \mu_{\delta}^{2}\right) n \\
& \leq c_{\lambda_{1}, \lambda_{2}, \epsilon}^{\prime}\left(1+\lambda_{0}+\mu_{\delta}\right)^{2+\epsilon} n,
\end{aligned}
$$

for some constant $c_{\lambda_{1}, \lambda_{2}, \epsilon}^{\prime}$ depending only on $\lambda_{1}, \lambda_{2}, \epsilon$, which proves Proposition 8 .

8.2. Proof of Proposition 7. Let $m, n \in \mathbb{N}$ and $a=\left[n^{1-\alpha}\right]$ and $b=\left[n^{\alpha+\rho}\right]$, where $\alpha>0$, and $\rho>0$ are such that $2 \alpha+\rho<1$. Note that $a>b$ and $b a \leq n^{1-\rho}$. Consider the intervals $I_{k}=[m+(k-1) a, m+k a-b), J_{k}=[m+k a-b, m+k a)$, for $k=1, \ldots,\left[n^{\alpha}\right]$ and $I_{\text {fin }}=[m+b a, m+n)$, such that $[m, m+n)=\cup_{k=1}^{\left[n^{\alpha}\right]}\left(I_{k} \cup J_{k}\right) \cup I_{\text {fin }}$. Here $a-b>0$ and $b>0$ are interpreted as the length of an island $I_{k}$ and the length of a gap $J_{k}$ respectively.

Denote $Y_{k}=\sum_{i \in I_{k}} X_{i}, k=1, \ldots,\left[n^{\alpha}\right]$. Let $V_{1}, \ldots, V_{\left[n^{\alpha}\right]}$ be independent identically distributed r.v.'s of mean 0 , independent of $Y_{1}, \ldots, Y_{\left[n^{\alpha}\right]}$ with a common characteristic function supported in the interval $\left[-\varepsilon_{0}, \varepsilon_{0}\right]$, for some $\varepsilon_{0}>0$ and such that $\left\|V_{k}\right\|_{L^{2+2 \delta}} \leq c$, $k=1, \ldots,\left[n^{\alpha}\right]$, where $c$ is an absolute constant. Denote $\widetilde{Y}_{k}=Y_{k}+V_{k}$. Let $Z_{1}, \ldots, Z_{\left[n^{\alpha}\right]}$ be independent copies of $\widetilde{Y}_{1}, \ldots, \widetilde{Y}_{\left[n^{\alpha}\right]}$. By Lemma 5

$$
\pi\left(\left(\widetilde{Y}_{1}, \ldots, \widetilde{Y}_{\left[n^{\alpha}\right]}\right),\left(Z_{1}, \ldots, Z_{\left[n^{\alpha}\right]}\right)\right) \leq \sum_{k=1}^{\left[n^{\alpha}\right]} \pi\left(\left(\widetilde{Y}_{1}, \ldots, \widetilde{Y}_{k-1}, \widetilde{Y}_{k}\right),\left(\widetilde{Y}_{1}, \ldots, \widetilde{Y}_{k-1},, Z_{k}\right)\right)
$$


Since $\mathbf{E} e^{i t V_{k}}$ is supported in the interval $\left[-\varepsilon_{0}, \varepsilon_{0}\right]$, by Lemma 7 , for any $T>0$ and $k \leq\left[n^{\alpha}\right]$,

$$
\begin{aligned}
& \pi\left(\left(\widetilde{Y}_{1}, \ldots, \widetilde{Y}_{k-1}, \widetilde{Y}_{k}\right),\left(\widetilde{Y}_{1}, \ldots, \widetilde{Y}_{k-1},, Z_{k}\right)\right) \\
\leq & \frac{T}{\pi}\left(\int_{\left[-\varepsilon_{0}, \varepsilon_{0}\right]^{k}}\left|\phi(t, u)-\psi_{1}(t) \psi_{2}(u)\right|^{2} d t d u\right)^{1 / 2} \\
& +\mathbb{P}\left(\left\|\left(\tilde{Y}_{1}, \ldots, \widetilde{Y}_{k-1}, \widetilde{Y}_{k}\right)\right\|_{\infty}>T\right),
\end{aligned}
$$

where $\phi$ is the characteristic function of $\left(\widetilde{Y}_{1}, \ldots, \widetilde{Y}_{k-1}, \widetilde{Y}_{k}\right)$ and $\psi_{1}$ and $\psi_{2}$ are the characteristic functions of the r.v.'s $\left(\widetilde{Y}_{1}, \ldots, \widetilde{Y}_{k-1}\right)$ and $\widetilde{Y}_{k}$. Condition $\mathbf{C 1}$ implies that

$$
\left|\phi(t, u)-\psi_{1}(t) \psi_{2}(u)\right| \leq \lambda_{0}(1+a)^{k} \exp \left(-\lambda_{1} b\right) \leq c_{\lambda_{1}} \lambda_{0}\left(1+n^{1-\alpha}\right)^{n^{\alpha}} \exp \left(-\lambda_{1} n^{\alpha+\rho}\right) .
$$

Let $T=e^{\frac{\lambda_{1}}{2} n^{\alpha+\rho}}$. By Chebychev's inequality, taking into account that for $k \leq\left[n^{\alpha}\right]$, we have

$$
\begin{aligned}
\mathbb{P}\left(\left\|\left(\widetilde{Y}_{1}, \ldots, \widetilde{Y}_{k-1}, \widetilde{Y}_{k}\right)\right\|_{\infty}>T\right) & \leq T^{-1} \sum_{i=1}^{k}\left\|Y_{i}+V_{i}\right\|_{L^{1}} \\
& \leq T^{-1} \sum_{i=1}^{k}\left(\left\|Y_{i}\right\|_{L^{2+2 \delta}}+c\right) \\
& \leq e^{-\frac{\lambda_{1}}{2} n^{\alpha+\rho}}\left[n^{\alpha}\right]\left(\left[n^{1-\alpha}\right] \mu_{\delta}+c\right) \\
& \leq c n e^{-\frac{\lambda_{1}}{2} n^{\alpha+\rho}}\left(1+\mu_{\delta}\right) .
\end{aligned}
$$

From (8.12), (8.13) and (8.14) we obtain

$$
\begin{aligned}
& \pi\left(\left(\widetilde{Y}_{1}, \ldots, \widetilde{Y}_{k-1}, \widetilde{Y}_{k}\right),\left(\widetilde{Y}_{1}, \ldots, \widetilde{Y}_{k-1},, Z_{k}\right)\right) \\
\leq & \frac{T}{\pi} \lambda_{0} \varepsilon_{0}^{n}\left(1+n^{1-\alpha}\right)^{n^{\alpha}} e^{-\lambda_{1} n^{\alpha+\rho}}+c_{\alpha} n e^{-\frac{\lambda_{1}}{2} n^{\alpha+\rho}}\left(1+\mu_{\delta}\right) \\
\leq & c \varepsilon_{0}^{n} n\left(1+n^{1-\alpha}\right)^{n^{\alpha}} e^{-\frac{\lambda_{1}}{2} n^{\alpha+\rho}}\left(1+\lambda_{0}+\mu_{\delta}\right),
\end{aligned}
$$

where $c$ is an absolute constant. Implementing this bound in (8.11) we get

$$
\begin{aligned}
\pi\left(\left(\widetilde{Y}_{1}, \ldots, \widetilde{Y}_{\left[n^{\alpha}\right]}\right),\left(Z_{1}, \ldots, Z_{\left[n^{\alpha}\right]}\right)\right) & \leq c \varepsilon_{0}^{n} n^{1+\alpha}\left(1+n^{1-\alpha}\right)^{n^{\alpha}} e^{-\frac{\lambda_{1}}{2} n^{\alpha+\rho}}\left(1+\lambda_{0}+\mu_{\delta}\right) \\
& \leq \Delta=c_{\alpha, \lambda_{1}} e^{-\frac{\lambda_{1}}{4} n^{\alpha+\rho}}\left(1+\lambda_{0}+\mu_{\delta}\right) .
\end{aligned}
$$

According to Strassen-Dudley's theorem there is a coupling of $\left(\widetilde{Y}_{1}, \ldots, \widetilde{Y}_{b}\right)$ and $\left(Z_{1}, \ldots, Z_{b}\right)$ such that

$$
P\left(\left\|\left(\widetilde{Y}_{1}, \ldots, \widetilde{Y}_{\left[n^{\alpha}\right]}\right)-\left(Z_{1}, \ldots, Z_{\left[n^{\alpha}\right]}\right)\right\|_{\infty} \geq \Delta\right) \leq \Delta
$$


Let $S=\widetilde{Y}_{1}+\ldots+\widetilde{Y}_{\left[n^{\alpha}\right]}-\left(Z_{1}+\ldots+Z_{\left[n^{\alpha}\right]}\right)$. Taking into account that $\left\|V_{i}\right\|_{L^{2+2 \delta}} \leq c$, we have

$$
\begin{aligned}
\|S\|_{L^{2+2 \delta}} & =\left\|\widetilde{Y}_{1}+\ldots+\widetilde{Y}_{\left[n^{\alpha}\right]}-\left(Z_{1}+\ldots+Z_{\left[n^{\alpha}\right]}\right)\right\|_{L^{2+2 \delta}} \\
& \leq c n^{\alpha} a\left(1+\max _{l \geq 1}\left\|X_{l}\right\|_{L^{2+2 \delta}}\right) \leq c^{\prime} n\left(1+\mu_{\delta}\right) .
\end{aligned}
$$

Let $\eta \in\left(0, \delta-\delta^{\prime}\right), p=2+2 \delta^{\prime}, p^{\prime}=p+2 \eta \leq 2+2 \delta$ and $\gamma=\gamma(\eta)=\frac{2 \eta}{p(p+2 \eta)}$. By Hölder's inequality,

$$
\left\|S^{2+2 \delta^{\prime}} 1\left(|S| \geq n^{\alpha} \Delta\right)\right\|_{L^{2+2 \delta^{\prime}}} \leq\|S\|_{L^{p^{\prime}}}\left(\mathbb{P}\left(|S| \geq n^{\alpha} \Delta\right)\right)^{\gamma} \leq\|S\|_{L^{2+2 \delta}}\left(\mathbb{P}\left(|S| \geq n^{\alpha} \Delta\right)\right)^{\gamma} .
$$

Using the bound $|S| \leq n^{\alpha}\left\|\left(\widetilde{Y}_{1}, \ldots, \widetilde{Y}_{\left[n^{\alpha}\right]}\right)-\left(Z_{1}, \ldots, Z_{\left[n^{\alpha}\right]}\right)\right\|_{\infty}$ and we have

$$
\begin{aligned}
\|S\|_{L^{2+2 \delta^{\prime}}} & \leq n^{\alpha} \Delta+\left\|S^{2+2 \delta^{\prime}} 1\left(|S| \geq n^{\alpha} \Delta\right)\right\|_{L^{2+2 \delta^{\prime}}} \\
& \leq n^{\alpha} \Delta+\|S\|_{L^{2+2 \delta}}\left(\mathbb{P}\left(|S| \geq n^{\alpha} \Delta\right)\right)^{\gamma} \\
& \leq n^{\alpha} \Delta+\|S\|_{L^{2+2 \delta}}\left(\mathbb{P}\left(\left\|\left(\widetilde{Y}_{1}, \ldots, \widetilde{Y}_{\left[n^{\alpha}\right]}\right)-\left(Z_{1}, \ldots, Z_{\left[n^{\alpha}\right]}\right)\right\|_{\infty} \geq \Delta\right)\right)^{\gamma} \\
& \leq n^{\alpha} \Delta+c n\left(1+\mu_{\delta}\right) \Delta^{\gamma} .
\end{aligned}
$$

Taking into account the definition of $\Delta$,

$$
\begin{aligned}
\|S\|_{L^{2+2 \delta^{\prime}} \leq} \leq & n^{\alpha} c_{\alpha, \lambda_{1}} e^{-\frac{\lambda_{1}}{4} n^{\alpha+\rho}}\left(1+\lambda_{0}+\mu_{\delta}\right) \\
& +c n\left(1+\mu_{\delta}\right)\left(c_{\alpha, \lambda_{1}} e^{-\frac{\lambda_{1}}{4} n^{\alpha+\rho}}\left(1+\lambda_{0}+\mu_{\delta}\right)\right)^{\gamma} \\
\leq & A^{\prime}=c_{\lambda_{1}, \lambda_{2}, \gamma, \alpha, \rho}^{\prime}\left(1+\lambda_{0}+\mu_{\delta}\right)^{1+\gamma}
\end{aligned}
$$

where $c_{\lambda_{1}, \lambda_{2}, \gamma, \alpha, \rho}^{\prime}$ is a constant depending only on $\lambda_{1}, \lambda_{2}, \gamma, \alpha, \rho$. From (8.15) and (8.16), it follows that

$$
\begin{aligned}
\left\|\widetilde{Y}_{1}+\ldots+\widetilde{Y}_{\left[n^{\alpha}\right]}\right\|_{L^{2+2 \delta^{\prime}}} & \leq\|S\|_{L^{2+2 \delta^{\prime}}}+\left\|Z_{1}+\ldots+Z_{\left[n^{\alpha}\right]}\right\|_{L^{2+2 \delta^{\prime}}} \\
& \leq A^{\prime}+\left\|Z_{1}+\ldots+Z_{\left[n^{\alpha}\right]}\right\|_{L^{2+2 \delta^{\prime}}} .
\end{aligned}
$$

Since the r.v.'s $Z_{1}, \ldots, Z_{\left[n^{\alpha}\right]}$ are independent, by Rosenthal's inequality [33], there exists some constant $c_{\delta^{\prime}}$, depending only on $\delta^{\prime}$, such that

$$
\left\|Z_{1}+\ldots+Z_{\left[n^{\alpha}\right]}\right\|_{L^{2+2 \delta^{\prime}}} \leq c_{\delta^{\prime}}\left(\sum_{i=1}^{\left[n^{\alpha}\right]} \mathbb{E} Z_{i}^{2}\right)^{\frac{1}{2}}+c_{\delta^{\prime}}\left(\sum_{i=1}^{\left[n^{\alpha}\right]} \mathbb{E}\left|Z_{i}\right|^{2+2 \delta^{\prime}}\right)^{\frac{1}{2+2 \delta^{\prime}}}
$$

Taking into account that $Y_{i}=\sum_{j \in I_{i}} X_{j}$ and that $\left|I_{i}\right| \leq a-b \leq n^{1-\alpha}$, by Proposition 8 we have

$$
\mathbf{E} Z_{i}^{2}=\left\|\widetilde{Y}_{i}\right\|_{L^{2}}^{2} \leq\left(c+\left\|Y_{i}\right\|_{L^{2}}\right)^{2} \leq c_{\lambda_{1}, \lambda_{2}, \gamma}^{\prime}\left(1+\lambda_{0}+\mu_{\delta}\right)^{2+\gamma} n^{1-\alpha}
$$


Note also that $\left\|Z_{i}\right\|_{L^{2+2 \delta^{\prime}}} \leq v_{a-b}+c$, where $v_{n}=\sup _{m \geq 1}\left\|\sum_{i=m}^{m+n-1} X_{i}\right\|_{L^{2+2 \delta^{\prime}}}$ and $c$ is an absolute constant. Therefore, from (8.18) and (8.19), it follows that

$$
\begin{aligned}
& \left\|Z_{1}+\ldots+Z_{b}\right\|_{L^{2+2 \delta^{\prime}}} \\
\leq & A^{\prime}+c_{\lambda_{1}, \lambda_{2}, \gamma, \alpha, \rho, \delta^{\prime}}\left(1+\lambda_{0}+\mu_{\delta}\right)^{1+\frac{\gamma}{2}} n^{\frac{1}{2}}+c_{\delta^{\prime}}\left(\sum_{i=1}^{\left[n^{\alpha}\right]}\left(v_{a-b}+c\right)^{2+2 \delta^{\prime}}\right)^{\frac{1}{2+2 \delta^{\prime}}} \\
\leq & c_{\lambda_{1}, \lambda_{2}, \gamma, \alpha, \rho, \delta^{\prime}}\left(1+\lambda_{0}+\mu_{\delta}\right)^{1+\gamma} n^{\frac{1}{2}}+c_{\delta^{\prime}} v_{a-b} n^{\frac{\alpha}{2+2 \delta^{\prime}}}
\end{aligned}
$$

Using (8.17), we get

$$
\begin{aligned}
\left\|\widetilde{Y}_{1}+\ldots+\widetilde{Y}_{\left[n^{\alpha}\right]}\right\|_{L^{2+2 \delta^{\prime}}} & \leq A^{\prime}+\left\|Z_{1}+\ldots+Z_{\left[n^{\alpha}\right]}\right\|_{L^{2+2 \delta^{\prime}}} \\
& \leq c_{\lambda_{1}, \lambda_{2}, \gamma, \alpha, \rho, \delta^{\prime}}^{\prime}\left(1+\lambda_{0}+\mu_{\delta}\right)^{1+\gamma} n^{\frac{1}{2}}+c_{\delta^{\prime}} v_{a-b} n^{\frac{\alpha}{2+2 \delta^{\prime}}} .
\end{aligned}
$$

Since $\widetilde{Y}_{k}=Y_{k}+V_{k}$ and $\left\|V_{k}\right\|_{L^{2+2 \delta}} \leq c$, it holds

$$
\begin{aligned}
\left\|Y_{1}+\ldots+Y_{\left[n^{\alpha}\right]}\right\|_{L^{2+2 \delta^{\prime}}} & \leq c\left[n^{\alpha}\right]+\left\|\widetilde{Y}_{1}+\ldots+\widetilde{Y}_{\left[n^{\alpha}\right]}\right\|_{L^{2+2 \delta^{\prime}}} \\
& \leq c n^{\alpha}+c_{\lambda_{1}, \lambda_{2}, \gamma, \alpha, \rho, \delta^{\prime}}\left(1+\lambda_{0}+\mu_{\delta}\right)^{1+\gamma} n^{\frac{1}{2}}+c_{\delta^{\prime}} v_{a-b} n^{\frac{\alpha}{2+2 \delta^{\prime}}} \\
& \leq c_{\lambda_{1}, \lambda_{2}, \gamma, \alpha, \rho, \delta^{\prime}}\left(1+\lambda_{0}+\mu_{\delta}\right)^{1+\gamma} n^{\frac{1}{2}}+c_{\delta^{\prime}} v_{a-b} n^{\frac{\alpha}{2+2 \delta^{\prime}}}
\end{aligned}
$$

where for the last line we use the fact that $\alpha<\frac{1-\rho}{2}<\frac{1}{2}$. Filling up the gaps in the final interval $I_{\text {fin }}$, we get

$$
\begin{aligned}
\left\|\sum_{i=1}^{m+n-1} X_{i}\right\|_{L^{2+2 \delta^{\prime}}} \leq & \left\|Y_{1}+\ldots+Y_{\left[n^{\alpha}\right]}\right\|_{L^{2+2 \delta^{\prime}}}+\sum_{k=1}^{\left[n^{\alpha}\right]} \sum_{i \in J_{k}}\left\|X_{i}\right\|_{L^{2+2 \delta^{\prime}}}+\left\|\sum_{i=m+a\left[n^{\alpha}\right]} X_{i}\right\|_{L^{2+2 \delta^{\prime}}} \\
\leq & c_{\lambda_{1}, \lambda_{2}, \gamma, \alpha, \rho, \delta^{\prime}}\left(1+\lambda_{0}+\mu_{\delta}\right)^{1+\gamma} n^{\frac{1}{2}}+c_{\delta^{\prime}} v_{a-b} n^{\frac{\alpha}{2+2 \delta^{\prime}}} \\
& +n^{2 \alpha+\rho} \mu_{\delta}+v_{n-\left[n^{1-\alpha}\right]\left[n^{\alpha}\right]} .
\end{aligned}
$$

From this, we deduce the inequality

(8.20) $v_{n} \leq c_{\lambda_{1}, \lambda_{2}, \gamma, \alpha, \rho, \delta^{\prime}}\left(1+\lambda_{0}+\mu_{\delta}\right)^{1+\gamma} n^{\frac{1}{2}}+n^{2 \alpha+\rho} \mu_{\delta}+c_{\delta^{\prime}} v_{\left[n^{1-\alpha}\right]-\left[n^{\alpha+\rho}\right]} n^{\frac{\alpha}{2+2 \delta^{\prime}}}+v_{n-\left[n^{1-\alpha}\right]\left[n^{\alpha}\right]}$.

Denote $\bar{v}_{n}=\frac{v_{n}}{\left(1+\lambda_{0}+\mu_{\delta}\right)^{1+\gamma}}$. Then from (8.20), it follows that

$$
\bar{v}_{n} \leq c_{\lambda_{1}, \lambda_{2}, \gamma, \alpha, \rho, \delta^{\prime}} n^{\frac{1}{2}}+n^{2 \alpha+\rho}+c_{\delta^{\prime}} \bar{v}_{\left[n^{1-\alpha}\right]-\left[n^{\alpha+\rho}\right]} n^{\frac{\alpha}{2+2 \delta^{\prime}}}+\bar{v}_{n-\left[n^{1-\alpha}\right]\left[n^{\alpha}\right]} .
$$

Fixing $\alpha=\frac{1}{6}$ and $\rho=\frac{1}{6}$, we get

$$
\bar{v}_{n} \leq c_{\lambda_{1}, \lambda_{2}, \gamma, \delta^{\prime}} n^{\frac{1}{2}}+c_{\delta^{\prime}} \bar{v}_{\left[n^{\frac{5}{6}}\right]-\left[n^{\frac{1}{3}}\right]} n^{\frac{1}{6} \frac{1}{2+2 \delta^{\prime}}}+\bar{v}_{n-\left[n^{\frac{5}{6}}\right]\left[n^{\frac{1}{6}}\right]} .
$$

We start with the inequality $\bar{v}_{n} \leq n^{q_{0}}$, where $q_{0}=1$. Since $n-\left[n^{1-\alpha}\right]\left[n^{\alpha}\right] \leq c n^{1-\alpha}$, we have $v_{n-\left[n^{1-\alpha}\right]\left[n^{\alpha}\right]} \leq c n^{\frac{5}{6} q_{0}}$ and $v_{\left[n^{1-\alpha}\right]-\left[n^{\alpha+\rho}\right]} \leq c n^{\frac{5}{6} q_{0}}$. Implementing this in (8.21) gives,

$$
\bar{v}_{n} \leq c_{\lambda_{1}, \lambda_{2}, \gamma, \delta^{\prime}} n^{\frac{1}{2}}+c_{\delta^{\prime}} n^{\frac{5}{6} q_{0}+\frac{1}{6} \frac{1}{2+2 \delta^{\prime}}} \leq c_{1 r_{1}, \gamma, \delta^{\prime}} n^{\max \left\{\frac{1}{2}, q_{1}\right\}},
$$


where $q_{1}=\frac{5}{6} q_{0}+\frac{1}{6} \frac{1}{2+2 \delta^{\prime}}$. Continuing in the same way, at the iteration $k+1$, we obtain

$$
\bar{v}_{n} \leq c_{k r_{1}, \gamma, \delta^{\prime}} n^{\max \left\{\frac{1}{2}, q_{k+1}\right\}},
$$

where $q_{k+1}=\frac{5}{6} q_{k}+\frac{1}{6} \frac{1}{2+2 \delta^{\prime}}$. Since $\lim _{k \rightarrow \infty} q_{k}=\frac{1}{2+2 \delta^{\prime}}$, there exists a constant $k_{0}<\infty$, such that $q_{k_{0}+1} \leq \frac{1}{2}$. With this $k_{0}$, we get

$$
\bar{v}_{n} \leq c_{k_{0} \lambda_{1}, \lambda_{2}, \gamma, \delta^{\prime}} n^{\frac{1}{2}}
$$

Since $\gamma=\gamma(\eta)=\frac{2 \eta}{p(p+2 \eta)} \leq \frac{2 \eta}{p^{2}}$, we have, for any $m \geq 1$,

$$
\left\|\sum_{i=m}^{m+n} X_{i}\right\|_{L^{2+2 \delta^{\prime}}} \leq c_{\lambda_{1}, \lambda_{2}, \eta, \delta^{\prime}}\left(1+\lambda_{0}+\mu_{\delta}\right)^{1+\gamma} n^{\frac{1}{2}} \leq c_{\lambda_{1}, \lambda_{2}, \delta^{\prime}, \eta}\left(1+\lambda_{0}+\mu_{\delta}\right)^{1+\frac{2 \eta}{p^{2}}} n^{\frac{1}{2}} .
$$

Since $\eta$ is arbitrary we obtain the assertion of Proposition 7.

\section{Appendix 2}

9.1. Proof of Propositions 1, 2 and Theorems 2, 3. First, we establish the following bound for the characteristic functions $\phi_{x, 1}, \phi_{x, 2}$ and $\phi_{x}$ involved in Proposition 1.

Lemma 10. Assume that the Markov chain $\left(X_{n}\right)_{n \geq 0}$ and the function $f$ satisfy the hypotheses M1, M2, $M 3$ and $\mathbf{M}$. Then for any $k_{\text {gap }}, M_{1}, M_{2} \in \mathbb{N}$, any sequence $j_{0}<\ldots<j_{M_{1}+M_{2}}$ and any $t=\left(t_{i}\right)_{i} \in \mathbb{R}^{M_{1}}, s=\left(s_{i}\right)_{i} \in \mathbb{R}^{M_{2}}$ satisfying $\|(t, s)\|_{\infty} \leq \varepsilon_{0}$,

$$
\left|\phi_{x}(t, s)-\phi_{x, 1}(t) \phi_{x, 2}(s)\right| \leq 2 C_{Q} C_{\mathbf{P}}^{M_{1}+M_{2}}\left(\|\nu\|_{\mathcal{B}^{\prime}}+\left\|\delta_{x}\right\|_{\mathcal{B}^{\prime}}\right)\|\mathbf{1}\|_{\mathcal{B}} \kappa^{k_{\text {gap }}} .
$$

Proof. Set for brevity $\phi_{1}=\phi_{x, 1}, \phi_{2}=\phi_{x, 2}$ and $\phi=\phi_{x}$. The characteristic function $\phi$ can be rewritten in the following form:

$$
\phi(t, s)=\left(\mathbf{P}^{j_{0}} \mathbf{P}_{t_{1}}^{\left|J_{1}\right|} \ldots \mathbf{P}_{t_{M_{1}}}^{\left|J_{M_{1}}\right|} \mathbf{P}^{k_{g a p}} \mathbf{P}_{s_{M_{1}+1}}^{\left|J_{M_{1}+1}\right|} \ldots \mathbf{P}_{s_{M_{1}+M_{2}}}^{\left|J_{M_{1}+M_{2}}\right|} \mathbf{1}\right)(x) .
$$

Since $\mathbf{P}=\Pi+Q$ we get $\mathbf{P}^{k}=\Pi+Q^{k}$, and thus

$$
\phi(t, s)=\phi_{\Pi}(t, s)+\phi_{Q}(t, s)
$$

with

$$
\phi_{\Pi}(t, s):=\left(\mathbf{P}^{j_{0}} \mathbf{P}_{t_{1}}^{\left|J_{1}\right|} \ldots \mathbf{P}_{t_{M_{1}}}^{\left|J_{M_{1}}\right|} \Pi^{k_{g a p}} \mathbf{P}_{s_{M_{1}+1}}^{\left|J_{M_{1}+1}\right|} \ldots \mathbf{P}_{s_{M_{1}+M_{2}}}^{\left|J_{M_{1}+M_{2}}\right|} \mathbf{1}\right)(x)
$$

and

$$
\phi_{Q}(t, s):=\left(\mathbf{P}^{j_{0}} \mathbf{P}_{t_{1}}^{\left|J_{1}\right|} \ldots \mathbf{P}_{t_{M_{1}}}^{\left|J_{M_{1}}\right|} Q^{k_{g a p}} \mathbf{P}_{s_{M_{1}+1}}^{\left|J_{M_{1}+1}\right|} \ldots \mathbf{P}_{s_{M_{1}+M_{2}}}^{\left|J_{M_{1}+M_{2}}\right|} \mathbf{1}\right)(x) .
$$


First, since $\Pi^{k_{g a p}} \mathbf{P}_{s_{M_{1}+1}}^{\left|J_{M_{1}+1}\right|} \ldots \mathbf{P}_{s_{M_{1}+M_{2}}}^{\left|J_{M_{1}+M_{2}}\right|} \mathbf{1}=\nu\left(\mathbf{P}_{s_{M_{1}+1}}^{\left|J_{M_{1}+1}\right|} \ldots \mathbf{P}_{s_{M_{1}+M_{2}}}^{\left|J_{M_{1}+M_{2}}\right|} \mathbf{1}\right) \mathbf{1}$, we may write, setting $\psi_{2}(s):=\nu\left(\mathbf{P}_{s_{M_{1}+1}}^{\left|J_{M_{1}+1}\right|} \ldots \mathbf{P}_{s_{M_{1}+M_{2}}}^{\left|J_{M_{1}+M_{2}}\right|} \mathbf{1}\right)$

$$
\begin{aligned}
\phi_{\Pi}(t, s) & =\psi_{2}(s)\left(\mathbf{P}^{j_{0}} \mathbf{P}_{t_{1}}^{\left|J_{1}\right|} \ldots \mathbf{P}_{t_{M_{1}}}^{\left|J_{M_{1}}\right|} \mathbf{1}\right)(x) \\
& =\psi_{2}(s) \phi_{1}(t) .
\end{aligned}
$$

Notice that $\phi_{2}(s)=\left(\mathbf{P}^{k_{g a p}+j_{M_{1}}} \mathbf{P}_{s_{M_{1}+1}}^{\left|J_{M_{1}+1}\right|} \ldots \mathbf{P}_{s_{M_{1}+M_{2}}}^{\left|J_{M_{1}+M_{2}}\right|} \mathbf{1}\right)(x)$; using the equality $\nu \mathbf{P}=\nu$, one gets $\psi_{2}(s)=\nu\left(\mathbf{P}^{k_{g a p}+j_{M_{1}}} \mathbf{P}_{s_{M_{1}+1}}^{\left|J_{M_{1}+1}\right|} \ldots \mathbf{P}_{s_{M_{1}+M_{2}}}^{\left|J_{M_{1}+M_{2}}\right|} \mathbf{1}\right)$ which allows us to control the difference between $\psi_{2}$ and $\phi_{2}$, namely

$$
\begin{aligned}
\psi_{2}(s)-\phi_{2}(s)= & \left(\nu-\delta_{x}\right)\left(\mathbf{P}^{k_{g a p}+j_{M_{1}}} \mathbf{P}_{s_{M_{1}+1}}^{\left|J_{M_{1}+1}\right|} \ldots \mathbf{P}_{s_{M_{1}+M_{2}}}^{\left|J_{M_{1}+M_{2}}\right|} \mathbf{1}\right) \\
= & \left(\nu-\delta_{x}\right)\left(\Pi \mathbf{P}_{s_{M_{1}+1}}^{\left|J_{M_{1}+1}\right|} \ldots \mathbf{P}_{s_{M_{1}+M_{2}}}^{\left|J_{M_{1}+M_{2}}\right|} \mathbf{1}\right) \\
& +\left(\nu-\delta_{x}\right)\left(Q^{k_{g a p}} \mathbf{P}^{j_{M_{1}}} \mathbf{P}_{s_{M_{1}+1}}^{\left|J_{M_{1}+1}\right|} \ldots \mathbf{P}_{s_{M_{1}+M_{2}}}^{\left|J_{M_{1}+M_{2}}\right|} \mathbf{1}\right) \\
= & \left(\nu-\delta_{x}\right)(\mathbf{1}) \nu\left(\mathbf{P}_{s_{M_{1}+1}}^{\left|J_{M_{1}+1}\right|} \ldots \mathbf{P}_{s_{M_{1}+M_{2}}}^{\left|J_{M_{1}+M_{2}}\right|} \mathbf{1}\right) \\
& +\left(\nu-\delta_{x}\right)\left(Q^{k_{g a p}} \mathbf{P}^{k_{M_{1}}} \mathbf{P}_{s_{M_{1}+1}}^{\left|J_{M_{1}+1}\right|} \ldots \mathbf{P}_{s_{M_{1}+M_{2}}}^{J_{M_{1}+M_{2}} \mid} \mathbf{1}\right)
\end{aligned}
$$

with $\left(\nu-\delta_{x}\right)(\mathbf{1})=0 ;$ consequently

$$
\begin{aligned}
\left|\psi_{2}(s)-\phi_{2}(s)\right| & =\left|\left(\nu-\delta_{x}\right)\left(\left.Q^{k_{g a p}+j_{M_{1}}} \mathbf{P}_{s_{M_{1}+1}}\right|_{J_{M_{1}+1}} \ldots \mathbf{P}_{s_{M_{1}+M_{2}}}^{\left|J_{M_{1}+M_{2}}\right|} \mathbf{1}\right)\right| \\
& \leq C_{Q} C_{\mathbf{P}}^{1+M_{2}} \kappa^{k_{g a p}}\left(\|\nu\|_{\mathcal{B}^{\prime}}+\left\|\delta_{x}\right\|_{\mathcal{B}^{\prime}}\right)\|\mathbf{1}\|_{\mathcal{B}} .
\end{aligned}
$$

On the other hand, one easily gets

$$
\left|\phi_{Q}(t, s)\right| \leq C_{Q} C_{\mathbf{P}}^{1+M_{1}+M_{2}} \kappa^{k_{\text {gap }}}\|\mathbf{1}\|_{\mathcal{B}}\left\|\delta_{x}\right\|_{\mathcal{B}^{\prime}} .
$$

Writing $\phi(t, s)=\phi_{1}(t) \phi_{2}(s)+\phi_{1}(t)\left(\psi_{2}(s)-\phi_{2}(s)\right)+\phi_{Q}(t, s)$ and using the previous overestimations, one finally gets

$$
\left|\phi(t, s)-\phi_{1}(t) \phi_{2}(s)\right| \leq 2 C_{Q} C_{\mathbf{P}}^{1+M_{1}+M_{2}}\left(\|\nu\|_{\mathcal{B}^{\prime}}+\left\|\delta_{x}\right\|_{\mathcal{B}^{\prime}}\right) \kappa^{k_{g a p}}\|\mathbf{1}\|_{\mathcal{B}} .
$$

Lemma is proved. 
To prove Proposition 1 , set $k_{0}=\max \left\{1, \log _{2} C_{\mathbf{P}}\right\}$ so that $C_{\mathbf{P}} \leq 2^{k_{0}}$. Since $\max _{m=1, \ldots, M_{1}+M_{2}} \operatorname{card}\left(J_{m}\right) \geq 1$, one gets

$$
C_{\mathbf{P}}^{M_{1}+M_{2}} \leq 2^{k_{0}\left(M_{1}+M_{2}\right)} \leq\left(1+\max _{m=1, \ldots, M_{1}+M_{2}} \operatorname{card}\left(J_{m}\right)\right)^{k_{0}\left(M_{1}+M_{2}\right)} .
$$

Now, Proposition 1 follows from Lemma 10.

We now prove Proposition 2; we need two auxiliary lemmas.

Lemma 11. Assume that the Markov chain $\left(X_{n}\right)_{n \geq 0}$ and the function $f$ satisfy the hypotheses M1, M2, M3 and M4. Then, for any $l, k=0,1, \ldots$

$$
\left|\operatorname{Cov}_{\mathbb{P}_{x}}\left(f\left(X_{l}\right), f\left(X_{l+k}\right)\right)\right| \leq A(x) \kappa^{k \gamma / 4},
$$

for any positive constant $\gamma$ satisfying $0<\gamma \leq \min \{1,2 \delta\}$, where

$$
A(x)=c_{\delta}\left(1+C_{Q} C_{\mathbf{P}}^{2}\left(\|\nu\|_{\mathcal{B}^{\prime}}+\left\|\delta_{x}\right\|_{\mathcal{B}^{\prime}}\right)\|\mathbf{1}\|_{\mathcal{B}}+\mu_{\delta}^{2+\gamma}(x)\right)
$$

and $c_{\delta}$ is a constant depending only on $\delta$.

Proof. We give a proof involving Lemma 10. Let $V$ and $V^{\prime}$ be two independent identically distributed r.v.'s of mean 0, independent of $X_{l}$ and $X_{l+m}$ and whose common characteristic function is supported in the interval $\left[-\varepsilon_{0}, \varepsilon_{0}\right]$, for some $\varepsilon_{0}>0$. Set $Y_{l}=f\left(X_{l}\right)+V$ and $Y_{l+k}^{\prime}=f\left(X_{l+k}\right)+V^{\prime}$.

Let $\widetilde{\phi}_{1}$ be the characteristic function of $Y_{l}, \widetilde{\phi}_{2}$ be the characteristic function of $Y_{l+k}^{\prime}$ and $\widetilde{\phi}(t, u)$ be the characteristic function of the vector $\left(Y_{l}, Y_{l+k}^{\prime}\right)$. Set $g_{T}(x)=x 1_{(|x| \leq T)}$ and $h_{T}(x, y)=g_{T}(x) g_{T}(y)$ for $x, y \in \mathbb{R}$. Let $\widehat{g}_{T}$ (resp. $\widehat{h}_{T}$ ) be the Fourier transform of the function $g_{T}\left(\right.$ resp. $\left.h_{T}\right)$ defined by

$$
\widehat{g}_{T}(t)=\int e^{i t x} g_{T}(x) d x
$$

$\left(\operatorname{resp} . \widehat{h}_{T}(t, u)=\iint e^{i(t x+u y)} h_{T}(x, y) d x d y=\widehat{g}_{T}(t) \widehat{g}_{T}(u).\right)$

For any $T>0$ and $l \geq 1, k \geq 0$, one gets

$$
\mathbb{E}_{x} f\left(X_{l}\right) f\left(X_{l+k}\right)=\mathbb{E}_{x} Y_{l} Y_{l+k}^{\prime}=\mathbb{E}_{x} h_{T}\left(Y_{l}, Y_{l+k}^{\prime}\right)+R_{0}
$$

with

$$
R_{0} \leq \mathbb{E}_{x} Y_{l} Y_{l+k}^{\prime} 1_{\left(\left|Y_{l}\right|>T\right)}+\mathbb{E}_{x} Y_{l} Y_{l+k}^{\prime} 1_{\left(\left|Y_{l+k}^{\prime}\right|>T\right)}
$$

By the inverse Fourier transform, one may write

$$
\mathbb{E}_{x} f\left(X_{l}\right) f\left(X_{l+k}\right)=\frac{1}{(2 \pi)^{2}} \iint \overline{\widehat{h}_{T}(t, u)} \widetilde{\phi}(t, u) d t d u+R_{0} .
$$

Analogously

$$
\mathbb{E}_{x} f\left(X_{l}\right)=\mathbb{E}_{x} Y_{l}=\mathbb{E}_{x} g_{T}\left(Y_{l}\right)+R_{1}=\frac{1}{2 \pi} \int \overline{\widehat{g}_{T}(t)} \widetilde{\phi}_{1}(t) d t+R_{1}
$$


and

$$
\mathbb{E}_{x} f\left(X_{l+k}\right)=\mathbb{E}_{x} Y_{l+k}^{\prime}=\mathbb{E}_{x} g_{T}\left(Y_{l+k}^{\prime}\right)+R_{2}=\frac{1}{2 \pi} \int \overline{\widehat{g}_{T}(u)} \widetilde{\phi}_{2}(u) d u+R_{2},
$$

where

$$
R_{1}:=\mathbb{E}_{x} Y_{l} 1_{\left(\left|Y_{l}\right|>T\right)} \text { and } R_{2}:=\mathbb{E}_{x} Y_{l+k}^{\prime} 1_{\left(\left|Y_{l+k}^{\prime}\right|>T\right)}
$$

This gives

$\operatorname{Cov}_{\mathbb{P}_{x}}\left(f\left(X_{l}\right), f\left(X_{l+k}\right)\right)=\mathbb{E}_{x} f\left(X_{l}\right) f\left(X_{l+k}\right)-\mathbb{E}_{x} f\left(X_{l}\right) \mathbb{E}_{x} f\left(X_{l+k}\right)$

where

$$
R=R_{0}+R_{1} \mathbb{E}_{x} g_{T}\left(Y_{l+k}^{\prime}\right)+R_{2} \mathbb{E}_{x} g_{T}\left(Y_{l}\right)+R_{1} R_{2}
$$

Note that

$$
\left|\iint \widehat{\widehat{h}}_{T}(t, u)\left(\widetilde{\phi}(t, u)-\widetilde{\phi}_{1}(t) \widetilde{\phi}_{2}(u)\right) d t d u\right| \leq\left\|\widehat{h}_{T}\right\|_{L^{2}}\left\|\widetilde{\phi}-\widetilde{\phi}_{1} \widetilde{\phi}_{2}\right\|_{L^{2}},
$$

Since $V, V^{\prime}$ are independent of $X_{l}, X_{l+k}$, we have $\widetilde{\phi}(t, u)=\phi(t, u) \mathbb{E}_{x} e^{i t V} \mathbb{E}_{x} e^{i u V^{\prime}}$ and $\widetilde{\phi}_{1}(t)=$ $\phi_{1}(t) \mathbb{E}_{x} e^{i t V}, \widetilde{\phi}_{2}(u)=\phi_{2}(u) \mathbb{E}_{x} e^{i u V^{\prime}}$, where

$$
\phi(t, u):=\mathbb{E}_{x} e^{i t f\left(X_{l}\right)+i u f\left(X_{l+k}\right)}=\left(\mathbf{P}^{l-1} \mathbf{P}_{t} \mathbf{P}^{k-1} \mathbf{P}_{u} \mathbf{1}\right)(x)
$$

and $\phi_{1}(t)=\mathbb{E}_{x} e^{i t f\left(X_{l}\right)}=\left(\mathbf{P}^{l-1} \mathbf{P}_{t} \mathbf{1}\right)(x), \phi_{2}(u)=\mathbb{E}_{x} e^{i u f\left(X_{l+k}\right)}=\left(\mathbf{P}^{l-1} \mathbf{P}_{t} \mathbf{1}\right)(x)$. Since the support of the characteristic functions of $V$ and $V^{\prime}$ is the interval $\left[-\varepsilon_{0}, \varepsilon_{0}\right]$ the function $\widetilde{\phi}-\widetilde{\phi}_{1} \widetilde{\phi}_{2}$ vanishes outside the square $\left[-\varepsilon_{0}, \varepsilon_{0}\right]^{2}$. Then, by Lemma 10 ,

$$
\begin{aligned}
\left\|\widetilde{\phi}-\widetilde{\phi}_{1} \widetilde{\phi}_{2}\right\|_{L^{2}} & \leq 2 \varepsilon_{0} \sup _{|t| \leq \varepsilon_{0},|u| \leq \varepsilon_{0}}\left|\phi(t, u)-\phi_{1}(t) \phi_{2}(u)\right| \\
& \leq 4 \epsilon_{0} C_{Q} C_{\mathbf{P}}^{3} \kappa^{k}\left(\|\nu\|_{\mathcal{B}^{\prime}}+\left\|\delta_{x}\right\|_{\mathcal{B}^{\prime}}\right)\|\mathbf{1}\|_{\mathcal{B}} .
\end{aligned}
$$

Using the inequality

$$
\left\|\widehat{h}_{T}\right\|_{L^{2}}^{2}=\iint h_{T}^{2}(x, y) d x d y=\left(\int g_{T}^{2}(x) d x\right)^{2} \leq \frac{4}{9} T^{6},
$$

one obtains

$$
\left|\operatorname{Cov}_{\mathbb{P}_{x}}\left(f\left(X_{l}\right), f\left(X_{l+k}\right)\right)\right| \leq \frac{2}{3 \pi^{2}} T^{3} \epsilon_{0} C_{Q} C_{\mathbf{P}}^{3} \kappa^{k}\left(\|\nu\|_{\mathcal{B}^{\prime}}+\left\|\delta_{x}\right\|_{\mathcal{B}^{\prime}}\right)\|\mathbf{1}\|_{\mathcal{B}}+|R| .
$$

Now we shall give a bound for $|R|$. By Hölder's inequality, for some $q_{\delta} \in(0,1)$ satisfying $\frac{1}{1+\delta}+\frac{1}{q_{\delta}}=1$,

$$
\mathbb{E}_{x}\left|Y_{l}\right|\left|Y_{l+k}^{\prime}\right| 1_{\left(\left|Y_{l}\right|>T\right)} \leq\left(\mathbb{E}_{x}\left|Y_{l}\right|^{2+2 \delta}\right)^{\frac{1}{2+2 \delta}}\left(\mathbb{E}_{x}\left|Y_{l+k}\right|^{2+2 \delta}\right)^{\frac{1}{2+2 \delta}} \mathbb{P}_{x}\left(\left|Y_{l}\right|>T\right)^{\frac{1}{q_{\delta}}}
$$


Using hypothesis M4, we have

$$
\left(\mathbb{E}_{x}\left|Y_{l}\right|^{2+2 \delta}\right)^{\frac{1}{2+2 \delta}} \leq c_{\delta}\left(\left(\mathbb{E}_{x}\left|f\left(X_{l}\right)\right|^{2+2 \delta}\right)^{\frac{1}{2+2 \delta}}+\left(\mathbb{E}_{x}|V|^{2+2 \delta}\right)^{\frac{1}{2+2 \delta}}\right) \leq c_{\delta} A_{0}(x)
$$

with $A_{0}(x)=\mu_{\delta}(x)+c_{\delta}^{\prime}$. Similarly $\left(\mathbb{E}_{x}\left|Y_{l+k}^{\prime}\right|^{2+2 \delta}\right)^{\frac{1}{2+2 \delta}} \leq c_{\delta} A_{0}(x)$. On the other hand, for any $\gamma \in(0,2 \delta]$, one gets

$$
\mathbb{P}_{x}\left(\left|Y_{l}\right|>T\right) \leq \frac{1}{T^{\gamma q_{\delta}}} \mathbb{E}_{x}\left|Y_{l}\right|^{\gamma q_{\delta}} \leq \frac{c_{\delta}}{T^{\gamma q_{\delta}}} A_{0}^{\gamma q_{\delta}}(x)
$$

Putting together these bounds gives

$$
\mathbb{E}_{x}\left|Y_{l}\right|\left|Y_{l+k}^{\prime}\right| 1_{\left(\left|Y_{l}\right|>T\right)} \leq c_{\delta} T^{-\gamma} A_{0}^{2+\gamma}(x) .
$$

In the same way we obtain, for some $c_{\delta}>0$ and any $\gamma \in(0,2 \delta]$,

$$
\mathbb{E}_{x}\left|Y_{l}\right|\left|Y_{l+k}^{\prime}\right| 1\left(\left|Y_{l+k}^{\prime}\right|>T\right) \leq c_{\delta} T^{-\gamma} A_{0}^{2+\gamma}(x) .
$$

From (9.7), (9.15), (9.16), it follows that

$$
\left|R_{0}\right| \leq c_{\delta} T^{-\gamma} A_{0}^{2+\gamma}(x) .
$$

From (9.16), taking $k=0$ we get, for some $c_{\delta}>0$ and any $\gamma \in(0,2 \delta]$,

$$
\max \left\{R_{1}, R_{2}\right\} \leq \sup _{l \geq 0}\left(\mathbb{E}_{x} Y_{l}^{2} 1\left(\left|Y_{l}\right|>T\right)\right)^{\frac{1}{2}} \leq c_{\delta} T^{-\gamma / 2} A_{0}^{1+\gamma / 2}(x)
$$

Since

$$
\left|\mathbb{E}_{x} g_{T}\left(Y_{l}\right)\right| \leq\left(\mathbb{E}_{x}\left(\left|Y_{l}\right|^{2+2 \delta}\right)\right)^{\frac{1}{2+2 \delta}} \leq c_{\delta} A_{0}(x)
$$

and

$$
\left|\mathbb{E}_{x} g_{T}\left(Y_{l+k}^{\prime}\right)\right| \leq c_{\delta} A_{0}(x),
$$

from (9.17), (9.18), it follows that, for some $c_{\delta}>0$ and any $\gamma \in(0,2 \delta]$, assuming without loss of generalities that $A_{0}(x) \geq 1$

$$
|R| \leq c_{\delta} T^{-\gamma / 2} A_{0}^{2+\gamma}(x) .
$$

The inequalities (9.14) and (9.19) yield, for some $c_{\delta}>0$ and any $\gamma \in(0,2 \delta]$,

$$
\begin{aligned}
\left|\operatorname{Cov}_{\mathbb{P}_{x}}\left(f\left(X_{l}\right), f\left(X_{l+k}\right)\right)\right| \leq & \frac{2}{3 \pi^{2}} T^{3} \varepsilon_{0} C_{Q} C_{\mathbf{P}}^{3} \kappa^{k}\left(\|\nu\|_{\mathcal{B}^{\prime}}+\left\|\boldsymbol{\delta}_{x}\right\|_{\mathcal{B}^{\prime}}\right)\|e\|_{\mathcal{B}} \\
& +c_{\delta}^{\prime} T^{-\gamma / 2} A_{0}^{2+\gamma}(x) .
\end{aligned}
$$

Choosing $T=\kappa^{-k / 4}$, it follows that

$$
\left|\operatorname{Cov}_{\mathbb{P}_{x}}\left(f\left(X_{l}\right), f\left(X_{l+k}\right)\right)\right| \leq A(x) c_{\delta} \kappa^{k \min \{1, \gamma / 2\} / 4},
$$

which proves the assertion of the lemma. 
Lemma 12. Assume that the Markov chain $\left(X_{n}\right)_{n \geq 0}$ and the function $f$ satisfy the hypotheses M1, M2, M3 and M4. Let $0<\gamma \leq \min \{1,2 \delta\}$. Then: that

a) There exists a sequence of (possibly complex) numbers $\left(s_{k}\right)_{k \geq 0}$ not depending on $x$ such

$$
\left|\operatorname{Cov}_{\mathbb{P}_{x}}\left(f\left(X_{l}\right), f\left(X_{l+k}\right)\right)-s_{k}\right| \leq A_{2}(x) \kappa^{l \gamma / 4-1}
$$

where

$$
\begin{aligned}
A_{2}(x)= & c_{\delta}\left(1+\mu_{\delta}(x)^{2+\gamma}\right. \\
& \left.+\left\|\boldsymbol{\delta}_{x}\right\|_{\mathcal{B}^{\prime}}\|e\|_{\mathcal{B}}\left(C_{\mathbf{P}}^{2} C_{Q}\left(\|\nu\|_{\mathcal{B}^{\prime}}\|e\|_{\mathcal{B}}+C_{Q}\right)+C_{\mathbf{P}} C_{Q}\left(1+\|\nu\|_{\mathcal{B}^{\prime}} C_{\mathbf{P}}\right)\right)\right)
\end{aligned}
$$

and the constant $c_{\delta}$ is depending only on $\delta$. Moreover, for $k \geq 0$,

$$
\left|s_{k}\right| \leq A_{2}(x) \kappa^{k \gamma / 4-1}
$$

and

$$
\left|s_{0}\right|+2 \sum_{k=1}^{\infty}\left|s_{k}\right| \leq A_{2}(x) c_{\gamma, \kappa}
$$

where $c_{\gamma, \kappa}$ is a constant depending only on its indices.

b) There exists a real number $\mu$ not depending on $x$ such that, for any $k \geq 1$,

$$
\left|\mathbb{E}_{x} f\left(X_{k}\right)-\mu\right| \leq A_{1}(x) \kappa^{k \gamma / 4-1}
$$

where $A_{1}(x)=c\left\|\boldsymbol{\delta}_{x}\right\|_{\mathcal{B}^{\prime}}\|e\|_{\mathcal{B}} C_{\mathbf{P}} C_{Q}+c_{\delta} \mu_{\delta}(x)^{1+\gamma}$. Moreover

$$
\sum_{k=0}^{\infty}\left|\mathbb{E}_{x} f\left(X_{k}\right)-\mu\right| \leq \bar{\mu}(x)=A_{1}(x) c_{\gamma, \kappa}
$$

where $c_{\gamma, \kappa}$ is a constant depending only on its indices.

Proof. We shall prove the part a). We keep the notations from the proof of Lemma 11. Denote $\widetilde{\phi}_{0}(t, u)=\widetilde{\phi}(t, u)-\widetilde{\phi}_{1}(t) \widetilde{\phi}_{2}(u)$. By (9.10), for any $l=0,1, \ldots$

$$
\operatorname{Cov}_{\mathbb{P}_{x}}\left(f\left(X_{l}\right), f\left(X_{l+k}\right)\right)=\frac{1}{(2 \pi)^{2}} \iint \overline{\widehat{h}_{T}(t, u)} \widetilde{\phi}_{0}(t, u) d t d u+R,
$$

with $R$ defined by (9.11). Since $V, V^{\prime}$ are independent of $X_{l}, X_{l+k}$,

$$
\begin{aligned}
\widetilde{\phi}(t, u) & =\left(\mathbb{E}_{x} e^{i 0 \sum_{j=1}^{l-1} X_{j}+i t X_{l}+i 0 \sum_{j=l+1}^{l+k-1} X_{j}+i u X_{l+k}}\right) \mathbb{E}_{x} e^{i t V} \mathbb{E}_{x} e^{i u V^{\prime}} \\
& =\left(\mathbf{P}^{l-1} \mathbf{P}_{t} \mathbf{P}^{k-1} \mathbf{P}_{u} e\right)(x) \mathbb{E}_{x} e^{i t V} \mathbb{E}_{x} e^{i u V^{\prime}} .
\end{aligned}
$$

Note that, for $k, l \geq 2$,

$$
\begin{aligned}
\left(\mathbf{P}^{l-1} \mathbf{P}_{t} \mathbf{P}^{k-1} \mathbf{P}_{u} e\right)(x)= & \boldsymbol{\delta}_{x}\left(\mathbf{P}^{l-1} \mathbf{P}_{t} \mathbf{P}^{k-1} \mathbf{P}_{u} e\right) \\
= & \boldsymbol{\delta}_{x}\left(\Pi \mathbf{P}_{t} \mathbf{P}^{k-1} \mathbf{P}_{u} e\right)+\boldsymbol{\delta}_{x}\left(Q^{l-1} \mathbf{P}_{t} \mathbf{P}^{k-1} \mathbf{P}_{u} e\right) \\
= & \nu\left(\mathbf{P}_{t} \mathbf{P}^{k-1} \mathbf{P}_{u} e\right) \\
& +\boldsymbol{\delta}_{x}\left(Q^{l-1} \mathbf{P}_{t} \Pi \mathbf{P}_{u} e\right)+\nu\left(Q^{l-1} \mathbf{P}_{t} Q^{k-1} \mathbf{P}_{u} e\right)
\end{aligned}
$$

Since

$$
\left|\boldsymbol{\delta}_{x}\left(Q^{l-1} \mathbf{P}_{t} \Pi \mathbf{P}_{u} e\right)\right|=\left|\boldsymbol{\delta}_{x}\left(Q^{l-1} \mathbf{P}_{t} e\right) \nu\left(\mathbf{P}_{u} e\right)\right| \leq \kappa^{l-1} C_{Q} C_{\mathbf{P}}^{2}\left\|\boldsymbol{\delta}_{x}\right\|_{\mathcal{B}^{\prime}}\|\nu\|_{\mathcal{B}^{\prime}}\|e\|_{\mathcal{B}}^{2}
$$


and

we obtain

$$
\left|\boldsymbol{\delta}_{x}\left(Q^{l-1} \mathbf{P}_{t} Q^{k-1} \mathbf{P}_{u} e\right)\right| \leq \kappa^{l+k-2} C_{Q}^{2} C_{\mathbf{P}}^{2}\left\|\boldsymbol{\delta}_{x}\right\|_{\mathcal{B}^{\prime}}\|e\|_{\mathcal{B}^{\prime}},
$$

$$
|\widetilde{\phi}(t, u)-\widetilde{\psi}(t, u ; k)| \leq \kappa^{l-1} C_{\mathbf{P}}^{2} C_{Q}\left(\|\nu\|_{\mathcal{B}^{\prime}}\|e\|_{\mathcal{B}}+C_{Q}\right)\left\|\boldsymbol{\delta}_{x}\right\|_{\mathcal{B}^{\prime}}\|e\|_{\mathcal{B}^{\prime}}
$$

where

$$
\widetilde{\psi}(t, u ; k)=\nu\left(\mathbf{P}_{t} \mathbf{P}^{k-1} \mathbf{P}_{u} e\right) \mathbb{E}_{x} e^{i t V} \mathbb{E}_{x} e^{i u V^{\prime}} .
$$

Note that $\widetilde{\psi}(t, u ; k)$ does not depend on the initial state $x$ since $V$ and $V^{\prime}$ are independent of the Markov chain. In the same way

$$
\begin{aligned}
\widetilde{\phi}_{1}(t) & =\left(\mathbf{P}^{l-1} \mathbf{P}_{t} e\right)(x) \mathbb{E}_{x} e^{i t V} \\
\widetilde{\phi}_{2}(u) & =\left(\mathbf{P}^{l+k-1} \mathbf{P}_{u} e\right)(x) \mathbb{E}_{x} e^{i u V^{\prime}},
\end{aligned}
$$

where, for $m \geq 2$,

$$
\begin{aligned}
\left(\mathbf{P}^{m-1} \mathbf{P}_{t} e\right)(x) & =\boldsymbol{\delta}_{x}\left(\mathbf{P}^{m-1} \mathbf{P}_{t} e\right) \\
& =\boldsymbol{\delta}_{x}\left(\Pi \mathbf{P}_{t} e\right)+\boldsymbol{\delta}_{x}\left(Q^{m-1} \mathbf{P}_{t} e\right) \\
& =\nu\left(\mathbf{P}_{t} e\right)+\boldsymbol{\delta}_{x}\left(Q^{m-1} \mathbf{P}_{t} e\right) .
\end{aligned}
$$

Since $\left|\boldsymbol{\delta}_{x}\left(Q^{m-1} \mathbf{P}_{t} e\right)\right| \leq \kappa^{m-1}\left\|\boldsymbol{\delta}_{x}\right\|_{\mathcal{B}^{\prime}}\|e\|_{\mathcal{B}} C_{\mathbf{P}} C_{Q}$, we get

$$
\begin{aligned}
\left|\widetilde{\phi}_{1}(t)-\widetilde{\psi}_{1}(t)\right| & \leq \kappa^{l-1}\left\|\boldsymbol{\delta}_{x}\right\|_{\mathcal{B}^{\prime}}\|e\|_{\mathcal{B}} C_{\mathbf{P}} C_{Q}, \\
\left|\widetilde{\phi}_{2}(u)-\widetilde{\psi}_{1}(u)\right| & \leq \kappa^{l+k-1}\left\|\boldsymbol{\delta}_{x}\right\|_{\mathcal{B}^{\prime}}\|e\|_{\mathcal{B}} C_{\mathbf{P}} C_{Q},
\end{aligned}
$$

where

$$
\widetilde{\psi}_{1}(t)=\nu\left(\mathbf{P}_{t} e\right) \mathbb{E}_{x} e^{i t V}=\nu\left(\mathbf{P}_{t} e\right) \mathbb{E}_{x} e^{i t V^{\prime}}
$$

does not depend on the initial state $x$ of the Markov chain. Denote $\widetilde{\psi}_{0}(t, u ; k)=\widetilde{\psi}(t, u ; k)-$ $\widetilde{\psi}_{1}(t) \widetilde{\psi}_{1}(u)$. From (9.23) and (9.24) it follows

$$
\begin{aligned}
& \left|\widetilde{\phi}_{0}(t, u)-\widetilde{\psi}_{0}(t, u ; k)\right| \\
\leq & |\widetilde{\phi}(t, u)-\widetilde{\psi}(t, u ; k)|+\left|\widetilde{\phi}_{1}(t) \widetilde{\phi}_{2}(u)-\widetilde{\psi}_{1}(t) \widetilde{\psi}_{1}(u)\right| \\
\leq & |\widetilde{\phi}(t, u)-\widetilde{\psi}(t, u ; k)|+\left|\widetilde{\phi}_{1}(t)-\widetilde{\psi}_{1}(t)\right|+\left|\nu\left(\mathbf{P}_{t} e\right)\right|\left|\left(\widetilde{\phi}_{2}(u)-\widetilde{\psi}_{1}(u)\right)\right| \\
\leq & \kappa^{l-1}\left\|\boldsymbol{\delta}_{x}\right\|_{\mathcal{B}^{\prime}}\|e\|_{\mathcal{B}} C_{\mathbf{P}}^{2} C_{Q}\left(\|\nu\|_{\mathcal{B}^{\prime}}\|e\|_{\mathcal{B}}+C_{Q}\right)+\kappa^{l-1}\left\|\boldsymbol{\delta}_{x}\right\|_{\mathcal{B}^{\prime}} C_{\mathbf{P}} C_{Q}\left(1+\left|\nu\left(\mathbf{P}_{t} e\right)\right|\right) \\
\leq & \kappa^{l-1}\left\|\boldsymbol{\delta}_{x}\right\|_{\mathcal{B}^{\prime}}\|e\|_{\mathcal{B}}\left(C_{\mathbf{P}}^{2} C_{Q}\left(\|\nu\|_{\mathcal{B}^{\prime}}\|e\|_{\mathcal{B}}+C_{Q}\right)+C_{\mathbf{P}} C_{Q}\left(1+\|\nu\|_{\mathcal{B}^{\prime}} C_{\mathbf{P}}\right)\right) \\
(9.26) \leq & C(x) \kappa^{l-1},
\end{aligned}
$$

where $C(x)=\left\|\boldsymbol{\delta}_{x}\right\|_{\mathcal{B}^{\prime}}\|e\|_{\mathcal{B}}\left(C_{\mathbf{P}}^{2} C_{Q}\left(\|\nu\|_{\mathcal{B}^{\prime}}\|e\|_{\mathcal{B}}+C_{Q}\right)+C_{\mathbf{P}} C_{Q}\left(1+\|\nu\|_{\mathcal{B}^{\prime}} C_{\mathbf{P}}\right)\right)$. Denote by $s_{k, T}$ the complex number defined by

$$
\left.s_{k, T}=\frac{1}{(2 \pi)^{2}} \iint \overline{\widehat{h}_{T}(t, u)} \widetilde{\psi}_{0}(t, u ; k) d t d u\right) .
$$


Note that $s_{k, T}$ does not depend on the initial state $x$ of the Markov chain since so is $\widetilde{\psi}_{0}(t, u ; k)$. With this notation we have

$$
\operatorname{Cov}_{\mathbb{P}_{x}}\left(f\left(X_{l}\right), f\left(X_{l+k}\right)\right)-s_{k, T}=R^{\prime}+R,
$$

where

$$
R^{\prime}=\frac{1}{(2 \pi)^{2}} \iint \overline{\widehat{h}_{T}(t, u)}\left(\widetilde{\phi}_{0}(t, u)-\widetilde{\psi}_{0}(t, u ; k)\right) d t d u .
$$

Since $\mathbb{E}_{x} e^{i t V} \mathbb{E}_{x} e^{i u V^{\prime}}$ has a support in the square $\left[-\varepsilon_{0}, \varepsilon_{0}\right]^{2}$, using (9.13) and (9.26) it follows that

$$
\left|R^{\prime}\right| \leq \frac{1}{(2 \pi)^{2}}\left\|\widehat{h}_{T}\right\|_{L^{2}}\left\|\widetilde{\phi}_{0}-\widetilde{\psi}_{0}\right\|_{L^{2}} \leq \frac{T^{3}}{3 \pi^{2}} \varepsilon_{0}^{2} C(x) \kappa^{l-1} .
$$

From (9.27) and (9.19), for some $c_{\delta}>0$, any $\gamma \in(0,2 \delta]$ and any $l, k=0,1, \ldots$,

$$
\begin{aligned}
\left|\operatorname{Cov}_{\mathbb{P}_{x}}\left(f\left(X_{l}\right), f\left(X_{l+k}\right)\right)-s_{k, T}\right| \leq & C(x) \frac{T^{3}}{3 \pi^{2}} \varepsilon_{0}^{2} \kappa^{l-1} \\
& +c_{\delta} T^{-\gamma} A_{0}^{2+\gamma}(x),
\end{aligned}
$$

From (9.28), for any $l, l^{\prime}=2,3, \ldots$ one obtains

$$
\begin{aligned}
& \left|\operatorname{Cov}_{\mathbb{P}_{x}}\left(f\left(X_{l}\right), f\left(X_{l+k}\right)\right)-\operatorname{Cov}_{\mathbb{P}_{x}}\left(f\left(X_{l^{\prime}}\right), f\left(X_{l^{\prime}+k}\right)\right)\right| \\
\leq & c_{\delta} T^{-\gamma} A_{0}(x)^{2+\gamma}+C(x) \frac{2 T^{3}}{3 \pi^{2}} \varepsilon_{0}^{2} \kappa^{\min \left\{l, l^{\prime}\right\}-1} .
\end{aligned}
$$

Taking $T=\kappa^{-\frac{1}{4} \min \left\{l, l^{\prime}\right\}}$ we get, for any $\gamma \leq \min \{1,2 \delta\}$,

$$
\mid \operatorname{Cov}_{\mathbb{P}_{x}}\left(f\left(X_{l}\right), f\left(X_{l+k}\right)-\operatorname{Cov}_{\mathbb{P}_{x}}\left(f\left(X_{l^{\prime}}\right), f\left(X_{l^{\prime}+k}\right) \mid \leq A(x) c_{\delta} \kappa^{\min \left\{l, l^{\prime}\right\} \gamma / 4-1},\right.\right.
$$

where $A(x)=c_{\delta}^{\prime}\left(A_{0}^{2+\gamma}(x)+C(x)\right)$, which proves that the sequence $\operatorname{Cov}_{\mathbb{P}_{x}}\left(f\left(X_{l}\right), f\left(X_{l+k}\right)\right.$, $l=1,2, \ldots$ is Cauchy. Denote by $s_{k}(x)$ its limit as $l \rightarrow \infty$. Taking the limit as $l \rightarrow \infty$ in (9.28), we get that

$$
\left|s_{k}(x)-s_{k, T}\right| \leq C(x) \frac{T^{3}}{3 \pi^{2}} \varepsilon_{0}^{2} \kappa^{l-1}+c_{\delta} T^{-\gamma} A_{0}^{2+\gamma}(x) .
$$

Letting $T=T_{l}=\kappa^{-\frac{l}{4}}$ this implies that $\lim _{l \rightarrow \infty} s_{k, T_{l}}=s_{k}(x)$. Since $s_{k, T_{l}}$ does not depend on $x$, we conclude that $s_{k}(x)$ is a constant $s_{k}$ which does not depend on $x$. Taking the limit as $l^{\prime} \rightarrow \infty$ in (9.29) we obtain (9.20).

The second assertion of the part a) of the lemma follows from (9.20) and Lemma 11 setting $l=k$.

The third assertion of the part a) follows immediately from the second one.

Let us now prove the part b). From (9.8), we have

$$
\left|\mathbb{E}_{x} f\left(X_{l}\right)-m_{T}\right| \leq \frac{1}{2 \pi} \int\left|\widehat{\widehat{g}}_{T}(t)\right|\left|\widetilde{\phi}_{1}(t)-\widetilde{\psi}_{1}(t)\right| d t+\left|R_{1}\right|
$$

where

$$
m_{T}=\frac{1}{2 \pi} \int \overline{\widehat{g}_{T}(t)} \widetilde{\psi}_{1}(t) d t
$$


$R_{1}$ is defined by (9.9) and $\widetilde{\psi}_{1}$ is defined by (9.25). Note that $m_{T}$ does not depend on $x$ since so is $\widetilde{\psi}(t)$. Taking into account the bounds in (9.18) and (9.23), we get

$$
\left|\mathbb{E}_{x} f\left(X_{l}\right)-m_{T}\right| \leq \kappa^{l-1}\left\|\boldsymbol{\delta}_{x}\right\|_{\mathcal{B}^{\prime}}\|e\|_{\mathcal{B}} C_{\mathbf{P}} C_{Q} \frac{1}{2 \pi} \int\left|\overline{\widehat{g}_{T}(t)}\right| d t+c_{\delta} T^{-\gamma} A_{0}^{1+\gamma}(x),
$$

where $A_{0}(x)=\mu_{\delta}(x)+c_{\delta}^{\prime}$. Recalling that $g_{T}(x)=x 1(|x| \leq T)$, to bound $\int\left|\overline{\widehat{g}_{T}(t)}\right| d t$ we use the usual isometry relation

$$
\left(\int\left|\overline{\widehat{g}_{T}(t)}\right| d t\right)^{2} \leq \int\left|\overline{\widehat{g}_{T}(t)}\right|^{2} d t=\int g_{T}^{2}(x) d x=\frac{2}{3} T^{3} .
$$

This implies, for any $\gamma \leq \min \{1,2 \delta\}$,

$$
\left|\mathbb{E}_{x} f\left(X_{l}\right)-m_{T}\right| \leq c\left\|\boldsymbol{\delta}_{x}\right\|_{\mathcal{B}^{\prime}}\|e\|_{\mathcal{B}} C_{\mathbf{P}} C_{Q} T^{3} \kappa^{l-1}+c_{\delta} T^{-\gamma} A_{0}^{1+\gamma}(x) .
$$

Taking $T=\kappa^{-\frac{l}{4}}$, we have

$$
\left|\mathbb{E}_{x} f\left(X_{l}\right)-m_{T}\right| \leq A_{1}(x) \kappa^{l \gamma / 4-1},
$$

where $A_{1}(x)=c\left\|\boldsymbol{\delta}_{x}\right\|_{\mathcal{B}^{\prime}}\|e\|_{\mathcal{B}} C_{\mathbf{P}} C_{Q}+c_{\delta} A_{0}^{1+\gamma}(x)$. From this inequality it follows that

$$
\left|\mathbb{E}_{x} f\left(X_{l}\right)-\mathbb{E}_{x} f\left(X_{k}\right)\right| \leq 2 A_{1}(x) \kappa^{\min \{l, k\} \gamma / 4-1},
$$

which proves that the sequence $\left(\mathbb{E}_{x} f\left(X_{l}\right)\right)_{l>1}$ is Cauchy and therefore has a limit denoted $\mu(x)$. Since $m_{T}$ does not depend on $x$, taking $\lim$ as $l \rightarrow \infty$ in (9.31) we conclude that $\mu(x)=\mu$ not depending on $x$. Taking $\lim _{k \rightarrow \infty}$ in (9.32), we get

$$
\left|\mathbb{E}_{x} f\left(X_{l}\right)-\mu\right| \leq 2 A_{1}(x) \kappa^{l \min \{1, \gamma\} / 4-1},
$$

which proves the first assertion of the part a) of the lemma. The second follows from the first one.

The part a) of the previous lemma shows that the bound 3.5 of Proposition 2 is satisfied. From the following lemma it follows that the bound 3.6 of Proposition 2 is also satisfied.

Lemma 13. Assume that the Markov chain $\left(X_{n}\right)_{n \geq 0}$ and the function $f$ satisfy the hypotheses M1, M2, M3 and M4. Let $0<\gamma \leq \min \{1,2 \bar{\delta}\}$. Then the series $s_{0}+2 \sum_{k=1}^{\infty}\left(s_{k}+s_{k}^{*}\right)$ converges to a non-negative number $\sigma^{2} \geq 0$, not depending on $x$, such that for any $n \geq 1$,

$$
\sup _{m \geq 1}\left|\operatorname{Var}_{\mathbb{P}_{x}}\left(\sum_{i=m}^{m+n-1} f\left(X_{i}\right)\right)-n \sigma^{2}\right| \leq \tau(x),
$$

where $\tau(x)=c_{\delta, \kappa, \gamma} A_{3}(x)$,

$$
A_{3}(x)=1+\mu_{\delta}(x)^{2+\gamma}+\left(1+\left\|\boldsymbol{\delta}_{x}\right\|_{\mathcal{B}^{\prime}}\right)\|e\|_{\mathcal{B}}\left(C_{\mathbf{P}}^{2} C_{Q}\left(1+C_{Q}\right)+C_{\mathbf{P}} C_{Q}\left(1+\|\nu\|_{\mathcal{B}^{\prime}} C_{\mathbf{P}}\right)\right)
$$

and $c_{\delta, \kappa, \gamma}$ is a constant depending only on the constants indicated in its indices.

Proof. First note that, from Lemma 12 and Lemma 11 we obtain, for $k=0,1, \ldots$,

$$
\mid \operatorname{Cov}_{\mathbb{P}_{x}}\left(f\left(X_{l}\right), f\left(X_{l+k}\right)-s_{k} \mid \leq A_{3}(x) c_{\delta, \kappa} \kappa^{c_{\gamma, \kappa} \max \{l, k\}},\right.
$$


for some constants $c_{\delta, \kappa}, c_{\gamma, \kappa}$ depending only on $\delta, \gamma$ and $\kappa$. Then, for any $k=0,1, \ldots$,

$$
\begin{aligned}
\sum_{l=m}^{m+n-1} \sum_{k=1}^{m+n-l} \mid \operatorname{Cov}_{\mathbb{P}_{x}}\left(f\left(X_{l}\right), f\left(X_{l+k}\right)-s_{k} \mid\right. & \leq A_{3}(x) c_{\delta, \kappa} \sum_{l=m}^{m+n-1} \sum_{k=1}^{m+n-l} e^{-c_{\gamma, \kappa} \max \{l, k\}} \\
& \leq A_{3}(x) c_{\delta, \gamma, \kappa}^{\prime \prime} .
\end{aligned}
$$

Since

$$
\operatorname{Var}_{\mathbb{P}_{x}}\left(\sum_{l=m}^{m+n-1} f\left(X_{l}\right)\right)=\sum_{l=m}^{m+n-1} \operatorname{Var}_{\mathbb{P}_{x}}\left(f\left(X_{l}\right)\right)+2 \sum_{l=m}^{m+n-1} \sum_{k=1}^{m+n-l} \operatorname{Cov}_{\mathbb{P}_{x}}\left(f\left(X_{l}\right), f\left(X_{l+k}\right)\right.
$$

we get

$$
\left|\operatorname{Var}_{\mathbb{P}_{x}}\left(\sum_{l=m}^{m+n-1} f\left(X_{l}\right)\right)-\left(n s_{0}+\sum_{l=m}^{m+n-1} \sum_{k=1}^{m+n-l}\left(s_{k}+s_{k}^{*}\right)\right)\right| \leq A_{3}(x) c_{\delta, \gamma, \kappa}^{\prime \prime} .
$$

Taking into account that, by Lemma $12,\left|s_{k}\right| \leq A_{2}(x) \kappa^{k \gamma / 4-1}$, we obtain

$$
\left|\operatorname{Var}_{\mathbb{P}_{x}}\left(\sum_{l=m}^{m+n-1} f\left(X_{l}\right)\right)-n\left(s_{0}+\sum_{k=1}^{\infty}\left(s_{k}+s_{k}^{*}\right)\right)\right| \leq A_{3}(x) c_{\delta, \gamma, \kappa}^{\prime \prime \prime},
$$

which proves (9.33).

It remains to show that the series $s_{0}+\sum_{k=1}^{\infty}\left(s_{k}+s_{k}^{*}\right)$ converges to a non-negative number $\sigma^{2} \geq 0$. Dividing by $n$ and taking the limit as $n \rightarrow \infty$ in (9.34), we deduce that $s_{0}+$ $\sum_{k=1}^{\infty}\left(s_{k}+s_{k}^{*}\right) \geq 0$.

9.2. Proof of Theorem 2. First note that conditions C1 and C3 are satisfied by Propositions 1 and 2. Condition C2 is satisfied by hypothesis M4. Let $\mu_{i}(x)=\mathbb{E}_{x} f\left(X_{i}\right)$. Let $\alpha<\delta$ and $\delta^{\prime}=\frac{1}{2}(\alpha+\delta)$. Since $\alpha<\delta^{\prime}$, from 1 with $\delta^{\prime}$ replacing $\delta$, it follows that for any $x \in \mathbb{X}$ there exists a probability space $\left(\Omega, \mathcal{F}, \mathbb{P}_{x}\right)$, a sequence of independent standard normal r.v.'s $\left(W_{i}^{\prime}\right)_{i \geq 1}$ and a sequence of r.v.'s $\left(Y_{i}^{\prime}\right)_{i \geq 1}$ such that $\left(Y_{i}^{\prime}\right)_{i \geq 1} \stackrel{d}{=}\left(f\left(X_{i}\right)\right)_{i \geq 1}$ and for any $0<\rho<\frac{1}{2} \frac{\alpha}{1+2 \alpha}$,

$$
\mathbb{P}_{x}\left(N^{-\frac{1}{2}} \sup _{k \leq N}\left|\sum_{i=1}^{k}\left(Y_{i}^{\prime}-\mu_{i}(x)-\sigma W_{i}^{\prime}\right)\right|>N^{-\rho}\right) \leq C_{0}(x) N^{-\alpha \frac{1+\alpha}{1+2 \alpha}+\rho(2+2 \alpha)},
$$

where $C_{0}(x)=C_{0}^{\prime}\left(1+\lambda_{0}(x)+\mu_{\delta^{\prime}}(x)+\sqrt{\tau(x)}\right)^{2+2 \delta^{\prime}}, C_{0}^{\prime}$ is a constant depending only on $\lambda_{1}, \lambda_{2}, \alpha, \delta, \sigma$ and $\lambda_{0}(x), \mu_{\delta}(x), \tau(x), \lambda_{1}, \lambda_{2}$ and $\sigma^{2}$ are defined in Propositions 1 and 2. If $\bar{\mu}(x) \leq N^{\frac{1}{2}-\rho}$ (with $\bar{\mu}(x)$ from Proposition 2 using (9.35) we have

$$
\begin{aligned}
& \mathbb{P}_{x}\left(N^{-\frac{1}{2}} \sup _{k \leq N}\left|\sum_{i=1}^{k}\left(Y_{i}^{\prime}-\mu-\sigma W_{i}^{\prime}\right)\right|>2 N^{-\rho}\right) \\
\leq & \mathbb{P}_{x}\left(\sup _{k \leq N}\left|\sum_{i=1}^{k}\left(Y_{i}^{\prime}-\mu_{i}(x)-\sigma W_{i}^{\prime}\right)\right|>2 N^{\frac{1}{2}-\rho}-\bar{\mu}(x)\right) \\
\leq & C_{0}(x) N^{-\alpha \frac{1+\alpha}{1+2 \alpha}+\rho(2+2 \alpha)} .
\end{aligned}
$$


If $\bar{\mu}(x)>N^{\frac{1}{2}-\rho}$, it is obvious that

$$
1 \leq\left(\bar{\mu}(x) N^{-\frac{1}{2}+\rho}\right)^{2 \alpha} \leq \bar{\mu}(x)^{2 \alpha} N^{-\alpha+2 \rho \alpha} .
$$

From (9.36) and (9.37) we get

$$
\begin{aligned}
& \mathbb{P}_{x}\left(N^{-\frac{1}{2}} \sup _{k \leq N}\left|\sum_{i=1}^{k}\left(Y_{i}^{\prime}-\mu-\sigma W_{i}^{\prime}\right)\right|>2 N^{-\rho}\right) \\
\leq & \left(C_{0}(x)+\bar{\mu}(x)^{2 \alpha}\right) N^{-\alpha \frac{1+\alpha}{1+2 \alpha}+\rho(2+2 \alpha)} .
\end{aligned}
$$

Taking into account the expressions for $\lambda_{0}(x), \mu_{\delta}(x), \tau(x), \lambda_{1}, \lambda_{2}, \bar{\mu}(x)$ and choosing $\gamma$ small we obtain

$$
C_{0}(x)+\bar{\mu}(x)^{2 \alpha} \leq C(x)=C_{1}\left(1+\left\|\boldsymbol{\delta}_{x}\right\|_{\mathcal{B}^{\prime}}+\mu_{\delta}(x)\right)^{2+2 \delta},
$$

where $C_{1}$ is a constant depending only on $\delta, \alpha, \kappa, C_{\mathbf{P}}, C_{Q},\|e\|_{\mathcal{B}},\|\nu\|_{\mathcal{B}^{\prime}}$.

Generally the measure $\mathbb{P}_{x}$ and the constructed sequence $\left(Y_{i}^{\prime}\right)_{i \geq 1}$ depend both on the initial state $x$. It is easy to reconstruct $\left(Y_{i}^{\prime}\right)_{i \geq 1}$ independently of $x$. Indeed, on the canonical space $\widetilde{\Omega}=\mathbb{R}^{\infty} \times \mathbb{R}^{\infty}$ there is a probability measure $\widetilde{\mathbb{P}}_{x}$ which coinside with the joint distribution of the sequence $\left(Y_{i}^{\prime}, W\right)_{i>1}$. It is enough to redefine $Y_{i}^{\prime}=\omega_{1, i}$ and $W_{i}=\omega_{2, i}$ as the coordinate processes, where $\omega=\left(\omega_{1}, \omega_{2}\right) \in \widetilde{\Omega}$. With this construction only the measure $\widetilde{\mathbb{P}}_{x}$ depends on the initial state $x$. The measurability of the map $x \in \mathbb{X} \rightarrow \widetilde{\mathbb{P}}_{x}(\cdot)$ follows from the construction.

9.3. Proof of Theorem 3. Assume the conditions of Theorem 2 and hypothesis M5. First we note that hypothesis $\mathbf{M} 5$ ensures the existence of the mean $\nu(f)=\mathbf{E}_{\nu} X_{k}=$ $\int\left(\mathbb{E}_{x} X_{k}\right) \nu(d x)$ and of the mixed moment $\mathbf{E}_{\nu}\left(X_{l} X_{l+k}\right)=\int \mathbb{E}_{x}\left(X_{l} X_{l+k}\right) \nu(d x)$ with respect to the invariant measure. By Proposition 2, we have $\lim _{k \rightarrow \infty} \mathbb{E}_{x} X_{k}=\mu, \nu$-a.s. on $\mathbb{X}$. Then by Lebesgue theorem on dominated convergence

$$
\nu(f)=\mathbf{E}_{\nu} X_{k}=\lim _{k \rightarrow \infty} \int\left(\mathbb{E}_{x} X_{k}\right) \nu(d x)=\int\left(\lim _{k \rightarrow \infty} \mathbb{E}_{x} X_{k}\right) \nu(d x)=\mu .
$$

Without loss of generality we can assume that $\nu(f)=0$. Using hypothesis M5 and $\nu(f)=0$, we have

$$
\begin{aligned}
\int \operatorname{Cov}_{\mathbf{P}_{x}}\left(X_{l}, X_{l+k}\right) \nu(d x) & =\int \mathbb{E}_{x}\left(X_{l} X_{l+k}\right) \nu(d x)-\int \mathbb{E}_{x}\left(X_{l}\right) \mathbb{E}_{x}\left(X_{l+k}\right) \nu(d x) \\
& =\mathbf{E}_{\nu}\left(X_{l} X_{l+k}\right)-\int \mathbb{E}_{x}\left(X_{l}\right) \mathbb{E}_{x}\left(X_{l+k}\right) \nu(d x) \\
& =\operatorname{Cov}_{\mathbb{P}_{\nu}}\left(X_{0}, X_{k}\right)-\int \mathbb{E}_{x}\left(X_{l}\right) \mathbb{E}_{x}\left(X_{l+k}\right) \nu(d x) .
\end{aligned}
$$

By Proposition 2, for any $x \in \mathbb{X}$, we have $\lim _{l \rightarrow \infty} \operatorname{Cov}_{\mathbf{P}_{x}}\left(X_{l}, X_{l+k}\right)=s_{k}$ and $\lim _{l \rightarrow \infty} \mathbb{E}_{x}\left(X_{l}\right)=$ 0 . As before, integrating with respect to the stationary measure and using Lebesgue theorem on dominated convergence, it follows that $s_{k}=\operatorname{Cov}_{\mathbb{P}_{\nu}}\left(X_{0}, X_{k}\right)$. Thus the conclusions of Theorem 2 hold true with $\mu=\nu(f)$ and $\sigma^{2}=\sigma_{\nu}^{2}$, which proves Theorem 3 . 


\section{REFERENCES}

[1] Berkes, I. and Philipp, W. (1979). Approximation Theorems for independent and weakly dependent random vectors. Ann. Probab. Vol. 7, 29-54. MR515811

[2] Bernstein, S. (1927) Sur l'extension du théorème limite du calcul des probabilités aux sommes de quantités dépendantes. Mathematische Annalen 97, 1-59.

[3] Borovkov, A.A. (1973). On the rate of convergence in the invariance principle. Probab. Theory and its Applications. XVIII, Vol. 2, p. 217-234.

[4] Billingsley, P. (1968). Convergence of Probability Measures. Willey, New York.

[5] Cuny, C. (2011). Pointwise ergodic theorems with rate and application to limit theorems for stationary processes. Stoch. Dyn. 11, no. 1, 135-155.

[6] Doukhan, P., Leon, J. R. and Portal, F. (1987). Principe d'invariance faible pour la mesure empirique d'une suite de variables aléatoires mélangeante. Probab. Th. Rel. Fields 76, 51-70.

[7] Dedecker, J., Doukhan, P. and Merlevède, F. (2012) Rates of convergence in the strong invariance principle under projective criteria. Electron. J. Probab. 17, no. 16, 1-31.

[8] Dudley, R. M. (1968). Distances of probability measures and random variables. Ann. Math. Statist. 39 1563-1572.

[9] Dunford, N. and Schwartz, J. T. (1958). Linear operators, Part I, Wiley-Interscience.

[10] Einmahl, U. (1989). Extensions of results of Komlós, Major and Tusnády to the multivariate case. J. Multivariate Anal. 28, 20-68. MR9968984

[11] Grama I. G. (1989). On the rate of convergence in the functional central limit theorem for semimartingales. Statistics and Control of Stochastic Processes. Proceedings of the Steklov seminar 1985-1986, Vol. 2, ed. by N.V.Krylov and A.N.Shiryaev. Optimization Software, INC. Publication Division. New York. Los Angeles, pp. 141-156.

[12] Grama, I. and Nussbaum, M. (1998) Asymptotic equivalence for nonparametric generalized linear model. Probab. Theory Relat. Fields 111, 167-214.

[13] Grama, I. and Nussbaum, M. (2002) Asymptotic equivalence for nonparametric regression. Mathematical methods of statistics 11, No 1, pp. 1-36.

[14] Grama, I. and Neumann. M. (2006) Asymptotic equivalence of nonparametric autoregression and nonparametric regression. Ann. of Statist. Vol. 34, No. 4, 1701-1732. DOI: 10.1214/009053606000000560

[15] Gouëzel, S. (2010). Almost sure invariance principle for dynamical systems by spectral methods. Ann. Prob. 38, 1639-1671. DOI: 10.1214/10-AOP525.

[16] Guivarc'h, Y. (1984). Application d'un théorème limite local à la transcience et à la récurrence de marches aléatoires. Lecture Notes in Math. Springer, 301-332.

[17] Guivarc'h, Y. and Le Page, E. (2008). On spectral properties of a family of transfer operators and convergence to stable laws for affine random walks. Ergod. Th. 83 Dynam. Sys. 28, 423-446. DOI $10.1017 / \mathrm{S} 0143385707001010$

[18] Guivarc'h, Y. and Le Page, E. (2004). Simplicity of the Lyapunov spectrum and spectral gap property for a family of transfer operators on projective space. Random walks and geometry, 181-259, Walter de Gruyter $\mathcal{E}^{3}$ Co. KG, Berlin.

[19] Hall, P. and Heyde, C. C. (1980). Martingale Limit Theory and its Application. Academic Press, New York.

[20] Hervé, L. and Pène, F. (2008). Nagaev method via Keller-Liverani theorem. Preprint.

[21] Keller, G. and Liverani, C. (1999). Stability of the spectrum for transfer operators. Ann. Sc. Norm. Super. Pisa Cl. Sci. (4) 28, 141-152. MR1679080

[22] Komlós, J., Major, P. and Tusnády, G. (1976). An approximation of partial sums of independent rv's and the sample df. II Z. Wahrsch. verw. Gebiete. 34, 33-58.

[23] Kubilius, K. (1994). Rate of convergence in the invariance principle for martingale difference arrays. Lithuanian Mathematical Journal 34, Number 4, 383-392, DOI: 10.1007/BF0233688

[24] Le Page E. (1982). Théorèmes limites pour les produits de matrices aléatoires. Springer Lecture Notes, 928 258-303. 
[25] Le Page E. (1983). Théorèmes de renouvellement pour les produits de matrices aléatoires. Equations aux différences aléatoires. Séminaires de Rennes.

[26] Le Page E. (1989). Régularité du plus grand exposant caractéristique des produits de matrices aléatoires indépendantes et applications. Ann. Inst. Henri Poincaré, Vol. 25, No 2, pp. 109-142.

[27] Liu, W. and Lin, Z. (2009). Strong approximation for a class of stationary processes. Stochastic Process. Appl. 119, 249-280. MR-2485027

[28] Merlevède, F. and Rio, E. (2012). Strong approximation of partial sums under dependence conditions with application to dynamical systems. Stochastic Process. Appl. 122, 386-417.

[29] Nagaev, S. V. (1957). Some limit theorems for stationary Markov chains. Theory of probability and its applications. 11 (4) 378-406.

[30] Nagaev, S. V. (1961) More exact statements of limit theorems for homogeneous Markov chains. Theory of probability and its applications. 6 (1), 62-81.

[31] Prokhorov, Ju. V. (1956). Convergence of random processes and limit theorems in probability. Theor. Probability Appl. 1, 157-214.

[32] Rio, E. (2000). Théorie asymptotique des processus aléatoires faiblement dépendants. Mathématiques et Applications 31, Springer-Verlag, Berlin, 2000. $\mathrm{x}+169$ pp. MR-2117923

[33] Rosenthal, H. P. (1970) On the subspaces of $L^{p}(p>2)$ spanned by sequences of independent random variables. Israel J. Math. 8, 273-303. MR0271721

[34] Sakhanenko, A. (1983). The rate of convergence in the invariance principle for non-identically distributed random variables with exponential moments. Limit theorems for sums of random variables. Trudy Inst. Matem., Acad. Nauk SSSR, Sibirsk. Otdel. (in Russian), Novosibirsk, Nauka, Vol. 3, 3-49.

[35] Sakhanenko, A. (1985). Estimates in the invariance principle. Trudy Inst. Matem., Acad. Nauk SSSR, Sibirsk. Otdel. (in Russian), Novosibirsk, Nauka, Vol. 5, 27-44.

[36] Sakhanenko, A. (1989). On the accuracy of normal approximation in the invariance principle. Trudy Inst. Matem., Acad. Nauk SSSR, Sibirsk. Otdel. (in Russian), Novosibirsk, Nauka, Vol. 19, 4-49.

[37] Sakhanenko, A.I. (2000). A new way to obtain estimates in the invariance principle. High dimensional probability, (II Seattle, WA, 1999), 223-245, Progr. Probab., 47, Birkäuser Boston, MA.

[38] Shao, Q.M. (1993). Almost sure invariance principles for mixing sequences of random variables. Stochastic Process. Appl. 48, 319-334. MR-1244549

[39] Serfling, R. J. (1970). Moments inequalities for the maximum cumulative sums. Ann. Math. Stat. Vol. 41, N. 4, 1227-1234. MR0268938

[40] Strassen, V. (1965). The existence of probability measures with given marginals. Ann. Math. Statist. $36423-439$.

[41] Wu, W. B. (2007) Strong invariance principles for dependent random variables. Ann. Probab. 35, 22942320. MR-2353389

[42] Zaitsev, A. Y. (1998). Multidimensional version of the results of Komlós, Major and Tusnády for vectors with finite exponential moments. ESAIM Probab. Stat. 2, 41-108. MR 1616527

[43] Zaitsev, A. Y. (2007). Estimates for the rate of strong approximation in the multidimensional invariance principle. J. Math. Sci. (N. Y.) 145 4856-4865. MR2355400

[44] Zhao, O. and Woodroofe, M. (2008). Law of the iterated logarithm for stationary processes. Ann. Probab. 36, 127-142. MR-2370600 
Current address, I. Grama: Université de Bretagne Sud, LMBA, Campus de Tohannic, BP 573, 56017 Vannes cedex, France

E-mail address: ion.grama@univ-ubs.fr

Current address, E. Le Page: Université de Bretagne Sud, LMBA, Campus de Tohannic, BP 57356017 Vannes cedex, France

E-mail address: emile.lepage@univ-ubs.fr

Current address, M. Peigné: Université F. Rabelais Tours, LMPT, Parc de Grandmont, 37200 Tours, France

E-mail address: peigne@lmpt.univ-tours.fr 